\title{
Weakly Enforced Essential Boundary Conditions for NURBS-embedded and trimmed NURBS geometries on the basis of the Finite Cell Method
}

\author{
M. Ruess ${ }^{1, *}$, D. Schillinger ${ }^{2}$, Y. Bazilevs ${ }^{3}$, V. Varduhn ${ }^{4}$, E. Rank ${ }^{4}$ \\ ${ }^{1}$ Aerospace Structures and Computational Mechanics, Delft University of Technology, Kluyverweg 1, 2629 HS \\ Delft, The Netherlands \\ ${ }^{2}$ Inst. for Computational Engineering and Sciences, University of Texas at Austin, 201 East 24th Street, Austin, \\ TX 78712, USA \\ ${ }^{3}$ Dept. of Structural Engineering, University of California, San Diego, 9500 Gilman Drive, La Jolla, CA 92093, \\ USA \\ ${ }^{4}$ Chair for Computation in Engineering, Technische Universität München, Arcisstraße 21, 80333 Munich, \\ Germany
}

\begin{abstract}
Enforcing essential boundary conditions plays a central role in immersed boundary methods. Nitsche's idea has proven to be a reliable concept to satisfy weakly boundary and interface constraints. We formulate an extension of Nitsche's method for elasticity problems in the framework of higher order and higher continuity approximation schemes such as the B-spline and NURBS-version of the finite cell method or isogeometric analysis on trimmed geometries. Furthermore, we illustrate a significant improvement of the flexibility and applicability of this extension in the modelling process of complex 3D geometries. With several benchmark problems we demonstrate the overall good convergence behavior of the proposed method and its good accuracy. We provide extensive studies on the stability of the method, its influence parameters and numerical properties, and a rearrangement of the numerical integration concept that in many cases reduces the numerical effort by a factor two. A newly composed boundary integration concept further enhances the modelling process and allows a flexible, discretization-independent introduction of boundary conditions. Finally, we present our strategy in the framework of the modelling and isogeometric analysis process of trimmed NURBS geometries.
\end{abstract}

Keywords: NURBS, weak boundary conditions, isogeometric analysis, trimmed geometry, fictitious domain, immersed boundary, high order approximation

\section{Introduction}

In contrast to standard finite elements, fictitious domain methods do not require a boundary-fitted mesh. Instead, they embed structures of an arbitrarily complex geometry in an analysis domain of simple shape, thus omitting the time-consuming process of mesh generation. The finite cell

${ }^{*}$ Corresponding author:

Delft University of Technology, Aerospace Structures and Computational Mechanics, Kluyverweg 1, 2629 HS Delft, The Netherlands, email: m.ruess@ @udelft.nl 
method (FCM) $[49,39]$ is a high-order approximation scheme that follows the fictitious domain idea. The fundamental concept of the method is independent of the applied approximation basis and has been successfully implemented and tested for integrated Legendre polynomials $[19,44]$ as well as for B-splines [49, 47] and NURBS [42], termed as the $p-$, B-spline and NURBS versions of the FCM, respectively. The $p-$ and B-spline versions denote implementations of the method that exploit the advantages of Cartesian grids, disregarding any shape information for the embedding domain. In contrast, the NURBS version refers to an embedding domain that is represented by exact CAD-based NURBS-geometries used for trimmed shapes in isogeometric analysis [16, 31, 9, 7]. All FCM versions use the fictitious domain concept to incorporate arbitrarily complex and filigree details in the simulation model. Like other embedded domain $[34,36]$ and immersed boundary methods $[35,40]$ the finite cell method does not necessarily require an explicit domain representation in terms of boundary fitted segments or elements but instead exploits recursive bisection [37] to regain control adaptively over the solution domain. Other domain representations include voxel models derived from CT-data [44, 55, 54] or surface models in combination with a kd-tree-based radiosity method [13] that performs the required inside/outside test for any point of the simulation domain [42]. However, due to the absence of boundary-fitted elements in all representations, the imposition of essential boundary conditions turns out to be a key challenge, which in many cases can greatly influence the accuracy of the analysis and limit the modelling process. A reliable and accurate strong imposition is uncomplicated only in cases where the boundary of the simulation domain fully coincides with the boundary of the solution domain. All other cases run the risk of loosing stability and accuracy due to, e.g., a displacement field that is constrained in too few or too many locations.

Following the weak formulation of the finite element method several efforts have been made over the years to satisfy essential boundary conditions in a weak sense as an alternative to equivalent pointwise constraints. When weak enforcement of boundary conditions is employed, no explicit constraints on the displacement field are introduced. Instead, the variational formulation of structural mechanics is modified to enforce displacement boundary conditions as EulerLagrange conditions. The most popular strategies include the straightforward, but variationally inconsistent, penalty method $[3,58]$. The method is insensitive with regard to linear dependence of the constraints and retains the positive definiteness of the governing system of equations. Unfortunately this simple approach may suffer significantly from the imbalance between accuracy and violation of the constraint conditions due to a free choice of the penalty value that dominates the conditioning of the governing equations with serious effects on the solution procedure [21]. The Lagrange Multiplier Method [2, 29, 15, 24] is in close relation to the penalty approach that, though being variationally consistent, introduces additional unknowns and destroys positive definiteness of the augmented system of equations. Previous work on weak enforcement of essential boundary conditions also includes the pioneering effort of NITSCHE [38] for the Poisson problem which has been successfully adapted to structural mechanics $[25,22,27,20]$, biomechanics [44], and fluid mechanics $[12,11]$. Besides being computationally convenient, weakly enforced boundary conditions show a significant increase in accuracy over their strongly imposed counterparts for wall-bounded turbulent flows [12].

In this framework, the focus of the present paper is on the application of the weak boundary conditions within the finite cell method. We demonstrate that weakly enforced boundary condi- 
tions can considerably enhance the flexibility of the finite cell method and that the combination of the two even provides a conceptual strategy to overcome the problem of trimmed geometries $[50,42]$ in the standard isogeometric analysis approach. We start by providing a detailed general derivation of the method in the framework of the FCM, unifying earlier work: In a first approach to enforce homogeneous boundary conditions in a weak sense, DÜSTER ET AL. [19] propose embedding the constraint boundary part in a very stiff material in terms of a finite cell penalization with a high penalty value in the extension domain. This approach is limited to problems of clamped boundaries and high polynomial degrees and suffers significantly from an ill-conditioned system of equations which influence both the solution process and the desired accuracy level. A classical penalty approach is used in [39, 48] for linear elasticity and in [49] for large-deformation analysis with a limitation to boundary fitted embedding domains. In RUESS ET AL. [44], weak boundary conditions are applied for bone mechanics analysis based on the p-version FCM. SCHILLINGER ET AL. [46, 49] and ZANDER ET AL. [57] apply the concept to enforce homogeneous boundary conditions in the p-version and B-spline version of the FCM, respectively. Both address the topic as part of a simulation concept that is based on an empirical choice for the required stabilization terms. In this contribution we extend the aforementioned theoretical formulation by several aspects to provide a complete picture on the weak boundary condition formulation. In particular we split the formulation according to the material properties of the solution domain to account for a proper choice of the method's stability terms that allow optimal convergence rates as stated in [10] for fluid dynamics problems. Furthermore, the formulation considers separate terms for the normal and shear part of the flux along the boundary to adjust the extension terms according to their mechanical properties. The type of essential boundary condition and the degree of the introduced restriction remain free parameters of the proposed formulation as demonstrated with several examples. We present an extensive range of benchmark problems, including scalar Laplace problems and vector problems from elasticity, that demonstrate numerical properties, accuracy aspects, simplicity and validity of the presented methods. We further illustrate the importance of an adequate choice of the stabilization terms carrying out extensive sensitivity studies. Different integration methods for cut cells are tested and evaluated with regard to their effects on the accuracy of the method in conjunction with weakly enforced boundary conditions. Furthermore, we show the impact of the relation between physical and fictitious domain parts in cut cells on the conditioning of the resulting system matrix. We demonstrate the potential of the method to obtain optimal rates of convergence under hrefinement and at least pre-asymptotically exponential rates of convergence under p-refinement. A comparison to reference solutions of classical finite element models for thin plates discloses the superiority of the proposed concept with regard to accuracy and flexibility in the modelling process. We conclude our study with two complex three-dimensional examples, a STL-based crankshaft model and a CT scan-based human femur, to motivate possible application areas for the presented methods and to underline their corresponding advantages. Finally, we demonstrate the value of the presented concepts with regard to trimmed NURBS geometries within isogeometric analysis.

Beyond the issue of weak boundary conditions, we present a novel integration strategy in the cut cells that reduces the integration cost of the finite cell method by one half. Furthermore we illustrate a composed integration scheme based on a locally confined quadrisection of triangles 
to specify boundary conditions arbitrarily, independent of the finite cell discretization.

In the following contribution we favour the B-spline and NURBS-based variant of the finite cell method to exploit favorably their smoothness and higher order continuity properties that in many cases lead to an increased per-degree-of-freedom accuracy. This observation is consistent with numerous results for isogeometric structural $[18,17]$, fluid [1], and fluid-structure interaction $[8,9]$ analyses, which also employ higher-order continuous basis functions. However, we would like to stress that the presented formulations and advantages of the weak imposition of essential boundary conditions equivalently also hold for other FCM versions and isogeometric-analysisbased concepts.

The paper is structured as follows. We provide a basic formulation of the FCM using the BSpline/NURBS discretization in Section 2. We present the weak boundary condition formulation for the FCM in Section 3. We provide numerical examples that support the good performance of the proposed methodology in Section 4 and summarize the main findings and draw conclusions in Section 5. 


\section{Basic formulation}

A summary of the principles of the finite cell method for problems of linear elasticity is provided in this section. The theory and methodology of this fictitious domain approach is formulated for solids on the basis of the principle of virtual work, independent of the applied Ansatz space. A detailed description of the method, particularly with specialization to Legendre-based Ansatz spaces [4, 51] or NURBS [41], can be found for example in [49, 44] and [42], respectively. In the following we apply B-splines and NURBS as a suitable approximation basis for a BubnovGalerkin formulation of the linear elasticity problem.

\subsection{The finite cell approach}

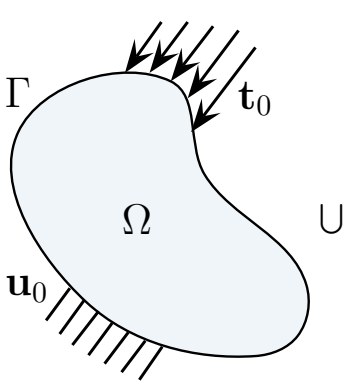

(1)

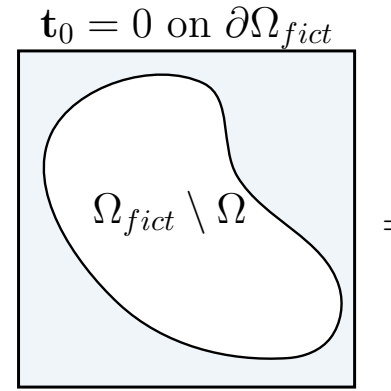

$(2)$

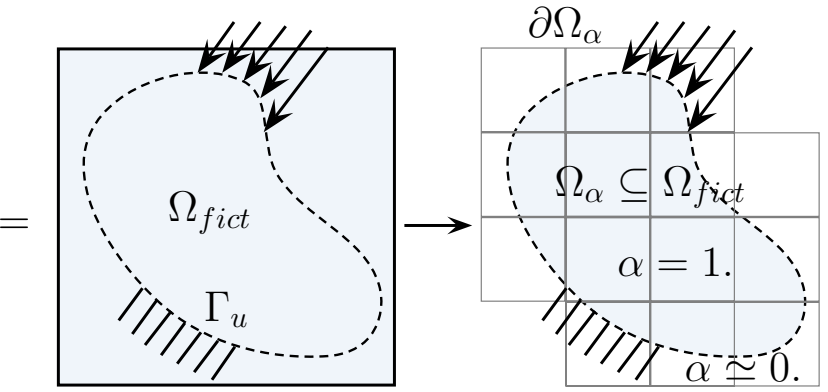

(3)

$(4)$

Figure 1: (1) Physical domain $\Omega$ with prescribed traction $\mathbf{t}_{0}$ along the Neumann boundary $\Gamma_{t}$ and prescribed displacements $\mathbf{u}_{0}$ along the Dirichlet boundary $\Gamma_{u}$, (2) fictitious domain $\Omega_{\text {fict }} \backslash \Omega$ with zero traction $\mathbf{t}_{0}$ on the cell domain surface $\partial \Omega_{f i c t}$, (3) embedded domain with implicit domain support for $\Omega_{\text {fict }}$ from prescribed displacement constraints on $\Gamma_{u}$ and (4) finally applied cell grid structure on $\Omega_{\alpha}=\Omega \cup \Omega_{e x t} \subseteq \Omega_{\text {fict }}$ with location function $\alpha(\mathbf{x})$.

Embedding the physical domain of interest $\Omega$ in an extended domain $\Omega_{\text {fict }}$ of much simpler shape, the finite cell method satisfies the weak formulation of the elasticity problem according to the principle of virtual work. The governing integral equations of the formulation are evaluated on $\Omega$ only, using a refined numerical integration scheme that captures the true boundary $\Gamma$ (Fig. 1). To reduce modelling effort the simulation domain $\Omega_{\alpha} \subseteq \Omega_{\text {fict }}$ is often chosen on a Cartesian grid thus taking advantage of the simple rectangular shape and a linear mapping to the normalized standard element. The fictitious domain approach is not necessarily restricted to the Cartesian grid and is also applicable for more general extension domains including mapped geometries [44, 39].

The boundary of the extended cell domain $\partial \Omega_{\alpha}$ is assumed traction free. Traction forces $\mathbf{t}$ are directly applied to the boundary of the true physical domain $\Gamma_{t}$ by

$$
\mathbf{t}(\mathbf{x})=\mathbf{t}_{0} \quad \forall \mathbf{x} \in \Gamma_{t}
$$

Preprint, submitted to Int. J. Numer. Meth. Engng. in 2012 
with prescribed values $\mathbf{t}_{0}$. In analogy with the Neumann boundary $\Gamma_{t}$, prescribed displacements $\mathbf{u}_{0}$ are defined along the Dirichlet boundary $\Gamma_{u}$

$$
\mathbf{u}(\mathbf{x})=\mathbf{u}_{0} \quad \forall \mathbf{x} \in \Gamma_{u}
$$

with $\mathbf{u}$ denoting the displacement vector on $\Omega_{\alpha}$, thus completing the boundary description of the solution domain $\Omega$ :

$$
\Gamma=\Gamma_{u} \cup \Gamma_{t} \quad \wedge \quad \Gamma_{u} \cap \Gamma_{t}=\emptyset
$$

The stress distribution within the embedding cell domain $\Omega_{\alpha}$ is chosen to be dependent on a location/penalization factor $\alpha$. For the relation between stresses and strains follows for linear elasticity

$$
\sigma_{\alpha}(\mathbf{x})=\mathbf{C}_{\alpha}(\mathbf{x}): \boldsymbol{\epsilon}(\mathbf{x})
$$

with $\boldsymbol{\epsilon}(\mathbf{x})$ denoting the linear strain tensor and $\mathbf{C}_{\alpha}(\mathbf{x})$ the elasticity tensor defined as

$$
\mathbf{C}_{\alpha}(\mathbf{x}):=\alpha \mathbf{C}, \quad\left\{\begin{array}{l}
\alpha=1\left(:=\alpha_{\Omega}\right) \quad \forall \mathbf{x} \in \Omega \\
\alpha=\gamma\left(:=\alpha_{e x t}\right) \quad \forall \mathbf{x} \in \Omega_{\text {ext }}
\end{array}\right.
$$

For points inside $\Omega$ the elasticity tensor $\mathbf{C}_{\alpha}$ represents the domain's material properties. For points in $\Omega_{\text {ext }}$, that are not contained in $\Omega$, the factor $\alpha$ penalizes $\mathbf{C}$ by a very small value $\gamma$ to confine the influence of the extension domain. The choice of $\alpha$ is a trade-off between accuracy and stability widely to prevent an ill-conditioned system of equations and to ensure convergence to the exact solution. Typical values for $\gamma$ range in the interval $\left[10^{-4}, 10^{-14}\right]$ and can be chosen reliably according to the problem's elasticity parameters e.g. $\gamma=(\lambda+\mu) \cdot \varepsilon^{*}$ with $\lambda$ and $\mu$ being the Lamé parameter [6].

In analogy to the stress distribution the volume forces of $\Omega_{e x t}$ are penalized

$$
\mathbf{p}_{\alpha}(\mathbf{x})=\alpha \mathbf{p}(\mathbf{x})
$$

Using (3), (4) and (5) follows the weak formulation for the physical domain $\Omega$ within $\Omega_{\alpha}$ according to the principle of virtual work

$$
\mathcal{W}(u, \delta u)=\mathcal{W}_{I}(u, \delta u)+\mathcal{W}_{E}(u, \delta u)=0
$$

with integral terms for the internal and external work, respectively

$$
\begin{aligned}
& \mathcal{W}_{I}=\int_{\Omega_{\alpha}} \delta \boldsymbol{\epsilon}: \boldsymbol{\sigma}_{\alpha} d v \\
& \mathcal{W}_{E}= \int_{\Omega_{\alpha}} \delta \mathbf{u}^{T} \mathbf{p}_{\alpha} d v+\int_{\Gamma_{t}} \delta \mathbf{u}^{T} \mathbf{t}_{0} d a \\
& \mathbf{x} \in \Gamma_{u} \Rightarrow \mathbf{u}=\mathbf{u}_{0}
\end{aligned}
$$

where $\delta \boldsymbol{\epsilon}$ denotes the variation of the strain tensor with respect to the virtual displacements $\delta \mathbf{u}$. With $\Gamma=\left\{\Gamma_{u} \cup \Gamma_{t}\right\} \subset \Omega_{\alpha}$ equations (7) and (8) substituted into (6) are a consistent weak formulation for the linear elasticity problem of $\Omega$ on $\Omega_{\alpha}$.

${ }^{*} \varepsilon$ : IEEE 754 standard unit roundoff/macheps $=2^{-53} \approx 1.11 \cdot 10^{-16}$ 


\subsection{B-spline discretization}

The finite cells are implemented as hexahedral elements according to the usual principles of finite elements using a tensor product space $[16,6]$.

In $1 \mathrm{D}$, a B-spline basis of polynomial degree $p$ is specified by $n$ basis functions $N_{i, p}(\xi)(i=$ $1, \ldots, n)$, each generated from $p+2$ knots $\xi_{i} \leq \xi_{i+1} \leq \ldots \leq \xi_{i+p+1}$ in the parameter space $\xi$. The knots are a non-decreasing set of points subdividing the parameter space $\xi$ into knot spans. The set $\Xi=\left\{\xi_{1}, \xi_{2}, \ldots, \xi_{n+p+1}\right\}$ is known as the knot vector. Repeated knots lower the continuity of the basis functions $N_{i, p}(\xi)$ between knot spans. A multiplicity of $p+1$ for knot $\xi_{i}$ ensures the corresponding basis function $N_{i, p}(\xi)$ is interpolatory at $\xi_{i}$. The basis functions of a patch are interpolatory at the first and last knot $\xi_{1}$ and $\xi_{n+p+1}$, respectively. The corresponding knot vector is said to be open (cf Fig. 2). The knot spans of a B-spline patch are analogous to a subdomain of finite elements and will be referred to in the following as knot-span-elements. The basis functions $N_{i, p}(\xi)$ are $\mathcal{C}^{p-1}$ continuous across the knot-span-elements and can be generated recursively by the Cox-de-Boor formula $[41,16]$.

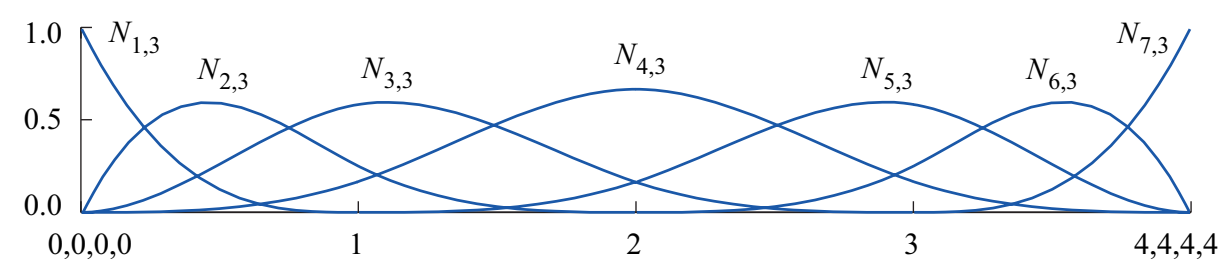

Figure 2: 1D cubic B-spline shape functions $N_{i, 3}(i=1, \ldots, 7)$ across an open knot vector of four knot-span-elements.

The multivariate B-spline basis of the finite cells is constructed by the Cartesian product $\Xi \times$ $\mathcal{H} \times \mathcal{Z}$ of $1 \mathrm{D}$ basis functions, defined by the knot vectors $\Xi=\left\{\xi_{1}, \xi_{2}, \ldots, \xi_{n+p+1}\right\}, \mathcal{H}=$ $\left\{\eta_{1}, \eta_{2}, \ldots, \eta_{m+p+1}\right\}$ and $\mathcal{Z}=\left\{\zeta_{1}, \zeta_{2}, \ldots, \zeta_{l+p+1}\right\}[16,30]$. Each shape function is specified as

$$
Q_{i j k, p}(\xi, \eta, \zeta)=N_{i, p_{1}}(\xi) M_{j, p_{2}}(\eta) L_{k, p_{3}}(\zeta)
$$

where $N_{i, p_{1}}(\xi), M_{j, p_{2}}(\eta)$ and $L_{k, p_{3}}(\zeta)$ are the basis functions of polynomial degree $p_{s}$ in each parametric direction $s \in\{1,2,3\}$, respectively, and where $i, j$ and $k$ indicate the position of the basis function within the product space.

The shape functions (9) are used to specify the Ansatz for the interpolation of the displacement field and corresponding derivatives

$$
\mathbf{u}=\mathbf{Q}^{T}(\xi, \eta, \zeta) \mathbf{U}
$$

with $\mathbf{U}^{T}=\left[U_{1} \ldots U_{N}\right]$ representing the introduced degrees of freedom in terms of global displacements of the control points controlling the structure's geometry and $\mathbf{Q}(\xi, \eta, \zeta)\left(:=\left[Q_{i j k, p}(\xi, \eta, \zeta)\right]\right)$ the interpolation matrix assembled from the shape functions of (9). 
The strain tensor $\epsilon$ in (7) is approximated as

$$
\hat{\boldsymbol{\epsilon}}^{\dagger}=\mathbf{B}^{T}(\xi, \eta, \zeta) \mathbf{U},
$$

where $\mathbf{B}(\xi, \eta, \zeta)$ is the strain-displacement matrix [6].

\subsection{Improved numerical integration concept on a cell level}

Gaussian integration on sub-cells is applied for the integrals in (7) and (8). This composed integration scheme [55] allows us to densify the quadrature points arbitrarily according to the structural needs of the geometric or physical configuration by an independent cell decomposition into smaller units of arbitrary size thus confining the integration error of the implicit domain representation. The approach also proved for producing excellent results for heterogeneous material distributions [55, 44].

The sub-cell scheme can be restricted to boundary cells for homogeneous material properties to capture the true boundary of the physical domain $\Omega$, whereas cells that are completely inside the domain are treated as standard hexahedral. A tree-based decomposition strategy of the cell domain is applied favorably (Fig. 3) to reduce the integration effort.

The sub-cell approach is restricted to the cell level and does not require expensive global data

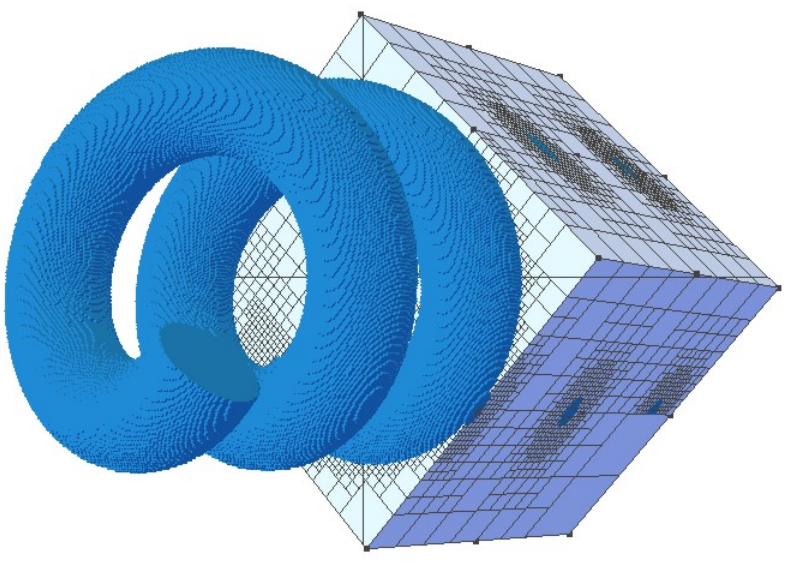

Figure 3: Octree-based cell decomposition into sub-cells for boundary cells of homogeneous material.

structures. A large number of sub-cells applied to a small number of quadrature points is also the preferred strategy for higher polynomial degrees essentially to economize the numerical cost of the numerical integration.

With the strain-displacement matrix $\mathbf{B}(\xi, \eta, \zeta)$ and the location-dependent material stiffness tensor $\mathrm{C}_{\alpha}$ the cell stiffness matrix is computed by the integral

$$
\mathbf{K}_{c}=\sum_{s c}\left\{\int_{\xi} \int_{\eta} \int_{\zeta} \mathbf{B}^{T} \mathbf{C}_{\alpha} \mathbf{B} \operatorname{det}\left(\mathbf{J}_{c}\right) \operatorname{det}\left(\mathbf{J}_{s c}\right) d z_{1} d z_{2} d z_{3}\right\}
$$

\footnotetext{
${ }^{\dagger} \hat{\epsilon}$ : Voigt notation for the strain $\epsilon$
} 
with $\mathbf{J}_{c}^{\ddagger}$ and $\mathbf{J}_{s c}{ }^{\S}$ representing the Jacobian of the finite cell and its sub-cells, respectively. The strain-displacement matrix $\mathbf{B}$ is evaluated for each sub-cell with respect to the mapping of the sub-cell coordinates of a locally defined Cartesian coordinate system $\left(z_{1}, z_{2}, z_{3}\right)$ located in the sub-cell center to the local coordinate system of the finite cell.

$$
\mathbf{B}=\mathbf{B}\left(\xi\left(z_{1}\right), \eta\left(z_{2}\right), \zeta\left(z_{3}\right)\right)
$$

The material stiffness tensor $\mathbf{C}_{\alpha}$ is evaluated at each integration point within the subcells. The evaluation of the load integral of (8) follows in analogy with the integration of the stiffness matrix.
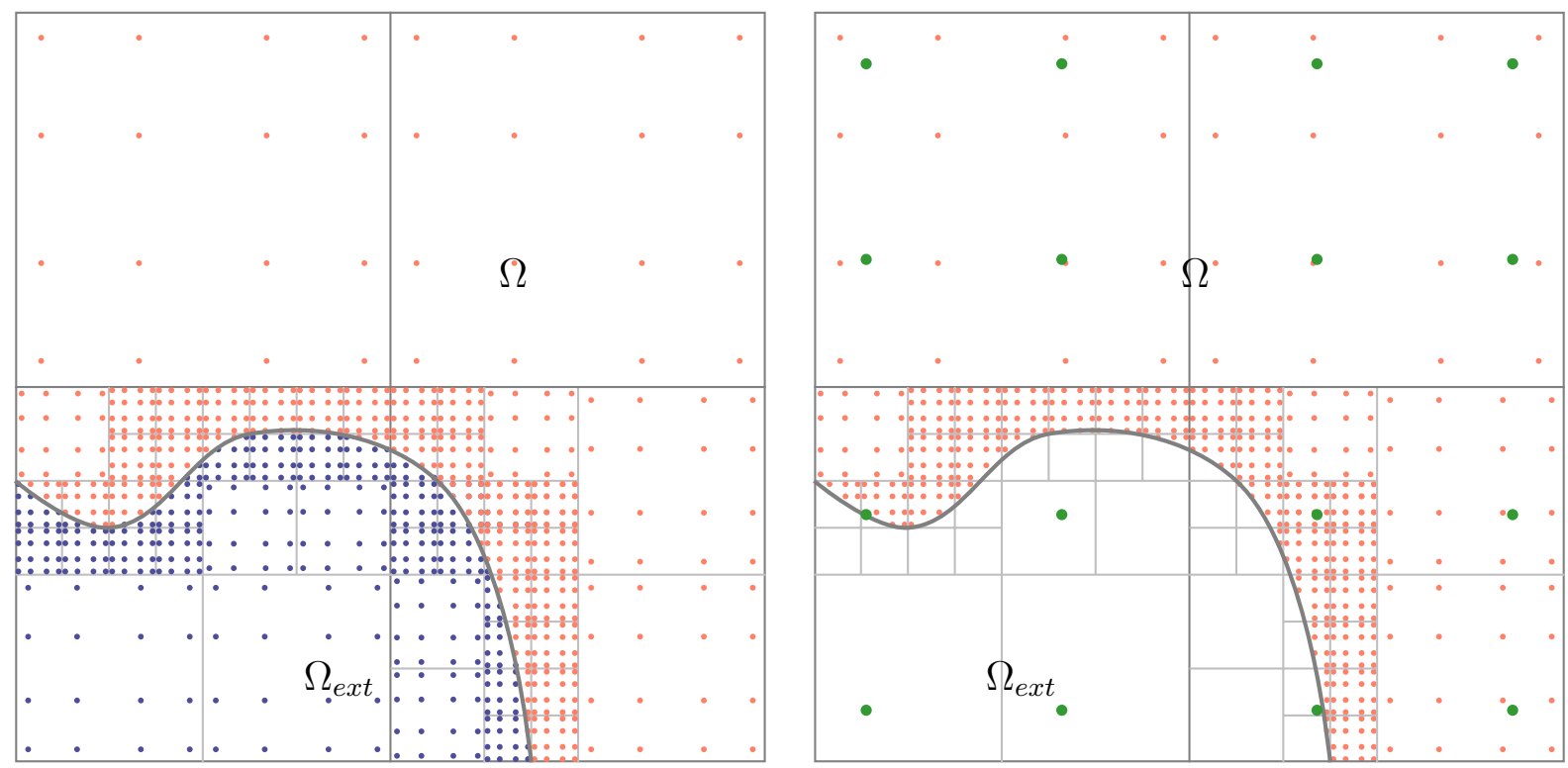

Figure 4: Reduced integration concept for cut boundary cells.

An efficient strategy for the composed integration concept that significantly reduces the numerical effort is illustrated in Figure 4. To the left a quadtree-based sub-cell decomposition of a cut boundary cell is shown. Each sub-cell is integrated with $p+1$ quadrature points. The applied recursive bisection approach densifies the number of sub-cells and thus the number of integration points in the vicinity of the domain boundary $\Gamma$. The stresses inside $\Omega$ are multiplied at each integration point (red points) with $\alpha_{\Omega}=1.0$ to account for their full contribution to the governing elasticity equations, resulting in a stiffness contribution $\mathbf{K}_{\Omega}$. Stresses at the integration points of the extension domain (blue points) are multiplied with, e.g., $\alpha_{\text {ext }}=10^{-14}$, resulting in a stiffness contribution $\mathbf{K}_{\Omega_{\alpha}}$, thus fading out any significant contribution from the extension domain $\Omega_{\text {ext }}$.

$$
\mathbf{K}_{c}=\mathbf{K}_{\Omega}\left(\alpha_{\Omega}\right)+\mathbf{K}_{\Omega_{e x t}}\left(\alpha_{e x t}\right)
$$

\footnotetext{
${ }_{\ddagger}^{\ddagger}$ cell index $\{\cdot\}_{c}$

${ }^{\S}$ sub-cell index $\{\cdot\}_{s c}$
}

Preprint, submitted to Int. J. Numer. Meth. Engng. in 2012 
Instead of a numerically demanding sub-cell integration in both domains, $\Omega$ and $\Omega_{e x t}$, a modified integration concept is followed that reuses the integration result of the true domain to determine the stiffness contribution of the extension domain as illustrated to the right of Figure 4.

$$
\mathbf{K}_{c}=\mathbf{K}_{c}\left(\alpha_{\text {ext }}\right)+\mathbf{K}_{\Omega}\left(\alpha_{\Omega}-\alpha_{\text {ext }}\right)
$$

In a first step a stiffness matrix contribution for the complete cell domain is computed applying the factor $\alpha_{\text {ext }}$. In the second step, a composed sub-cell integration that is restricted to the true domain $\Omega$ of the cell is performed with $\left(\alpha_{\Omega}-\alpha_{e x t}\right)$ and added to the cell stiffness matrix.

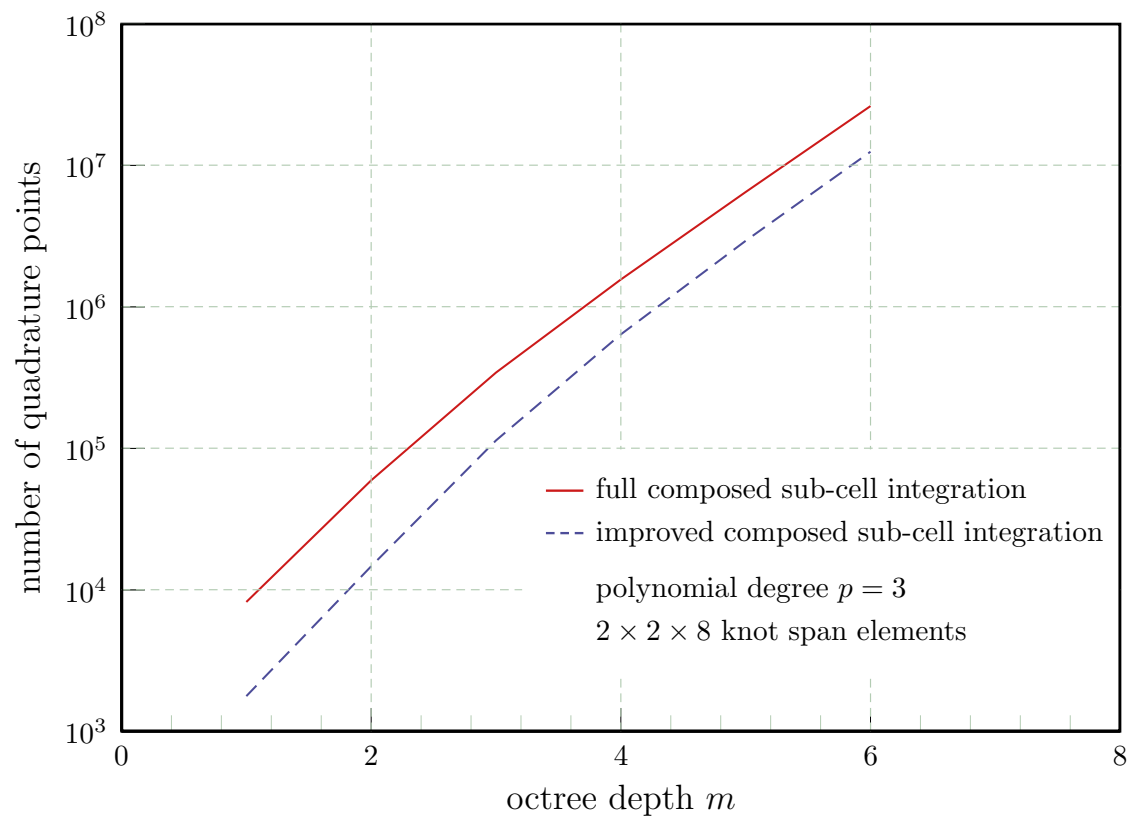

Figure 5: Classical [19] vs. modified integration concept for the embedded spring model of Fig 3.

The reduction of the integration effort for the embedded spring model (Fig. 3) is illustrated in Figure 5 in terms of quadrature points that are placed at various octree depths to evaluate the stiffness matrix with both methods, the classical composed sub-cell integration scheme [49] and the improved scheme that profits from the identification process of the interior of $\Omega$. With an increasing octree depth, for this specific problem, the decreasing factor for the improved integration concept reduces from $4.5(p=1)$ to a value of $2.1(p=6)$, still doubling the efficiency of the stiffness matrix computation.

\section{Weakly enforced essential boundary conditions}

The elastic equilibrium (6) is consistently extended to a formulation that enforces the essential boundary conditions in a weak sense. The expression in (8) that satisfies the Dirichlet boundary

Preprint, submitted to Int. J. Numer. Meth. Engng. in 2012 
conditions pointwise,

$$
\mathbf{u}_{0}-\mathbf{u}=\mathbf{0} \quad \text { for } \mathbf{x} \in \Gamma_{u},
$$

is replaced by the weighted residual term

$$
\int_{\Gamma_{u}} \delta \mathbf{t}^{T}\left(\mathbf{u}_{0}-\mathbf{u}\right) d a=0
$$

The weight function $\delta \mathbf{t}=\delta(\boldsymbol{\sigma} \mathbf{n})$ is chosen to ensure consistency in the physical dimension of the equilibrium (6), where $\mathbf{n}$ is the outward unit normal along the boundary $\Gamma$. The formulation retains symmetry by adding the weighted residual of the unknown reaction forces $\mathbf{t} \in \Gamma_{u}$ along the Dirichlet boundary in Equation (7)

$$
-\int_{\Gamma_{u}} \delta \mathbf{u}^{T}(\mathbf{t}) d a=-\int_{\Gamma_{u}} \delta \mathbf{u}^{T}(\boldsymbol{\sigma} \mathbf{n}) d a .
$$

Introducing equations (16) and (17) requires stabilization of the formulation to ensure coercivity of (6) thus retaining positive definiteness of the resulting system matrix by the introduction of a stability term

$$
\int_{\Gamma_{u}} \tau \delta \mathbf{u}^{T}\left(\mathbf{u}_{0}-\mathbf{u}\right) d a
$$

with a stability factor $\tau$. In $[20,28,25]$ it is shown that for $\tau$ chosen large enough the discrete solution converges to the exact solution in optimal order with respect to the $H^{1}$ - and $L^{2}$-norm. A proper choice of $\tau$ can be found in dependence of the characteristic length $h$ of the discretization and a large enough constant $C$. With a sufficiently large value for $\tau$, the extended weak form results in a positive definite problem that ensures convergence if the inequality

$$
\left\|h^{1 / 2} \boldsymbol{\sigma}(\delta \mathbf{u}) \mathbf{n}\right\|_{L_{2}(\Gamma)}^{2} \leq C\|\boldsymbol{\sigma}(\delta \mathbf{u})\|_{L_{2}\left(\Omega_{C}\right)}^{2}
$$

is satisfied for all $(\delta \mathbf{u})$ of the interpolation space and $\tau>C[28,10,25,22]$. A suitable choice of the stability parameter is further discussed in section 4 .

\subsection{Extension of the Principle of Virtual Work}

HANSBO ET AL.[28] and BAZILEVS ET AL.[10] show that for optimal convergence the stability term has to be chosen directly proportional to the material parameters of the problem, and inversely proportional to the mesh size $h$ in direction of the boundary normal. Following this, we split $\tau$ into a part normal ${ }^{\mathbb{}}$ and tangential ${ }^{\|}$to the domain boundary. In a second step we introduce material dependent stability parameters considering the material's bulk and shear modulus.

\footnotetext{
9index $N$

"index $S$
}

Preprint, submitted to Int. J. Numer. Meth. Engng. in 2012 
With definitions (16), (17) and (18) the extension of the principle of virtual work has the following form:

$$
\begin{array}{r}
\mathcal{W}_{I}=\int_{\Omega_{\alpha}} \delta \boldsymbol{\epsilon}: \boldsymbol{\sigma}_{\alpha} d v-\int_{\Gamma_{u}} \delta(\boldsymbol{\sigma} \mathbf{n})^{T} \mathbf{u} d a-\int_{\Gamma_{u}} \delta \mathbf{u}^{T}(\boldsymbol{\sigma} \mathbf{n}) d a \\
+\int_{\Gamma_{u}} \tau_{S} \delta \mathbf{u}^{T} \mathbf{u} d a+\int_{\Gamma_{u}} \tau_{N}\left(\mathbf{n}^{T} \delta \mathbf{u}\right)\left(\mathbf{u}^{T} \mathbf{n}\right) d a \\
\mathcal{W}_{E}=\int_{\Omega_{\alpha}} \delta \mathbf{u}^{T} \mathbf{p}_{\alpha} d v+\int_{\Gamma_{t}} \delta \mathbf{u}^{T} \mathbf{t}_{0} d a-\int_{\Gamma_{u}} \delta(\boldsymbol{\sigma} \mathbf{n})^{T} \mathbf{u}_{0} d a \\
+\int_{\Gamma_{u}} \tau_{S} \delta \mathbf{u}^{T} \mathbf{u}_{0} d a+\int_{\Gamma_{u}} \tau_{N}\left(\mathbf{n}^{T} \delta \mathbf{u}\right)\left(\mathbf{u}_{0}^{T} \mathbf{n}\right) d a
\end{array}
$$

with $(\delta \boldsymbol{\epsilon}: \boldsymbol{\sigma}(\alpha))=\sum_{i} \sum_{j} \delta \epsilon_{i j} \sigma_{i j}$ and $\tau_{N}$ and $\tau_{S}$ denoting the penalty parameter with respect to the shear and normal part of the boundary integrals, respectively.

Equations (20) and (21) for isotropic material properties split into terms dependent on the Lamé parameter $\lambda$ and $\mu$ :

$$
\begin{aligned}
\lambda & :=E \cdot \nu /((1+\nu)(1-2 \nu)) \\
\mu & :=E /(1+\nu)
\end{aligned}
$$

with Young's modulus E and Poisson ratio $\nu$. With (22) and (23) the stress tensor follows as

$$
\begin{aligned}
& \boldsymbol{\sigma}=\lambda \nabla \cdot \mathbf{u} \mathbf{I}+2 \mu \boldsymbol{\epsilon} \quad \text { with } \\
& \sigma_{i j}=\lambda u_{k, k} \delta_{i j}+\mu\left(u_{i, j}+u_{j, i}\right)
\end{aligned}
$$

where $\nabla \cdot \mathbf{u}=\operatorname{tr}(\boldsymbol{\epsilon})$.

Substitution of (24) into (20) and (21) and separation of the terms with regard to the Lamé parameter results in a formulation that allows a proper choice of the penalty values $\tau_{S}$ and $\tau_{N}$ subject to $\mu$ and $\lambda$.

$$
\begin{gathered}
\mathcal{W}_{I}=\int_{\Omega_{\alpha}} \delta \boldsymbol{\epsilon}: \boldsymbol{\sigma}_{\alpha} d v-\lambda \int_{\Gamma_{u}} \delta(\nabla \cdot \mathbf{u I n})^{T} \mathbf{u} d a-\lambda \int_{\Gamma_{u}} \delta \mathbf{u}^{T}(\nabla \cdot \mathbf{u I n}) d a \\
+\int_{\Gamma_{u}} \tau_{N}\left(\mathbf{n}^{T} \delta \mathbf{u}\right)\left(\mathbf{u}^{T} \mathbf{n}\right) d a-\mu \int_{\Gamma_{u}} \delta(\boldsymbol{\epsilon} \mathbf{n})^{T} \mathbf{u} d a-\mu \int_{\Gamma_{u}} \delta \mathbf{u}^{T}(\boldsymbol{\epsilon} \mathbf{n}) d a \\
+\int_{\Gamma_{u}} \tau_{S} \delta \mathbf{u}^{T} \mathbf{u} d a \\
\mathcal{W}_{E}=\int_{\Omega_{\alpha}} \delta \mathbf{u}^{T} \mathbf{p}_{\alpha} d v+\int_{\Gamma_{t}} \delta \mathbf{u}^{T} \mathbf{t}_{0} d a-\lambda \int_{\Gamma_{u}} \delta(\nabla \cdot \mathbf{u I} \mathbf{n})^{T} \mathbf{u}_{0} d a \\
+\int_{\Gamma_{u}} \tau_{N}\left(\mathbf{n}^{T} \delta \mathbf{u}\right)\left(\mathbf{u}_{0}^{T} \mathbf{n}\right) d a+\int_{\Gamma_{u}} \tau_{S} \delta \mathbf{u}^{T} \mathbf{u}_{0} d a-\mu \int_{\Gamma_{u}} \delta(\boldsymbol{\epsilon} \mathbf{n})^{T} \mathbf{u}_{0} d a
\end{gathered}
$$

Preprint, submitted to Int. J. Numer. Meth. Engng. in 2012 
The last four additional terms in (27) have no contribution to the weak formulation for homogeneous boundary conditions. The equilibrium (6) is ensured by additional terms in (26) to balance the external forces with the reaction forces along the constraint boundary. Furthermore, neglecting the Poisson effect $(\nu=0)$ remains only those additional terms in the formulation that are associated with Lamé's second parameter, the shear modulus $\mu$. With $\nu \neq 0$ the need for separation according to the normal and tangential constituents of the stress tensor and the introduction of separate stability terms controlled by $\tau_{N}$ and $\tau_{S}$ becomes evident with regard to optimal stabilization. For nearly incompressible materials $\left(\nu \approx \frac{1}{2}\right) \tau_{N} \gg \tau_{S}$. As a result the normal component of the displacements gets a heavier penalty.

\subsection{Stabilization aspects}

According to $[10,28]$ we choose the stability parameters $\tau_{N}$ and $\tau_{S}$ with regard to $\lambda$ and $\mu$, respectively, the local mesh size $h$ and constants $C_{N}$ and $C_{S}$, which only depend on the polynomial order $p$ of the interpolation space.

$$
\begin{aligned}
\tau_{N} & =C_{N}(p) \frac{\lambda}{h}, \\
\tau_{S} & =C_{S}(p) \frac{\mu}{h} .
\end{aligned}
$$

The stability terms (28) and (29) are influenced by the regularity of the element mesh. Mesh regularity is ensured for the finite cell method by the Cartesian grid of the embedding domain. Unfortunately this benefit is relativized in many cases by the presence of finite cells that are cut by the boundary. Such cells often cover the solution domain only with a small percentage of their cell domain. The small but significant stiffness contribution of such cells strongly affects the conditioning of the overall system stiffness. This effect is amplified by the boundary contributions of the weak boundary formulation (20). Whereas the stresses of the fictitious domain are penalized with a value $\alpha$ close to the unit roundoff to mimic an embedding domain of nearly infinite softness, the stabilization of the boundary terms of (20) with $\tau_{N}$ and $\tau_{S}$ counteract this property and increases the condition number of the global stiffness. The following example illustrates this principle behavior of the conditioning of the elasticity problem in dependence of the dominating influence parameters such as cell size and share in the physical domain. The behavior for weakly and strongly enforced boundary conditions is compared.

A square domain $\Omega$ of unit size embedded in a finite cell discretization of varying extension domain is shown in Figure 6. Where $\Gamma_{u}$ denotes the embedded Dirichlet boundary with symmetry boundary conditions, $\Gamma_{t}$ denotes the Neumann boundary of prescribed traction. The domain $\Omega$ was placed at the upper left corner of the extension domain $\Omega_{e x t}$ for the computations with weakly satisfied boundary conditions, thus embedding the boundary $\Gamma_{u}$ within $\Omega_{\text {ext }}$ (Fig. 6). The reference model with strongly satisfied boundary conditions along $\Gamma_{u}$ was chosen to coincide with the boundary of $\Omega_{e x t}$ at the lower right corner.

The condition number $\mathcal{K}(\mathbf{K})\left(:=\|\mathbf{K}\| \cdot\left\|\mathbf{K}^{-1}\right\|\right)$ of the system stiffness is plotted in the diagram 6(b) for a variation of the extension domain between $a$ and $2 a$, for this example the factor $\alpha$ (Eq. (4)), which penalizes the stresses of the fictitious extension domain was chosen to be $10^{-12}$. 


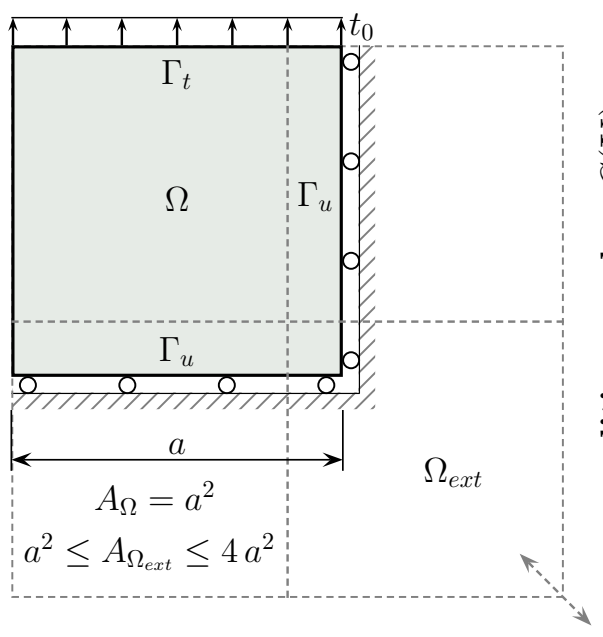

(a) problem definition

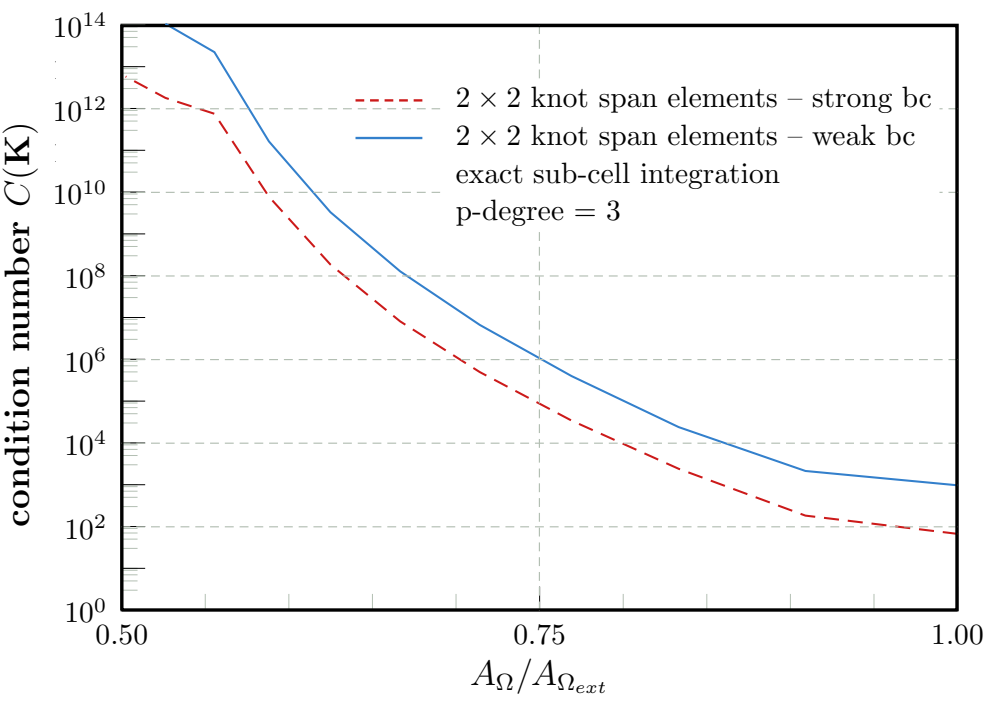

(b) condition number $\mathcal{K}(\mathbf{K})$

Figure 6: Cut boundary elements: influence on the system condition.

As expected $\mathcal{K}(\mathbf{K})$ increases rapidly as the domain contribution of the cut element shrinks towards zero. The condition number for the weak boundary formulation steadily increases at a slightly higher level (blue curve) compared to a problem formulation that satisfies the boundary conditions in a strong sense (red curve), an observation that is confirmed by other problems of higher complexity. Nevertheless, the condition numbers for the presented weak boundary formulation is still two orders of magnitude less than that for a pure penalty approach.

We use a direct solver for the solution of the linear systems of equations arizing in the finite cell method. In all our finite cell simulations the penalization factor $\alpha$ varies between $10^{-10}$ and $10^{-14}$ without revealing any severe problems with regard to stability and accuracy of the solution process. The same is also true for the more sensitive solution of the standard eigenvalue problem based on iterative subspace solvers [42].

A continuous increase of the extension domain also has a direct effect on the stability parameters $\tau_{N}\left(C_{N}\right)$ and $\tau_{S}\left(C_{S}\right)$ which essentially contribute to the conditioning of the weak boundary formulation. Figure 7 shows the evolution of $C_{S}$ (red curve) derived from a generalized eigenvalue problem $[25,28,43]$ comprising condition (19) for a stable estimate of $C_{S}$. The values $C_{N}$ are marginally smaller growing at similar rates. A proof on the stability of the formulation and the appropriate choice of the stability parameters satisfying (19) is shown in detail in [20, 27, 22, 25]. Bearing in mind the influence of cut cells on the performance of the method, adjusting the cell grid to avoid cells with a very small contribution to the system domain seems to be an inevitable consequence. Fortunately larger problems do not, in general, show a high sensitivity with regard to the choice of the stability parameters as expected from the aforementioned problem. Nevertheless, a global choice of $\tau_{N}$ and $\tau_{S}$ as proposed in $[25,26,22,32]$ often fails to provide reasonable values that do not degenerate the formulation to a pure penalty method. Instead we follow a local eigenvalue approach that was proposed by EMBAR ET AL. [20] to estimate $C_{N}$ and $C_{S}$. This way we keep the influence of strongly degenerated boundary cells local thus improving the ac- 
M. Ruess et al. - Weak Boundary Conditions for the FCM

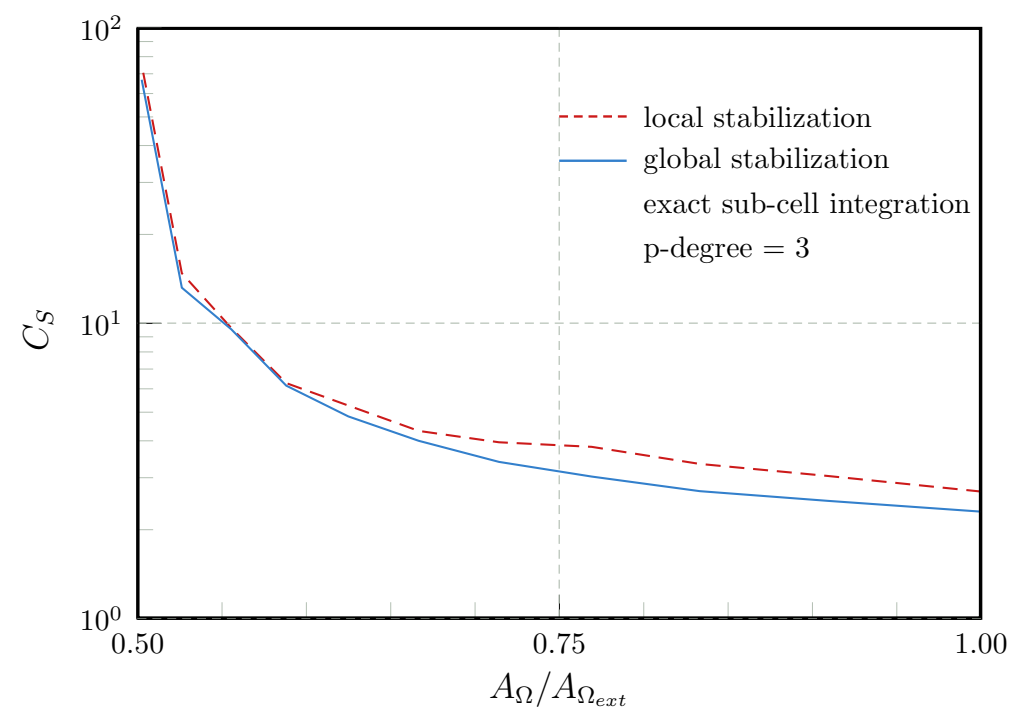

Figure 7: Cut boundary elements: development of $C_{S}$.

curacy in non-critical areas. Using relation (24) and the inequality (19), the eigenvalue problems to bound $C_{S}$ and $C_{N}$ are formulated as follows:

$$
\begin{aligned}
& \mathbf{A}_{*} \mathbf{x}=\hat{C}_{*} \mathbf{B}_{*} \mathbf{x} \quad *=[S, N] \\
& \text { with } \quad \mathbf{A}_{S}:=\int_{\Gamma}(\mathbf{B} \hat{\mathbf{n}})(\mathbf{B} \hat{\mathbf{n}})^{T} d \Gamma \quad \mathbf{B}_{S}:=\int_{\Omega} \mathbf{B} \mathbf{B}^{T} d \Omega \\
& \text { and } \quad \mathbf{A}_{N}:=\int_{\Gamma}(\nabla \mathbf{Q n})(\nabla \mathbf{Q n})^{T} d \Gamma \quad \mathbf{B}_{N}:=\int_{\Omega}(\nabla \mathbf{Q})^{T}(\nabla \mathbf{Q}) d \Omega
\end{aligned}
$$

respectively, where $\mathbf{x}$ represents any variation $\delta \mathbf{u}$ and $\hat{\mathbf{n}}$ is a matrix that represents the outward pointing normal components along $\Gamma, \mathbf{n}$ is the corresponding vector form of the normal and matrices $\mathbf{B}$ and $\mathrm{Q}$, as defined in section 2.2. The computationally intensive part of the eigenvalue problem refers to the domain integral of matrix $\mathbf{B}_{*}$ that requires the adaptive sub-cell integration (seesection 2.3) for boundary cut cells. Fortunately, the coefficients of $\mathbf{B}_{*}$ are known already from the computation of the cell-stiffness matrix $\mathbf{K}_{c}$ and are therefore reused. The computation of $\mathbf{A}_{*}$ is a ( $\left.\mathrm{d}^{* *}-1\right)$-dimensional problem requiring significantly less numerical effort than the finite cell computation itself. For boundary fitted cells the eigenvalue problem considers only boundary degrees of freedom. For boundary cut cells generally all degrees of freedom contribute to the problem. The solution of (30) includes only the eigenvalue of largest magnitude which is generally the dominant solution for Krylov methods such as the method of Lanczos or vector iteration [?, ?] and can be determined within a few iteration cycles.

The blue curve in Figure 7 provides the evolution of the proposed maximum local eigenvalue (31) for the critical lower right cell (cf Fig. 6). For boundary fitted problems it shows that

\footnotetext{
${ }^{* *} \mathrm{~d}$ : geometric dimension
} 
the local maximum eigenvalues are typically slightly bigger than their global counterparts, yet without noticeable significance in terms of accuracy. The local approach provides a simple and reliable mechanism for an automated choice of the stability parameters $\tau_{N}$ and $\tau_{S}$.

\subsection{Boundary integration}

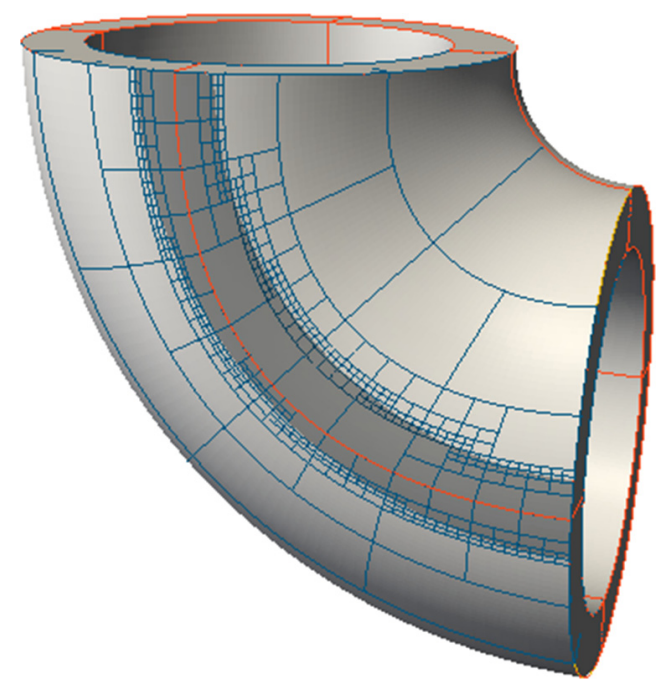

(a) sub-cell mesh

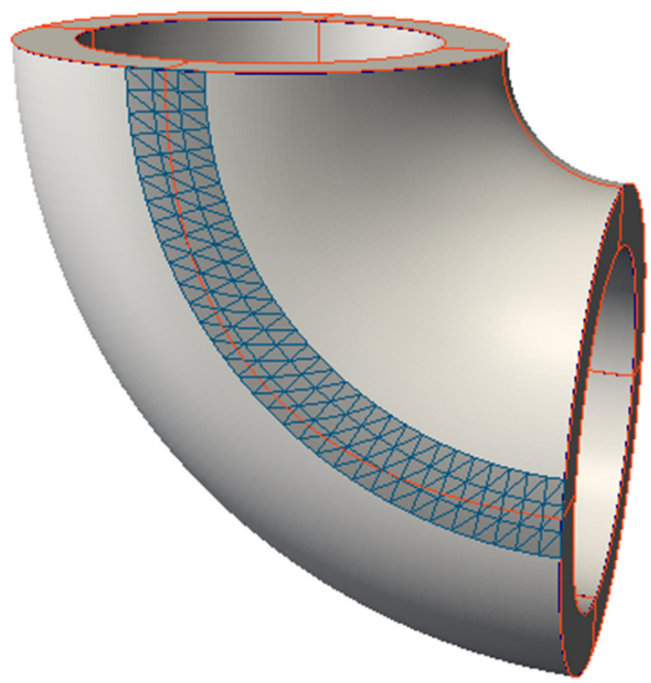

(b) triangle mesh

Figure 8: NURBS Dirichlet boundary strip of a 3D structure with variants: (a) quadtree-based sub-cell scheme and (b) triangulated boundary strip.

The additional integral terms of the extended formulation (26) and (27), entirely refer to the boundary of the physical domain $\Omega$ and require an adequate boundary description. In the following we will consider two different cases according to different strategies: In isogeometric analysis the NURBS representation of the analysis domain provides a geometrically exact boundary description which can be directly employed for the proposed method. In cases where only a part of the domain boundary is constrained, the fictitious domain approach in combination with adaptive integration as introduced in 2.1 is, applied to the structure's surface, a simple strategy to capture the desired boundary part accurately and to avoid a partial remodelling of the surface domain. Figure 8(a) illustrates the problem for a single NURBS patch. The surface again is a NURBS but only partially constrained. Instead of a separately defined overlay NURBS surface specifying the domain of enforced boundary conditions, the original surface is used in the sense of the finite cell approach. The sub-cells shown in Figure 8(a) are projected to the NURBS geometry for illustration purposes only. The quadtree is generated cell-wise in the parameter space $(\xi, \eta, \zeta)$, independent of adjacent cells.

It is often convenient to triangulate the surface patch or a desired subdomain thereof for piecewise linear boundary descriptions. Furthermore, a very fine mesh of triangles covering a curved 
surface domain is, in some cases, a sufficiently accurate approximation that can be quickly generated. Both cases, the NURBS-surface and the triangulated surface suffer from the need to match the outline boundary of the knot-span cells (cf Fig. 8(b)) to assign the surface contribution of the extension integrals (20) and (21) correctly to the corresponding knot-span cell.

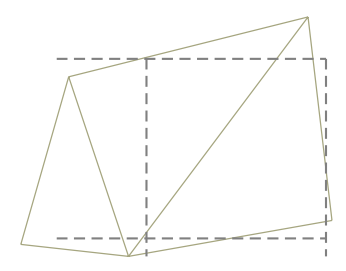

cut surface mesh

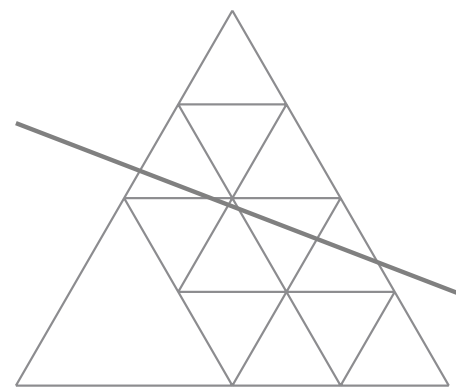

level 2

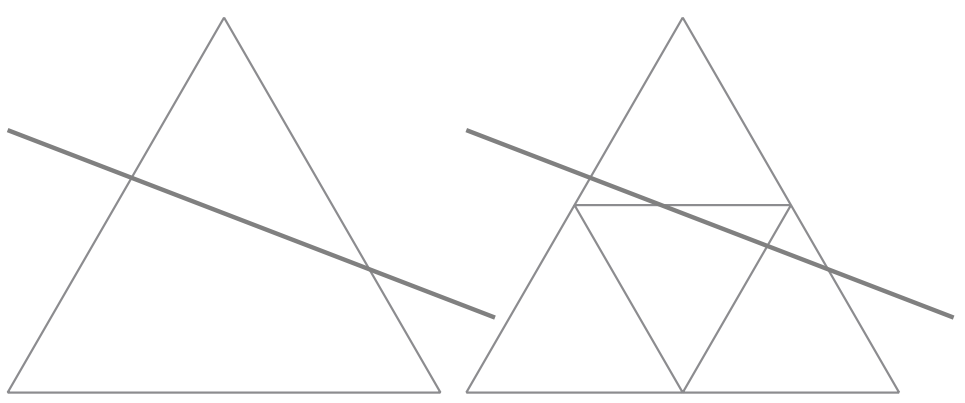

level 0

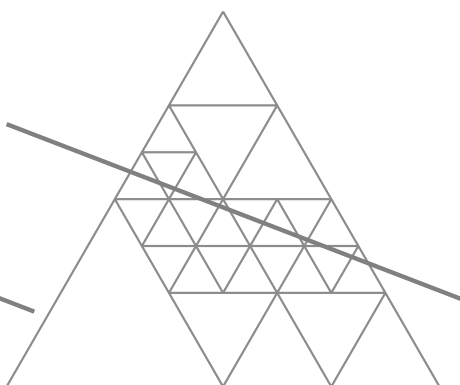

level 3 level 1

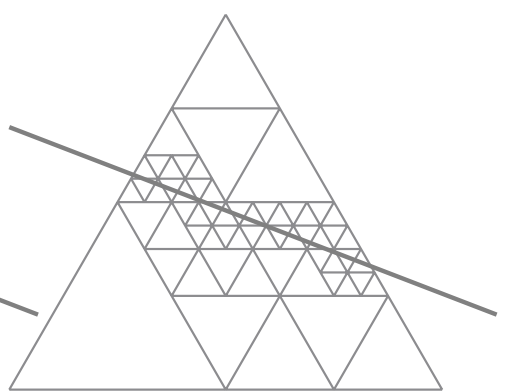

level 4

Figure 9: Recursive quadrisection of the surface mapped standard integration triangle.

Following the composed integration scheme set out in section 2.3 we approximate the boundary integrals using a recursive quadrisection of the standard triangle domain to account for a possible triangle overlap along the knot-span cell boundary. The quadrisection of each level into congruent triangular sub-cells is again a purely local approach, independent of adjacent cells and used for numerical integration only. Considering the relevant subset of surface triangles for each knot-span cell we apply Algorithm 1 to refine with sub-cells along the cutting lines through triangles. In analogy to the cell-wise sub-cell integration of 2.3 the recursion process results in a tree-structure that densifies quadrature points along the cutting line to gain control over the integration accuracy of the boundary domain.

The implementation of the recursive refinement algorithm is replaced by a non-recursive version using a fifo-queue to administrate the sub-cell divisions. 
M. Ruess et al. - Weak Boundary Conditions for the FCM

Data: subcell $\left(\hat{z}_{1}, \hat{z}_{2}, \hat{z}_{3}, h\right.$, level $):=$ normalized standard triangle with coordinates

$0 \leq z_{1}, z_{2}, z_{3} \leq 1$ and $z_{1}+z_{2}+z_{3}=1$, edge coordinates $\hat{z}_{1}=0, \hat{z}_{2}=0, \hat{z}_{3}=0$, triangle height $h=1$, current quadtree level level $=0$, max. quadtree depth max Level, fifo-queue $q$

Result: set of triangle sub-cells $s c$

if !subcell.isCut() then

$s c$.store(subcell) return

else

foursubcells $=\operatorname{subDivide}\left(\hat{z}_{1}, \hat{z}_{2}, \hat{z}_{3}, h / 2\right.$, level +1$)$

q.enqueue( four subcells)

while $q$ is not empty do

subcell $=$ q.dequeue;

if subcell.level $<$ maxLevel then

if !subcell.isCut() then

I sc.store(subcell)

else

foursubcells $=\operatorname{subDivide}\left(\right.$ subcell. $\hat{z}_{1}$, subcell. $\hat{z}_{2}$, subcell. $\hat{z}_{3}$, subcell.h/2,subcell.level +1 )

q.enqueue(four subcells)

end

else

I sc.store (subcell)

end

end

end

Algorithm 1: Sub-cell refinement algorithm for the standard integration triangle.

The generated sub-cells are stored and returned in a vector sc. A fifo-queue $q$ is the local datastructure to manage (enqueue/dequeue) the sub-cells of each subdivision step. The need for quadrisection is tested for each sub-cell of $q$. isCut() returns true, if quadrature points of the current subcell exist outside the knot-span cell domain. The method subDivide $\left(\hat{z}_{1}, \hat{z}_{2}, \hat{z}_{3}, h / 2\right.$, level+ 1) creates four sub-cells with edge coordinates $\hat{z}_{1}, \hat{z}_{2}, \hat{z}_{3}$ as follows:

$$
\begin{array}{ll}
\text { If } \hat{z}_{1}+\hat{z}_{2}+\hat{z}_{3}>1 & \text { Else } \\
\operatorname{subcell}\left(\hat{z}_{1}-\bar{h}, \hat{z}_{2}, \hat{z}_{3}, \bar{h}, \overline{l v}\right) & \operatorname{subcell}\left(\hat{z}_{1}+\bar{h}, \hat{z}_{2}, \hat{z}_{3}, \bar{h}, \overline{l v}\right) \\
\operatorname{subcell}\left(\hat{z}_{1}, \hat{z}_{2}-\bar{h}, \hat{z}_{3}, \bar{h}, \overline{l v}\right) & \operatorname{subcell}\left(\hat{z}_{1}, \hat{z}_{2}+\bar{h}, \hat{z}_{3}, \bar{h}, \overline{l v}\right) \\
\operatorname{subcell}\left(\hat{z}_{1}, \hat{z}_{2}, \hat{z}_{3}-\bar{h}, \bar{h}, \overline{l v}\right) & \operatorname{subcell}\left(\hat{z}_{1}, \hat{z}_{2}, \hat{z}_{3}+\bar{h}, \bar{h}, \overline{l v}\right) \\
\operatorname{subcell}\left(\hat{z}_{1}-\bar{h}, \hat{z}_{2}-\bar{h}, \hat{z}_{3}-\bar{h}, \bar{h}, \overline{l v}\right) & \operatorname{subcell}\left(\hat{z}_{1}+\bar{h}, \hat{z}_{2}+\bar{h}, \hat{z}_{3}+\bar{h}, \bar{h}, \overline{l v}\right)
\end{array}
$$

with $\bar{h}=h / 2$ and $\overline{l v}=$ level +1 .

Algorithm 1 exactly follows the 2D quadrangle sub-cell decomposition strategy applied for the composed integration of the cell integrals of 2.3 for plane problems and can analogously be

Preprint, submitted to Int. J. Numer. Meth. Engng. in 2012 
applied for the octree generation in 3D by replacing the subDivide() method corresponding to the geometrical needs.

\section{Numerical examples}

The main focus of the following numerical examples is on solving problems due to linear elasticity to reveal the performance of the weak boundary formulation applied to the finite cell method and to demonstrate the accuracy and reliability of the method, independent of fitted domain boundaries. Nevertheless, we document some basic characteristics of the afore introduced method using an illustrative 2D Laplace problem for which we also provide the Nitsche extension in compact form. A second benchmark problem includes curved boundaries with a focus on the accuracy of the method in terms of displacements and stresses. The performance of the method for non-homogeneous boundary conditions is demonstrated using a shear dominated benchmark problem that reveals FCM-specific requirements with regard to stabilization. A comparison of the proposed formulation with a classical finite element formulation is given for a thin plate bending example, to reveal the dependencies between the stabilization parameters and the overall accuracy of the method. Finally we demonstrate the versatility of the method and its usability in engineering practice and biomechanics using 3D problems. The last example is a classical isogeometric analysis of a trimmed shell that is used to demonstrate a conceptual strategy to overcome the trimming problem.

Throughout the following studies we will measure the convergence of an analysis in terms of the percentage of error in strain energy

$$
e_{E(\Omega)}=\left(\frac{|\mathcal{W}(u, u)-\mathcal{W}(\hat{u}, \hat{u})|}{|\mathcal{W}(u, u)|}\right)^{\frac{1}{2}} 100 \%
$$

where $u$ denotes the exact solution, $\hat{u}$ the finite cell solution and $\mathcal{W}(u, u)$ the total strain energy.

\subsection{D Laplace problem}

The following 2D Laplace problem is proposed in FERNÁNDEZ-MÉnDEZ ET AL. [22] to demonstrate the performance of weak boundary formulations for mesh-free methods. In the following we give a brief summary of the governing equations of the Laplace problem including the weak boundary formulation. A detailed description can be found in [22] or [20]. The geometry, boundary conditions and the analytical reference solution are provided in Fig. 10. Homogeneous Dirichlet boundary conditions and a sinusoidal boundary load along one of the edges of the square solution domain were applied.

Using the extensions introduced in section 3, the governing integral equation of the Laplace

Preprint, submitted to Int. J. Numer. Meth. Engng. in 2012 


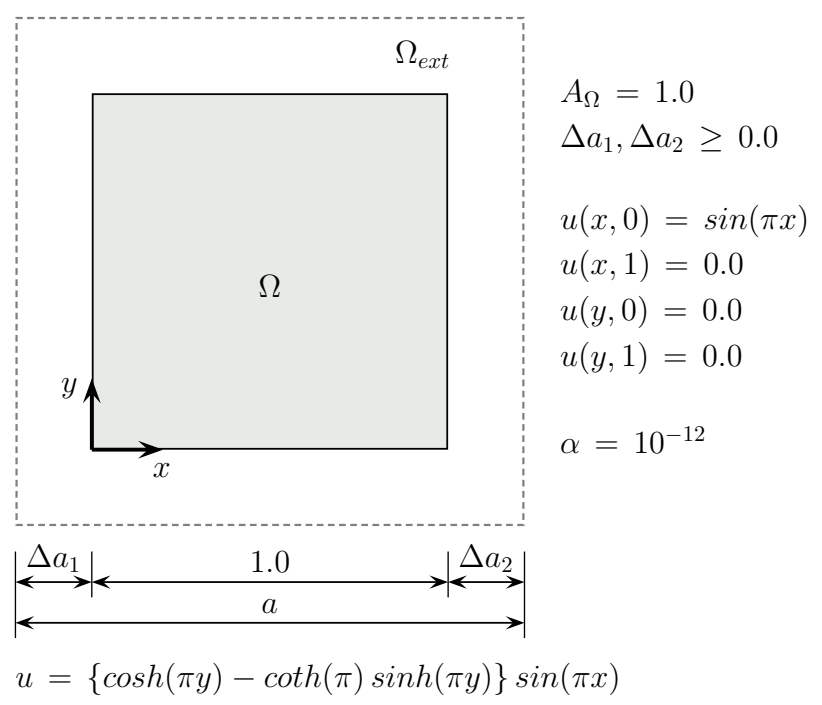

Figure 10: 2D Laplace problem.

problem formulated for the finite cell method reads

$$
\begin{array}{r}
\int_{\Omega_{\alpha}} \delta(\nabla u) \alpha(\nabla u)^{T} d a-\int_{\Gamma_{u}} \delta(\nabla u) \mathbf{n}^{T} u d r-\int_{\Gamma_{u}} \delta u(\nabla u \mathbf{n}) d r \\
+\int_{\Gamma_{u}} \tau \delta u u d r=\int_{\Gamma_{t}} \delta u t_{0} d r
\end{array}
$$

The primal value $u$ denotes a scalar function on $\Omega, \nabla u$ denotes a state vector on $\Omega$ and $t_{0}$ a boundary load along the domain edge $\Gamma_{t}$.

The characteristic solution of the Laplace problem is shown in Figure 11. A $8 \times 8$ fictitious domain grid was chosen such that the solution domain did not coincide with the cell grid to accommodate the independence of the method from the grid. In addition the shown problem configuration does not resolve fully the true domain $\Omega$ with integration sub-cells. Adaptive integration was performed for all examples to confine the integration error using a cell-wise quadtree generation. The applied tree depth was varied between $m=8$ and $m=12$ to disclose the influence of the integration level on the performance of the solution method. The extension domain was penalized with $\alpha=10^{-12}$ for the Laplace problem throughout all the computations. The stability parameter $\tau$ (34) was derived cell-wise from the solution of local eigenvalue problems in analogy to (31). Visualizations of the integration sub-cells were limited up to a quadtree level $m=4$.

The error distribution of the solution depicted in Fig. 11 confirms the results, reported in [22] and in particular, shows a reasonable error level along the fixed boundary at a polynomial degree of $p=3$.

A convergence study of the problem for various problem configurations in terms of the relative error in energy norm is shown Figure 12. The problem was solved for various extension domains

Preprint, submitted to Int. J. Numer. Meth. Engng. in 2012 


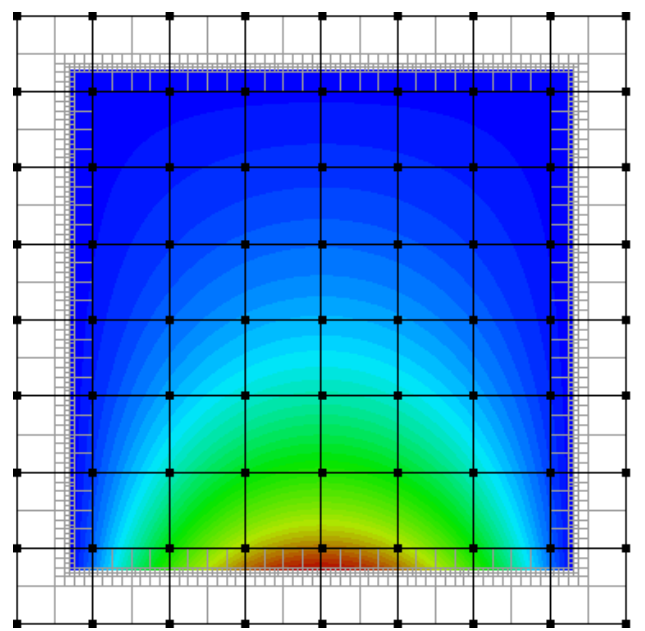

(a) FCM solution

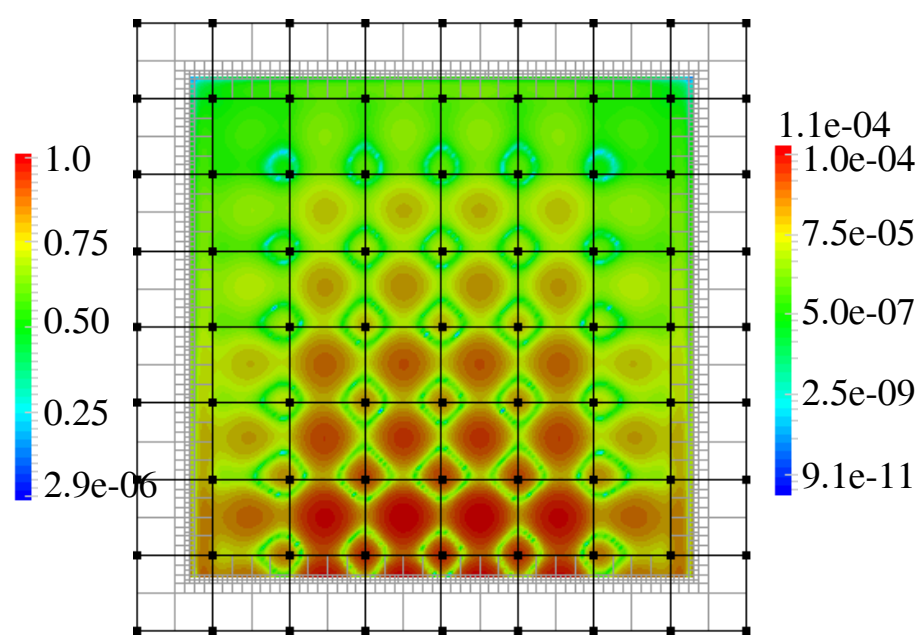

(b) absolute error

Figure 11: FCM solution and logarithmic plot of the error distribution for $\Omega$ embedded in $8 \times 8$ cells with $a=1.22$, polynomial degree $p=3$, FCM penalization $\alpha=1 . e-12$ and quadtree depth $m=8$.

and sub-cell resolutions. Strongly and weakly enforced boundary conditions (green curves) on a body-fitted cell grid were chosen as a reference solution for the problem. Both curves coincide up to a polynomial degree $p=6$ and deviate from their exponential convergence behavior where they meet the accuracy of the given reference energy value. The red and blue curves with outline triangles and squares, respectively, demonstrate the convergence behavior of the method when domain-fitted integration is assured by a corresponding sub-cell resolution. A rather large extension domain was chosen for the red curve such that the solution domain $\Omega$ was exactly integrated within $\Omega_{\alpha}$ by a single sub-cell layer in the boundary cells. The domain associated part of the boundary cells was triangulated (cf Fig. 13) for the blue curve to coincide with the domain boundary $\Gamma$. The exponential convergence behavior is lost for higher polynomial degrees due to the applied rather coarse triangle mesh. In principle the integration mesh can be chosen in analogy to the quadtree approach, cell-wise and independent of adjacent cells thus dramatically simplifying the mesh generation. In contrast to the quadtree approach the polynomial character of the integrand of (26) and (27) is lost due to the geometry mapping of the distorted integration triangles.

The extension domain with $a=1.22$ that was chosen for the remaining two curves representing the convergence of sub-cell unfitted boundaries (filled dots) is a severe test case for the method since even the finest applied sub-cell resolution (quadtree depth $m=12$ ) is not able exactly to cover the solution domain $\Omega$. Despite an acceptable error level below $1 \%$ in energy norm the convergence levels off for higher polynomial degrees clearly indicating the integration error. We applied Gauss quadrature with $(p+1)$ integration points per coordinate direction for each square sub-cell in all our computations. A corresponding number of integration points were chosen for the triangle sub-cell approach to guarantee the exact integration of a polynomial of the chosen 


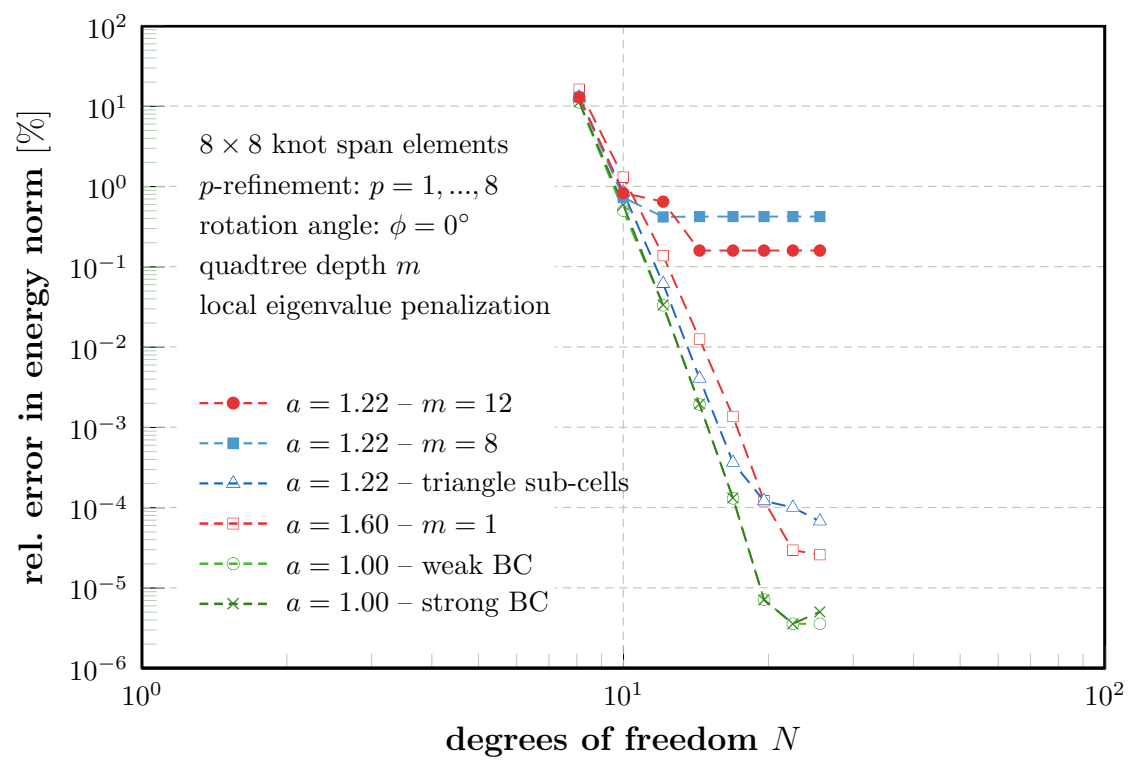

Figure 12: Convergence behavior of sub-cell fitted (outline dots) and non-fitted (filled dots) boundaries for uniform $p$-refinement of the unrotated $\left(\phi=0^{\circ}\right)$, centrically embedded domain $\Omega$.

polynomial degree.

The extensive independence of the solution quality from the chosen cell orientation is illustrated in Figure 13 in which the logarithmic error distribution for a rotated solution domain $\Omega$ within $\Omega_{\alpha}$ is shown. A rotation by $\phi=9^{\circ}$ and $\phi=23^{\circ}$ was chosen arbitrarily to account for the stability of the method. The extension domain was adjusted to ensure a completely embedded domain $\Omega$. Despite a relatively large number of cut boundary cells with a very small contribution to $\Omega$ the maximum absolute error in the solution domain was almost kept constant at a level of $<0.05 \%$. The convergence study for uniform $p$-refinement (Figure 14) showed that the rotated domain profits from the irregular distribution of the quadrature points, resulting in a smaller energy norm error of at least one order of magnitude for the various quadtree depths compared to the unrotated case (Fig 12). The curve for the triangle sub-cell integration is comparable to the unrotated case and mainly influenced by the coarse triangle mesh and a partially distorted triangle geometry, cf Fig. 15.

Looking at the solution and error plot given in Figure 15 we can see the principal behavior of the finite cell method beyond the physical domain boundary. From the loaded edge along $\Gamma_{t}$ in Figure 15-(a) it can be seen that the solution smoothly extends into the fictitious domain. The interpolation space that is constraint over the solution domain dissipates with arbitrary oscillations outside $\Omega$. Any additional constraint outside $\Omega$ destroys this behavior with a direct and negative effect on the solution. Similarly Figure 15-(b) shows that the amplitudes of this oscillatory behavior are orders of magnitude higher in the extension domain than in $\Omega$ this way forcing the multi-variate B-splines to obey the solution within $\Omega$.

The evolution of the stability parameter for uniform $p$-refinement derived from a local and global

Preprint, submitted to Int. J. Numer. Meth. Engng. in 2012 


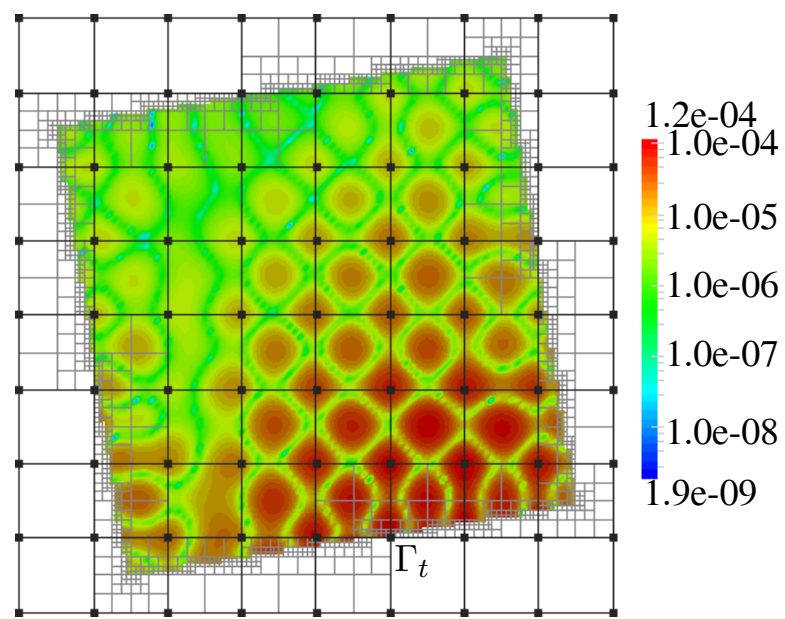

(a) $\phi=9^{\circ}, a=1.3$

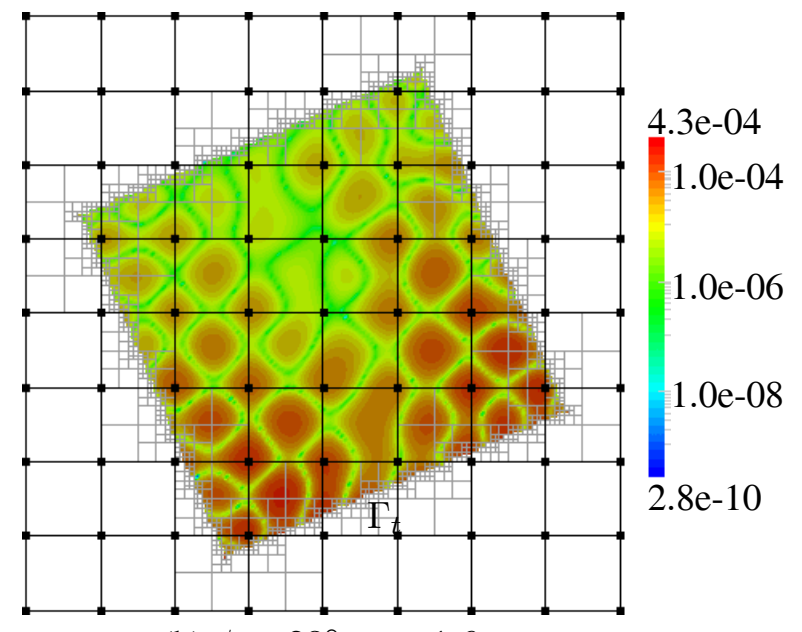

(b) $\phi=23^{\circ}, a=1.6$

Figure 13: Logarithmic plot of the absolute error distribution for $p=3$, FCM penalization $\alpha=1 . e-12$ and quadtree depth $m=8$.

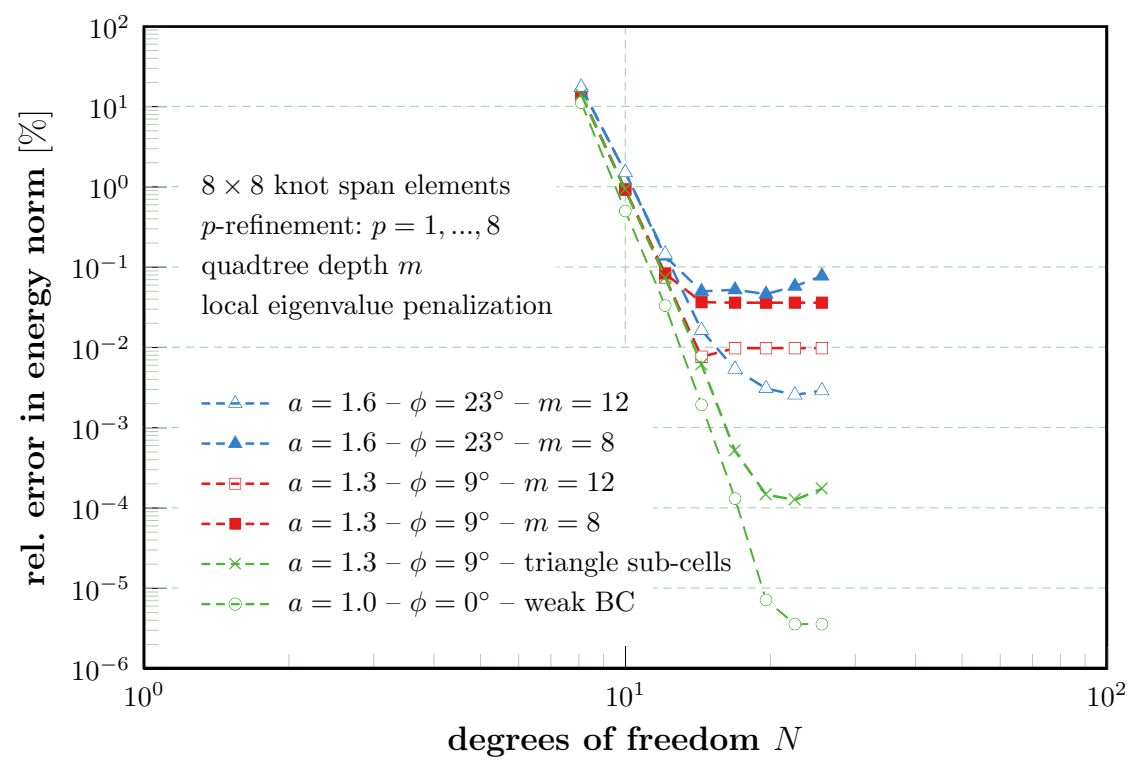

Figure 14: Convergence behavior of the rotated domain $\Omega$ for uniform $p$-refinement.

Preprint, submitted to Int. J. Numer. Meth. Engng. in 2012 


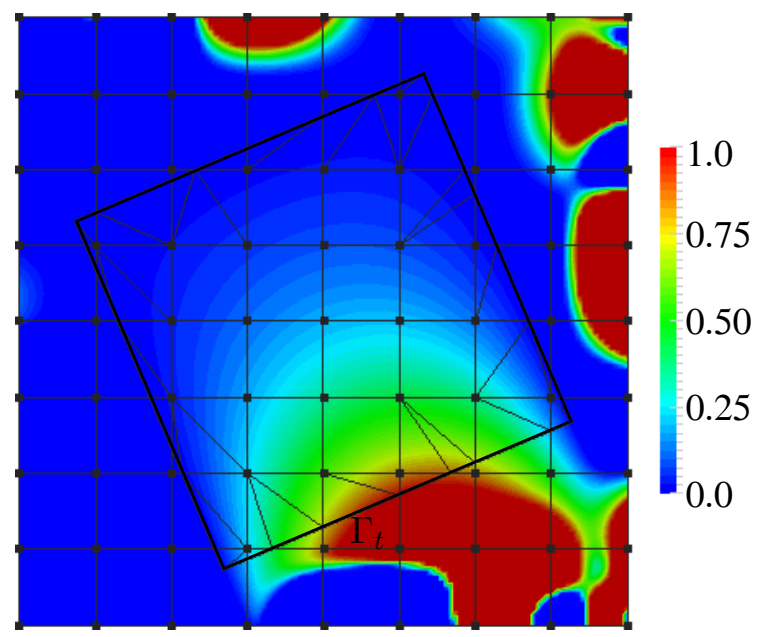

(a) $\phi=23^{\circ}$

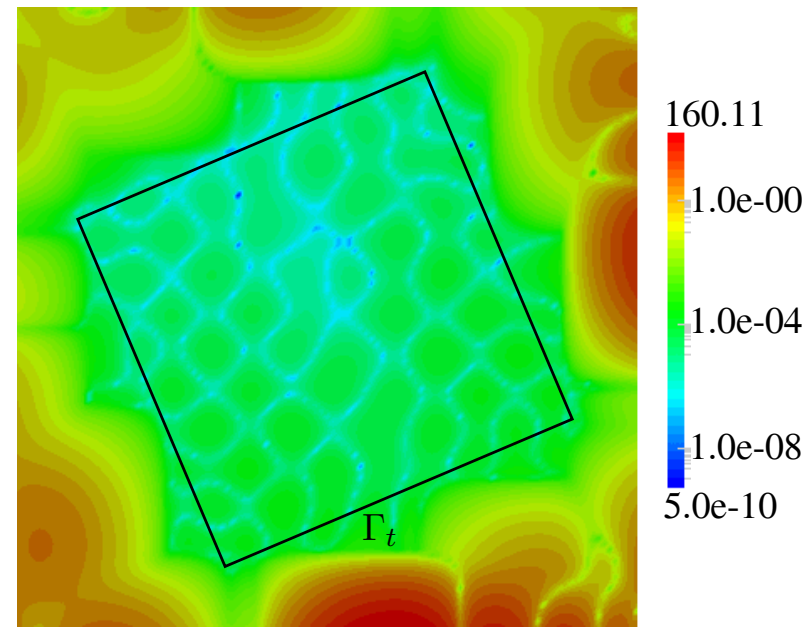

(b) $\phi=23^{\circ}$

Figure 15: Solution from triangle sub-cell integration and logarithmic error plot $(m=8)$, both including the fictitious extension domain for $p=3, a=1.60$ and FCM penalization $\alpha=1 . e-12$.

eigenvalue analysis is shown in Figure 16. The green curves refer to the $23^{\circ}$ rotated problem (Fig. 13), the blue curves refer to the unrotated problem (Fig. 11). The reference curve for the unrotated, domain fitted problem is shown in red. The largest (filled dots) and smallest (open dots) maximum eigenvalue that was found in the set of boundary cells for each of the unfitted problems is reported, in addition to the globally derived stability parameter $\max \lambda$. The regularity and symmetry of the unrotated problem is very well reflected in the three corresponding blue curves that congruently evolve up to $p=4$ and form a relatively tight and compact hull for higher degrees. The closely spaced curves confirm the similar contributions of the penalty terms of the cut boundary elements to the solution domain. The global and local stability parameters are in good agreement and will not dominate the accuracy of the solution in any part of the domain $\Omega$ (cf Fig. 11). In contrast, the green curves for the rotated problem already spread significantly for lower polynomial degrees, clearly indicating the various contributions of the cut boundary cells to the solution domain. The degenerated boundary cells with a very small domain contribution clearly dominate the stability parameter distribution. Despite the significantly higher values obtained from the local approach compared to the global parameters this strategy is favored since only a few cells are penalized along their boundary with a large value whereas the global approach penalizes each boundary cell.

\subsection{Plane stress annular plate}

A plane stress ring plate is modeled to account for the performance of the method for problems due to linear elasticity as presented in section 3 . The analytically described ring geometry was embedded in a simulation domain grid consisting of $8 \times 8$ knot span elements (cf Fig. 19(a)). An exact parametric description of the circular boundary was chosen to reduce the modelling error of the problem. Homogeneous Dirichlet boundary conditions were prescribed along the outer 


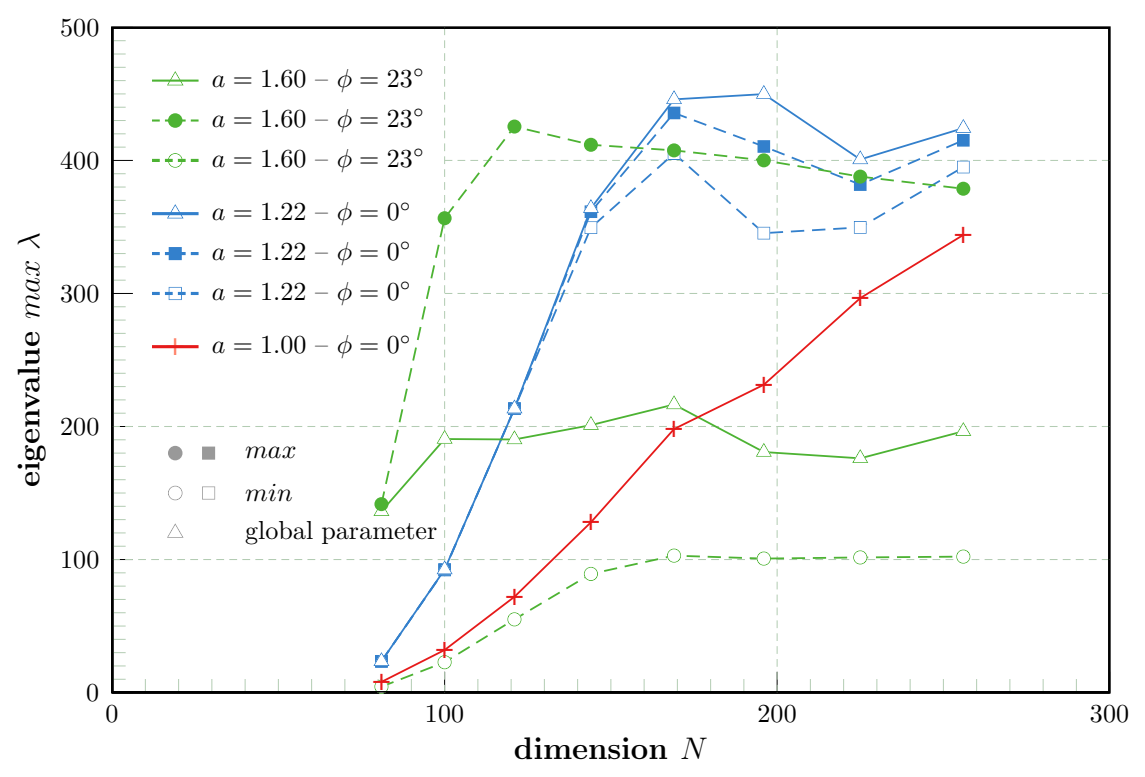

Figure 16: Stability parameter $\tau(\max \lambda)$ for uniform $p$-refinement.

radius $r_{o}$ and a prescribed constant radial pressure force $\mathbf{t}_{0}$ was applied along the inner radius $r_{i}$. In addition the plate domain was loaded with a in radial direction exponentially decreasing area load $\mathbf{p}_{0}$. The geometry, loading, boundary conditions and the analytical reference solution of the displacements field and the state of stress in polar coordinates $(r, \theta)$ is given in Fig. 17. The stability parameter $\tau_{N}$ vanishes with a Poisson ratio of $\nu=0$ thus simplifying an optimal choice for the remaining stability parameter $\tau_{S}$.

Various knot span element discretizations were chosen for a convergence study with uniform p-refinement. The smooth convergence behavior for each model with a slight tendency to exponential rates, resulting in below $1 \%$ relative error in energy norm for a $(8 \times 8)$-knot span element discretization is shown in Figure 18. In contrast to the p-version of the finite cell method [49] in which high-order hexahedral cells are applied, the B-spline version requires a larger number of knot span elements to ensure the expected convergence behavior. Due to the large number of shared spline functions between adjacent knot spans particularly for higher polynomial degrees the numerical effort in term of degrees of freedom and bandwidth characteristics remains essentially unchanged. The need for a sufficient mesh density is a characteristic of the B-spline version that has been found before in $[49,42]$ for various examples and that can be observed also in Figure 18. The acceptable but moderate convergence progress for $(4 \times 4)$ and $(8 \times 8)$-knot span elements abruptly jumps down for the $(16 \times 16)$ discretization to an error level that has improved by at least one order of magnitude.

The stability parameter for the convergence study were found locally. Due to the symmetry of the problem and the symmetric model a global choice also gives reasonable results, as shown in Figures 21 and 22.

An overall impression of the displacement field solution is given in Figure 19-(a). Even for lower 


$$
\begin{array}{lr}
r_{o}=1.0 & \\
r_{i}=0.25 & \forall \mathbf{x} \in \Gamma_{o} \\
\mathbf{u}=\mathbf{0} & \\
\mathbf{t}_{0}=\left(\frac{1}{2} \frac{\ln 0.25+1}{\ln 2}\right) \mathbf{n} & \forall \mathbf{x} \in \Gamma_{i} \\
\mathbf{p}_{0}=\left(\frac{1}{r \ln 2}\right) \mathbf{n} & \forall \mathbf{x} \in \Omega \\
E & =1.0 \\
\nu & =0.0 \\
\alpha & =10^{-12}
\end{array}
$$

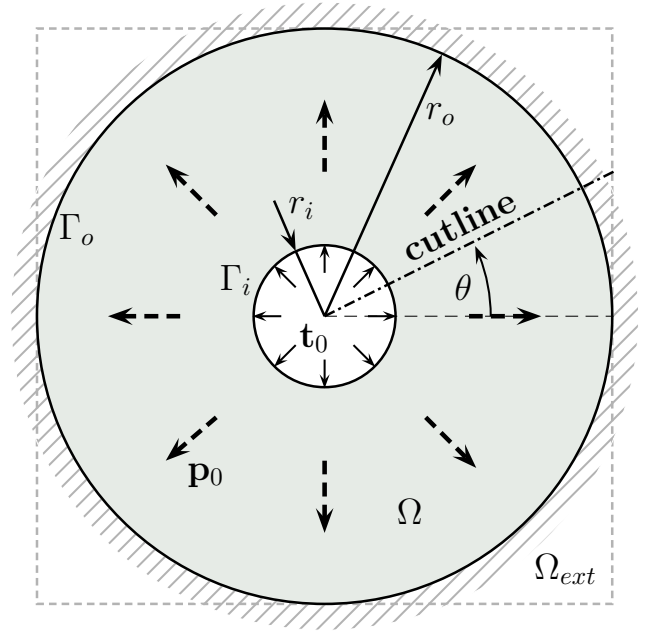

displacement field

$\mathbf{u}_{r}=-\frac{r}{2} \frac{\ln r}{\ln 2}$

$\mathbf{u}_{\theta}=0.0$

state of stress

$\sigma_{r}=-\frac{1}{2} \frac{\ln r+1}{\ln 2}$

$\sigma_{\theta}=-\frac{1}{2} \frac{\ln r}{\ln 2}$

$\sigma_{r \theta}=0.0$

Figure 17: 2D ring plate problem.

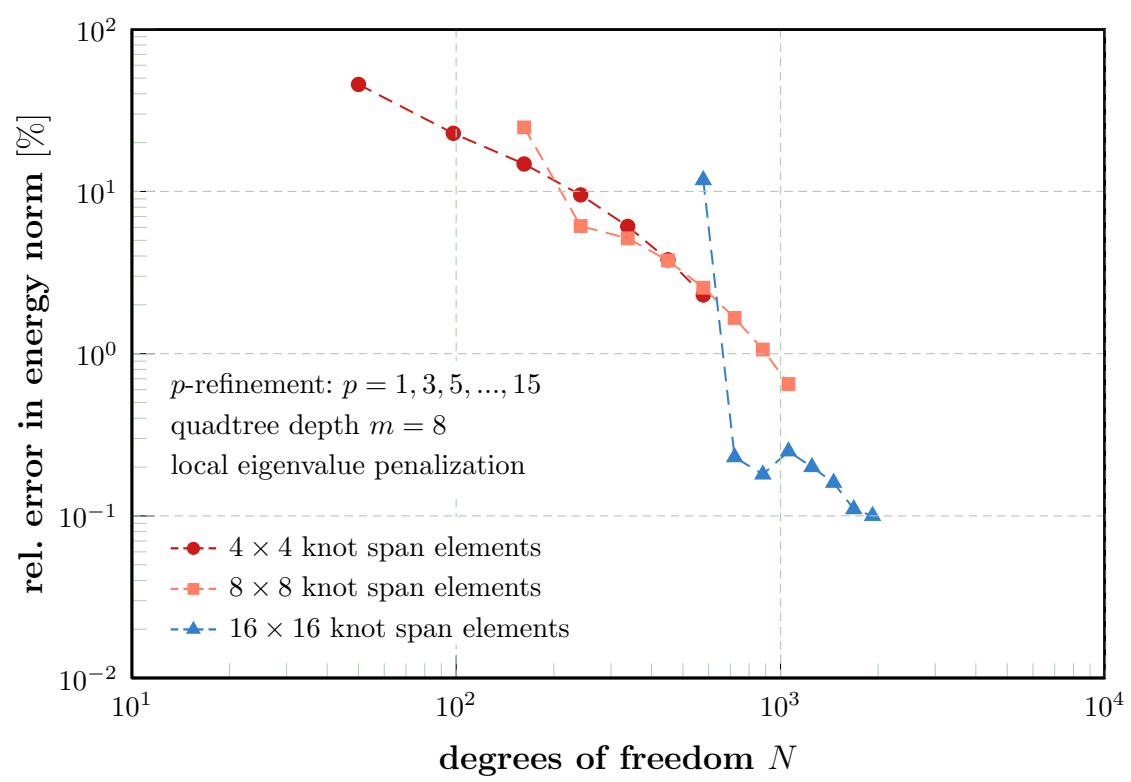

Figure 18: Convergence behavior for uniform $p$-refinement.

Preprint, submitted to Int. J. Numer. Meth. Engng. in 2012 


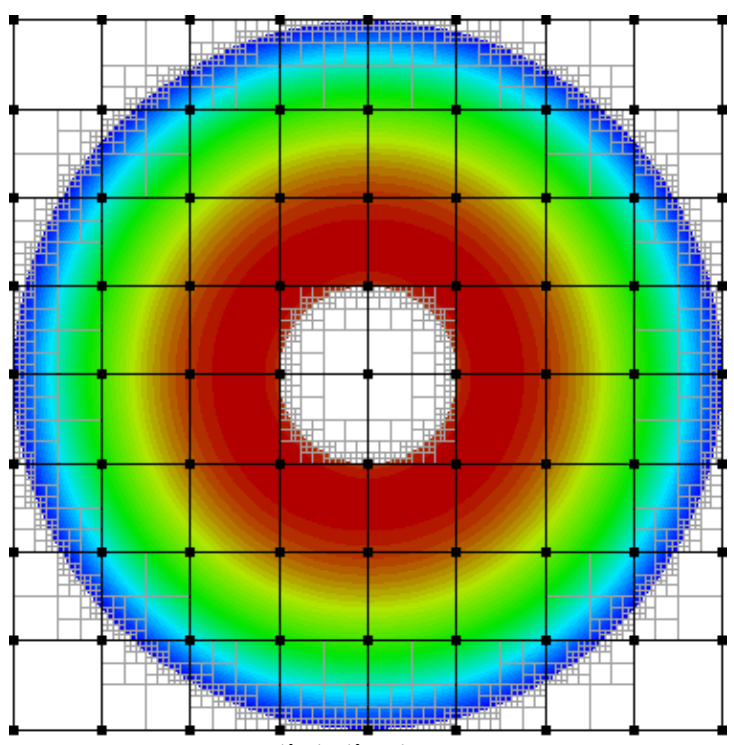

(a) radial displacement

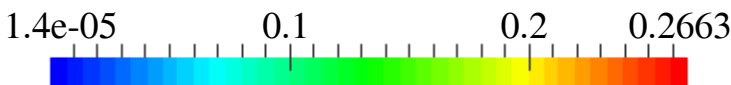

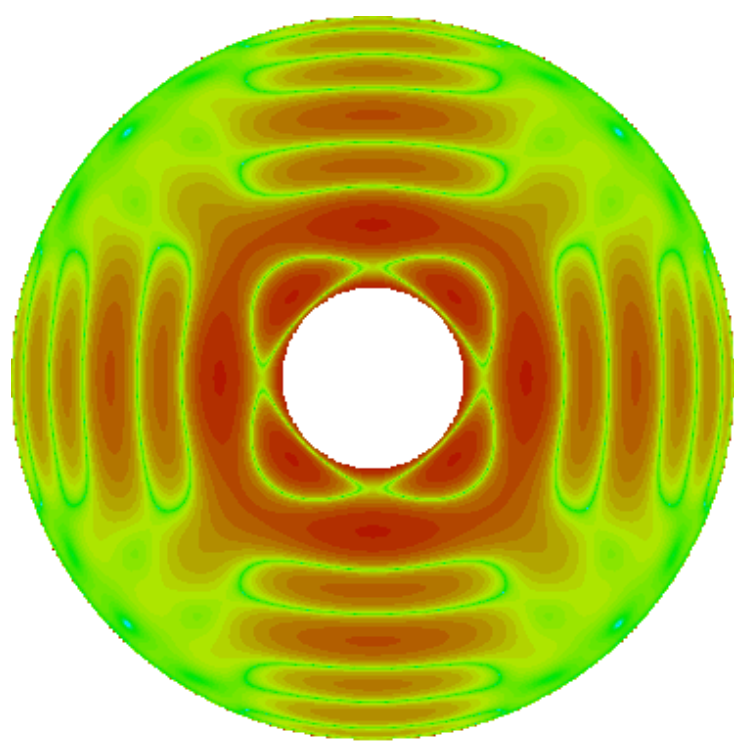

(b) absolute error

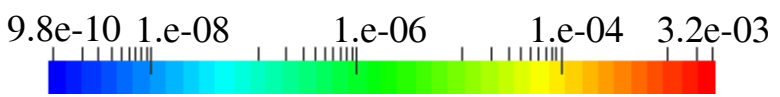

Figure 19: Solution and logarithmic error plot of the displacement field for $p=8$ and $m=8$.

polynomial degrees the depicted smoothness of the displacement field is observed at similar accuracy levels. A logarithmic error plot of the absolute error distribution is shown in Figure 19-(b). In particular along the outer radius of the ring plate, where homogeneous boundary conditions are weakly enforced, a satisfying result can be noticed without any identifiable negative effect from the boundary penalization. The complete symmetry in the error distribution also indicates the high stability of the proposed method.

Similarly, a good quality of the solution is found for the von Mises stresses and corresponding error distribution depicted in Figure 20. The maximum error in the stresses is found along the inner radius. The elements embedding the inner void domain $\hat{\Omega}_{\alpha}\left(r<r_{i}\right)$ mutually influence a smooth extension of the stresses into $\hat{\Omega}_{e x t}$ (cf Fig. 20-(b)) thus introducing a constraint that reflects the maximum error along the inner radius. The very good agreement of the predicted displacements and stresses with the analytic solution is presented in Figures 21 and 22, respectively. The diagrams show pointwise results along a $26^{\circ}$ inclined cutting line from the center to the boundary of the extension domain $\Omega_{e x t}$ (cf Fig. 17). The displacements were found to be identical within $\Omega$ even for the lower polynomial degrees. The von Mises stresses (Fig. 22) also show a very accurate agreement with the reference solution with minimal deviations at the inner boundary due to the aforementioned artificial symmetry induced constraints. 
M. Ruess et al. - Weak Boundary Conditions for the FCM

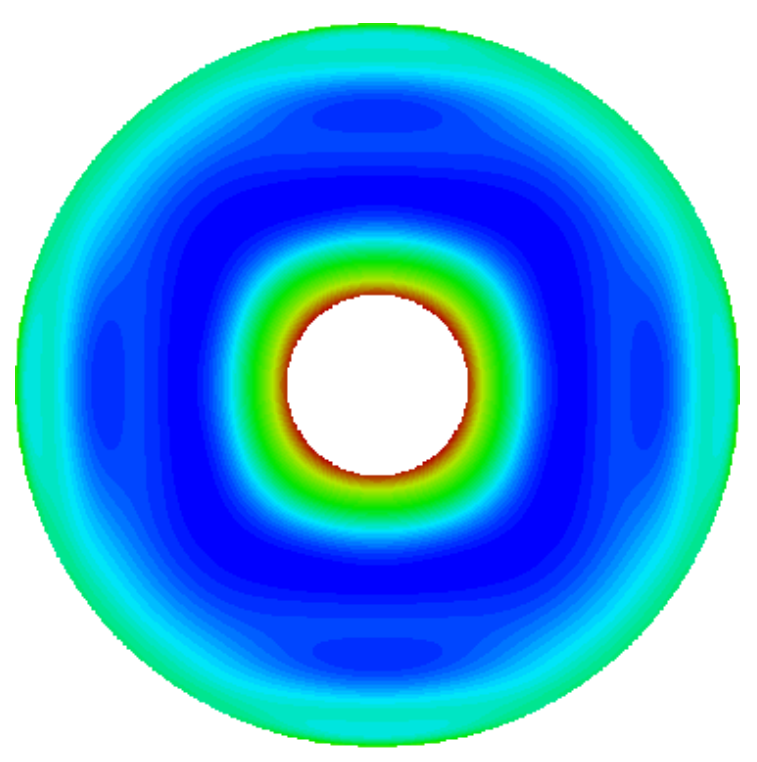

(a) von Mises stress

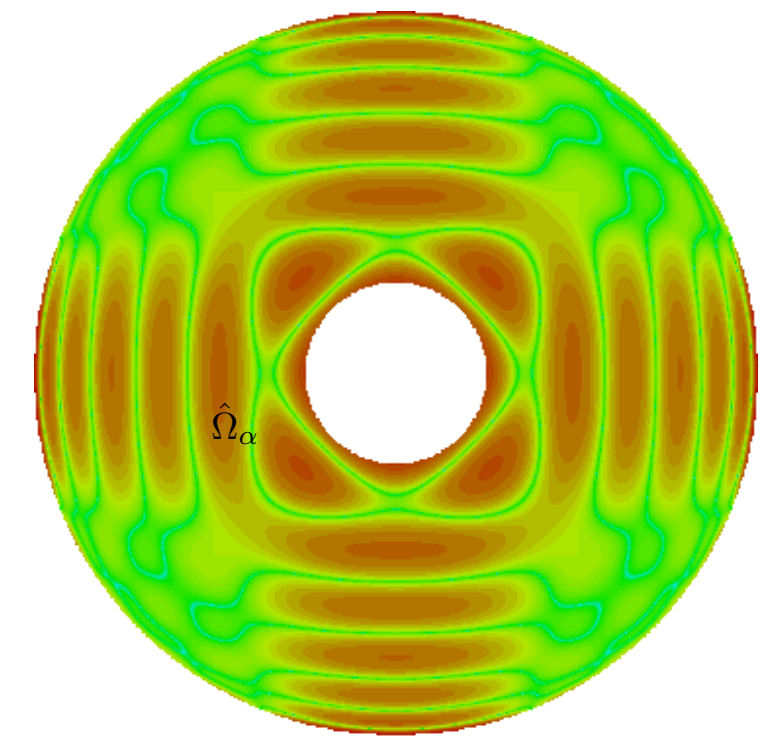

(b) absolute error

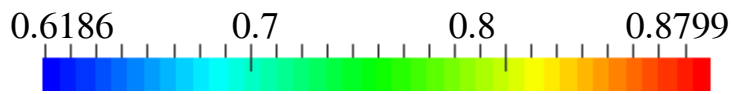
2.5e-07 1.e-05 1.e-04 1 .e-03 $1 . \mathrm{e}-02 \quad 6.7 \mathrm{e}-02$

Figure 20: Solution and logarithmic error plot of the von Mises stress distribution for $p=8$ and $m=8$.

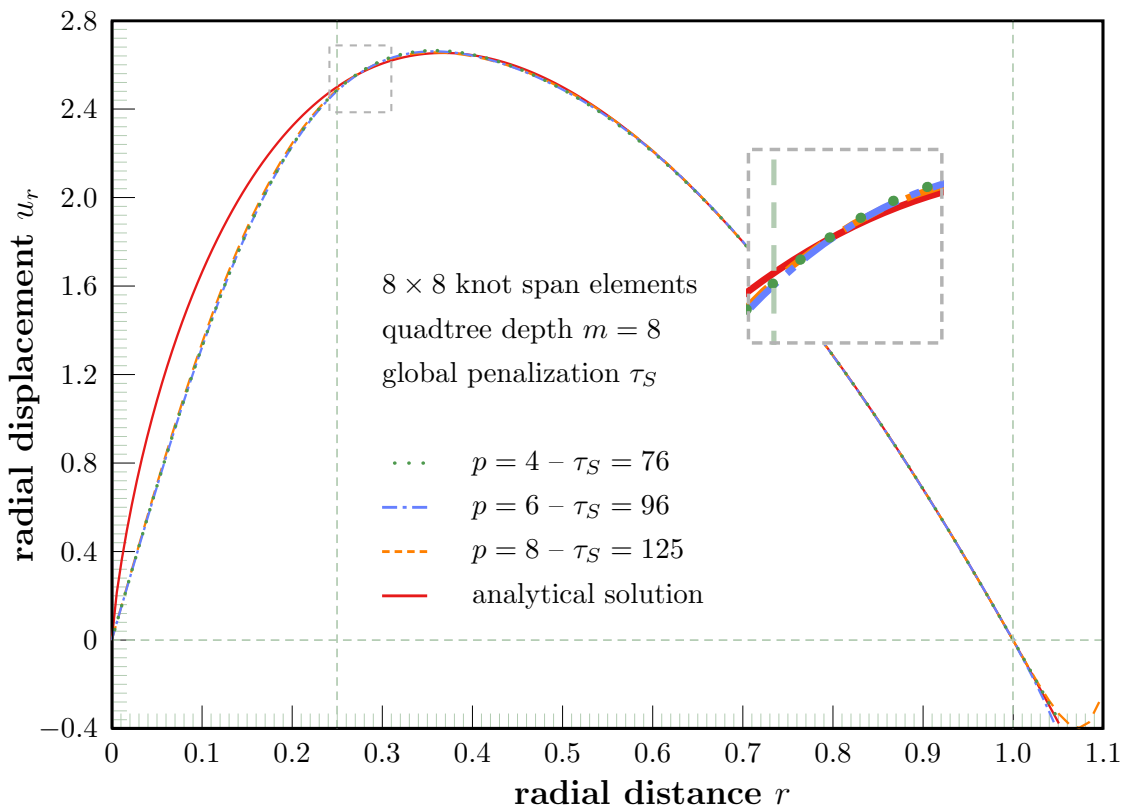

Figure 21: Displacement along a $26^{\circ}$ inclined cutline.

Preprint, submitted to Int. J. Numer. Meth. Engng. in 2012 
M. Ruess et al. - Weak Boundary Conditions for the FCM

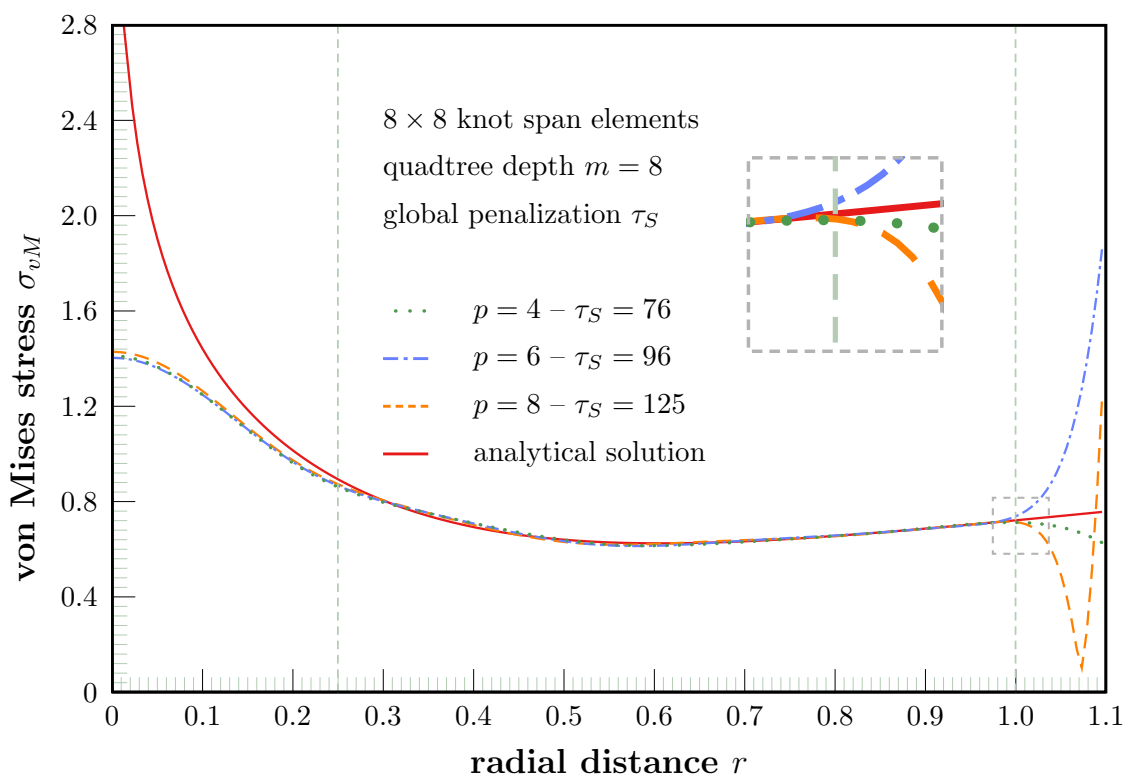

Figure 22: Von Mises stress distribution along a $26^{\circ}$ inclined cutline.

\subsection{Curved beam subjected to end shear}

$$
\begin{aligned}
& r_{o}=10.0 \\
& r_{i}=5.0 \\
& u_{0}=0.01 \quad \forall \mathbf{x} \in \Gamma_{I I} \\
& u_{1}=0 \quad \forall \mathbf{x} \in \Gamma_{I} \\
& u_{2}=0 \quad \forall \mathbf{x} \in \Gamma_{I} \wedge x_{2}=r_{i} \\
& E=10,000 \\
& \nu=0.25 \\
& \alpha=10^{-14}
\end{aligned}
$$

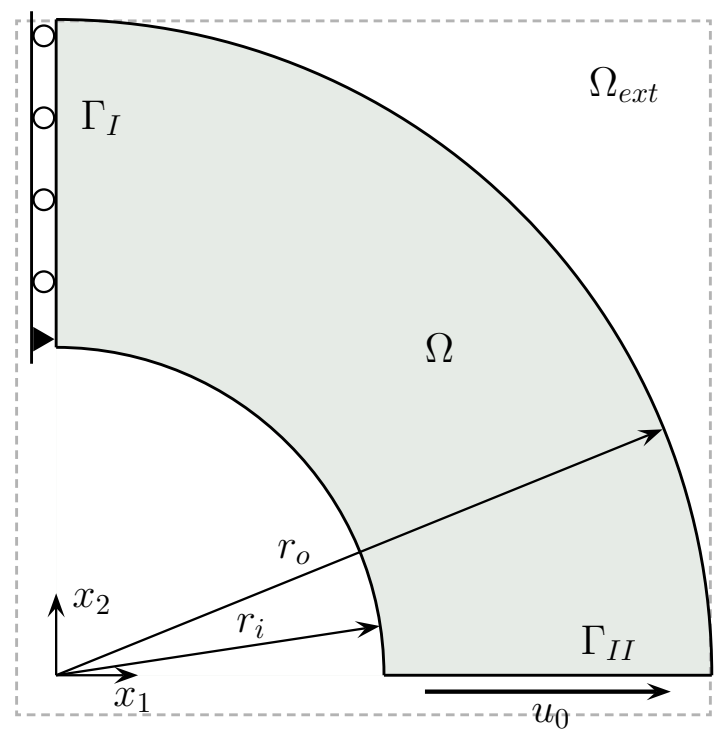

Figure 23: Curved beam problem under shear deformation.

The following example of a curved beam subjected to an end shear load was chosen to account for the weak enforcement of non-homogeneous boundary conditions. The geometry and the model 
parameters of this example are illustrated in Figure 23. The extension domain $\Omega_{\text {ext }}$ was chosen such that the boundary $\Gamma_{u}$ was completely embedded and does not coincide with the boundary $\partial \Omega_{\alpha}$ of the embedding domain. A constant prescribed displacement of $u_{0}=0.01$ units was subjected to the lower beam boundary $\Gamma_{I I}$. Homogeneous boundary conditions were applied to $\Gamma_{I}$ at $x_{2}=r_{i}$. All other points along $\Gamma_{I}$ moved freely in the $x_{2}$-direction. A plane stress reference solution can be found in [59]. The stabilization values $\tau_{S}$ and $\tau_{N}$ for this problem were found as the eigenvalues of largest magnitude $\left|\lambda_{\max }\right|$ from the local eigenvalue problem (30) times a constant factor $\hat{C}_{(S, N)}$. In $[25,20]$ et al., a constant of not much larger than 2 for $\hat{C}_{(S, N)}$ is proposed which also ensured positive definiteness of the system stiffness matrix in all our computations. However, for some problems we realized a significant improvement of the accuracy of our results using higher values for $\hat{C}_{(S, N)}$ which was due to the fictitious domain approach that considers cells with arbitrarily small contributions to the physical domain $\Omega$ and was also due to the finite accuracy of the sub-cell integration scheme. We think that larger values of $\mathcal{O}(1)$ for $\hat{C}_{(S, N)}$ still preserve the basic character of the method without degeneration to a pure penalty method. The following analysis results supports our choice of larger stability values.
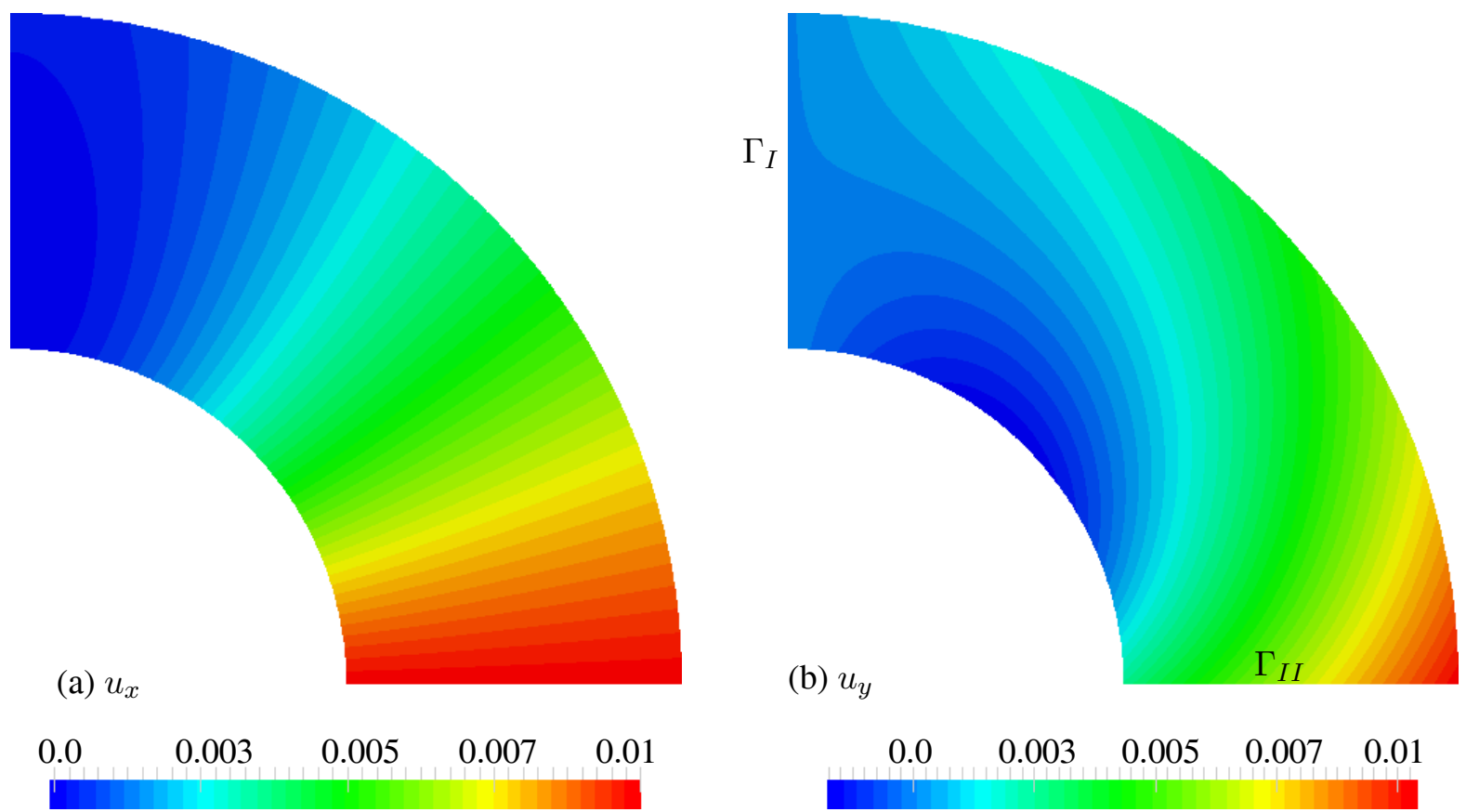

Figure 24: Displacement field in $\mathrm{x}$-direction (left) and in $\mathrm{y}$-direction (right) for $16 \times 16$-knot span elements.

The displacement field in $x$ - and $y$-direction of the curved beam is shown in Figure 24. A quadtree depth of $m=8$ was chosen for the sub-cell integration that includes all edges of the beam. The overall absolute displacement error was below $0.2 \%$. In particular along the 

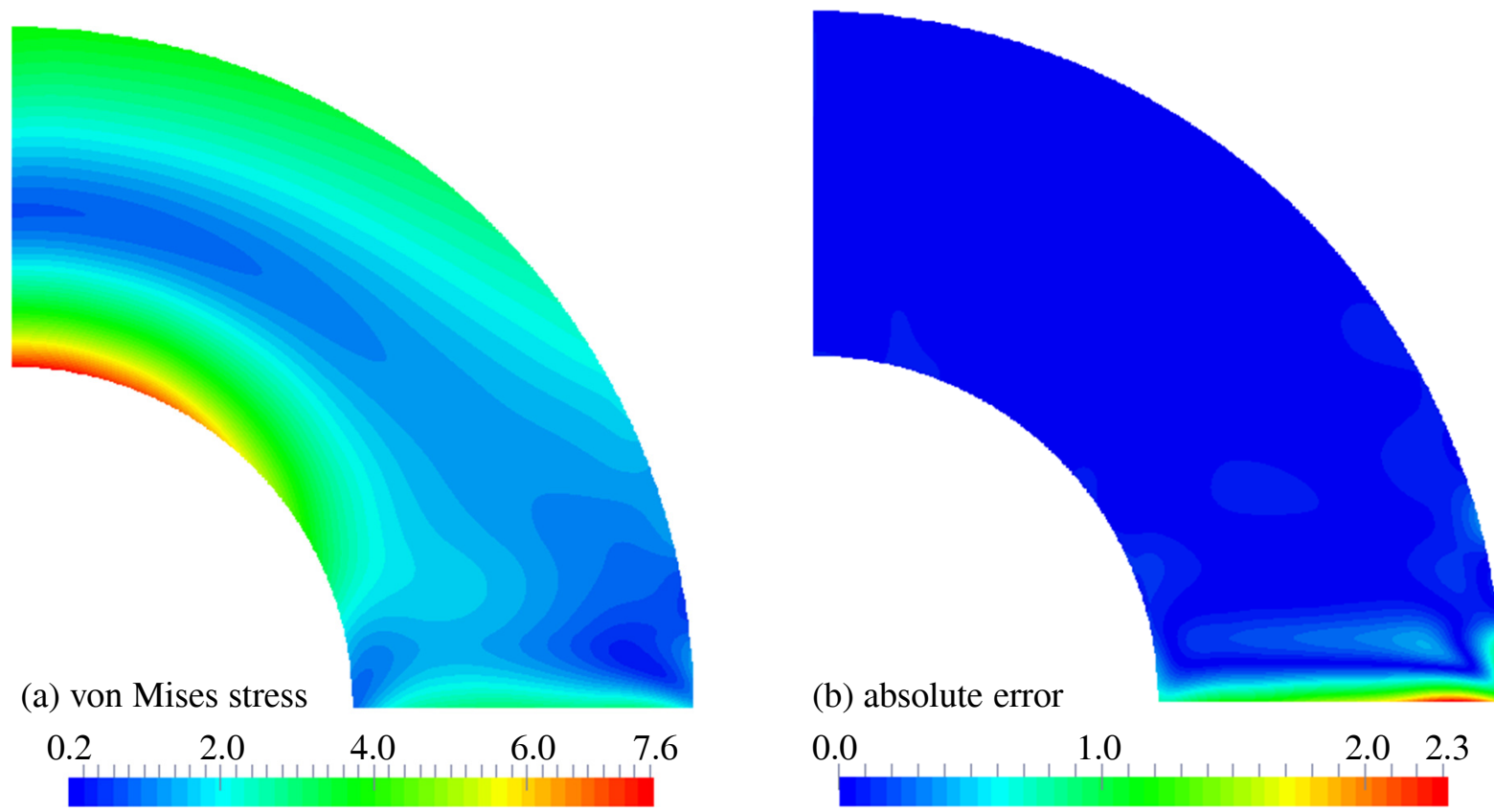

Figure 25: Von Mises stress distribution and error plot of the von Mises stress distribution for insufficient stabilization along $\Gamma_{I I}$.

boundary $\Gamma_{I}$ the error was in the range of the machine precision. At $\Gamma_{I I}$ a locally confined error concentration of below $0.4 \%$ was observed for $u_{y}$ at the lower right corner of the beam.

The $16 \times 16$-knot span elements discretization of the simulation domain resulted in a mesh size parameter $h=0.625$. The maximum eigenvalues of the cell-wise evaluation of (30) range corresponded to the polynomial degree $(p=3)$ and the cell contribution to the physical domain $\Omega$ in the interval $[12,24]$. A choice of $C_{(S, N)}=4 \max \lambda_{(S, N)}$, respectively, for the cut boundary cells provided an error level of $e_{E_{\Omega}} \approx 10 \%$ (error in energy norm). The source of this poor accuracy was readily seen in the von Mises stresses and was attributable to insufficient stabilization along the inhomogeneous boundary $\Gamma_{I I}$ (Fig. 25). An increase of $\hat{C}_{S}$ only for the cells along $\Gamma_{I I}$ by less than one order of magnitude removes this error completely and provides the expected accuracy level (Fig. 26).

The error spots in Fig. 26(b) reflect the shape of the derivatives of the underlying cubic B-spline. This behavior smoothens out for higher polynomial degrees and nearly vanishes due to the higher order continuity of the applied Ansatz. The displacement field and corresponding error did not noticeably profit from the increased stabilization. A further increase of $\hat{C}_{S}$ did not improve any of the results but started to deteriorate the stress distribution for excessive values, thus, clearly indicating the character of the method compared to a pure penalty approach.

The error in energy norm at $p=3$ is $e_{E_{\Omega}}=1.5 \%$ for the sufficiently stabilized model and easily drops down below $1 \%$ for higher p-degrees, e.g. for $p=5$ we find a relative error of $e_{E_{\Omega}}=0.5 \%$. In comparison to classical boundary fitted Lagrange-type finite elements the results are equivalent 

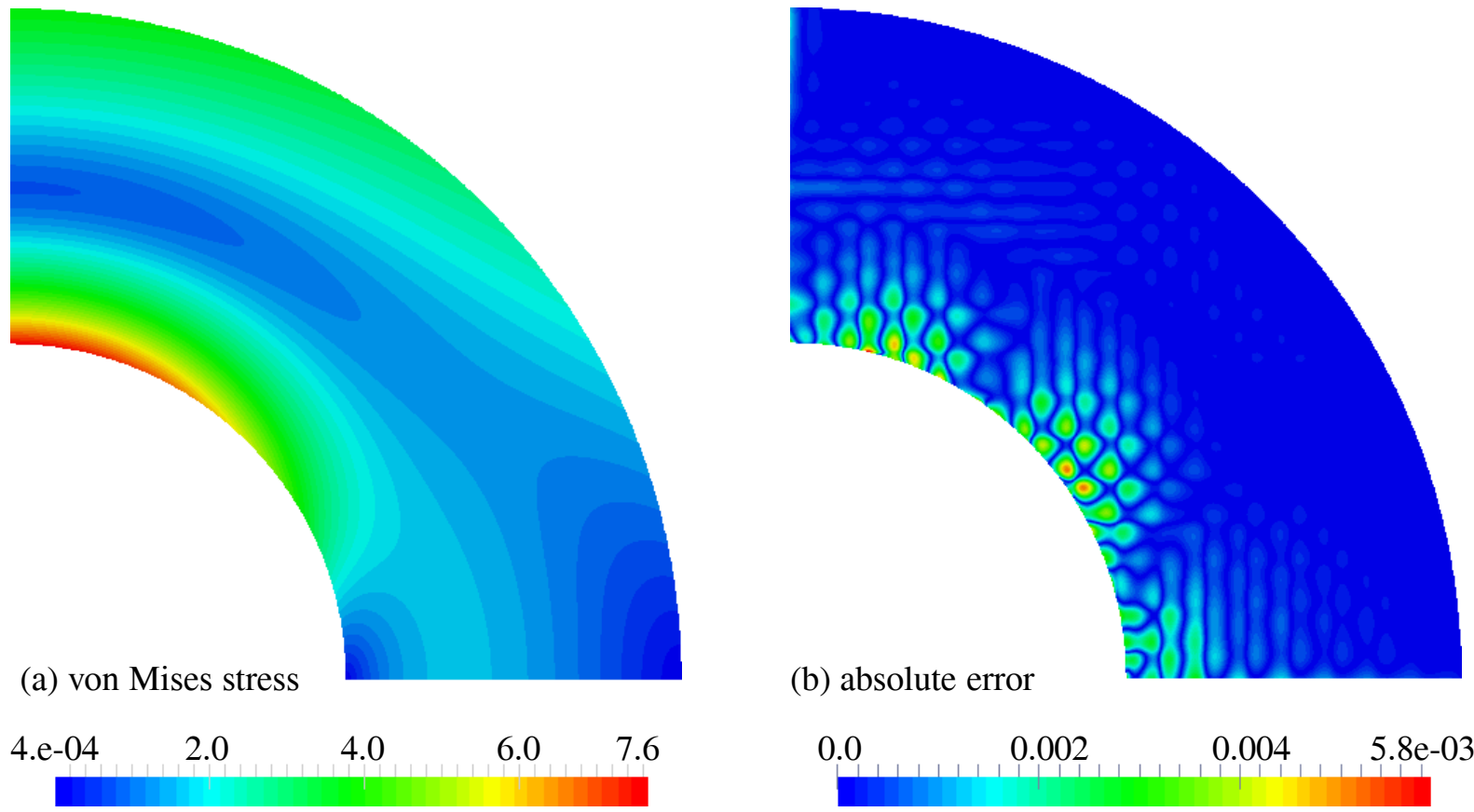

Figure 26: von Mises stress distribution and error plot of the von Mises stress distribution.

to bi-quadratic and bi-cubic elements, respectively (cf. [59]), at a comparable number of degrees of freedom.

\subsection{Plate bending: square and circular domain}

In this example the proposed method for a plate bending problem was compared with results from a thin plate finite element analysis. The FCM-plate structure was modeled as a 3D solid and eccentrically embedded in a fictitious domain such that the applied octree depth could not fully resolve the in-plane plate geometry with integration sub-cells. The geometry and material parameters of the model are given in Figure 27. The plate boundary was fully clamped. An aspect ratio of 100 well-justifies a comparison with a thin plate according to the theory of KirchhoffLove [6]. With the chosen load distribution the analytic solution of the Kirchhoff-Love model for the center deflection was independent of the Poisson ratio $\nu$ whereas the bending moment strongly depended on $\nu$. In the following a Poisson ratio of $\nu=0.3$ was chosen to account for the contribution of both stability parameter of the weak boundary formulation, $\tau_{N}$ and $\tau_{S}$ and the corresponding constants $C_{N}$ and $C_{S}$. Optimal values for $C_{N}$ and $C_{S}$ were found experimentally with $C_{N}=32$ and $C_{S}=32$ resulting in a relative error of the center deflection of below $1 \%$ on a $16 \times 16$ element grid for both models. A study on optimal values for the stability parameter $C_{S}$ for different Poisson ratios and various dimensions of the embedding domain, indicated by the domain extension value $\Delta$ in both coordinate directions is shown in Figure 28. The curves start with values for which the ellipticity of the problem is ensured. The stability value $C_{N}$ vanishes

Preprint, submitted to Int. J. Numer. Meth. Engng. in 2012 


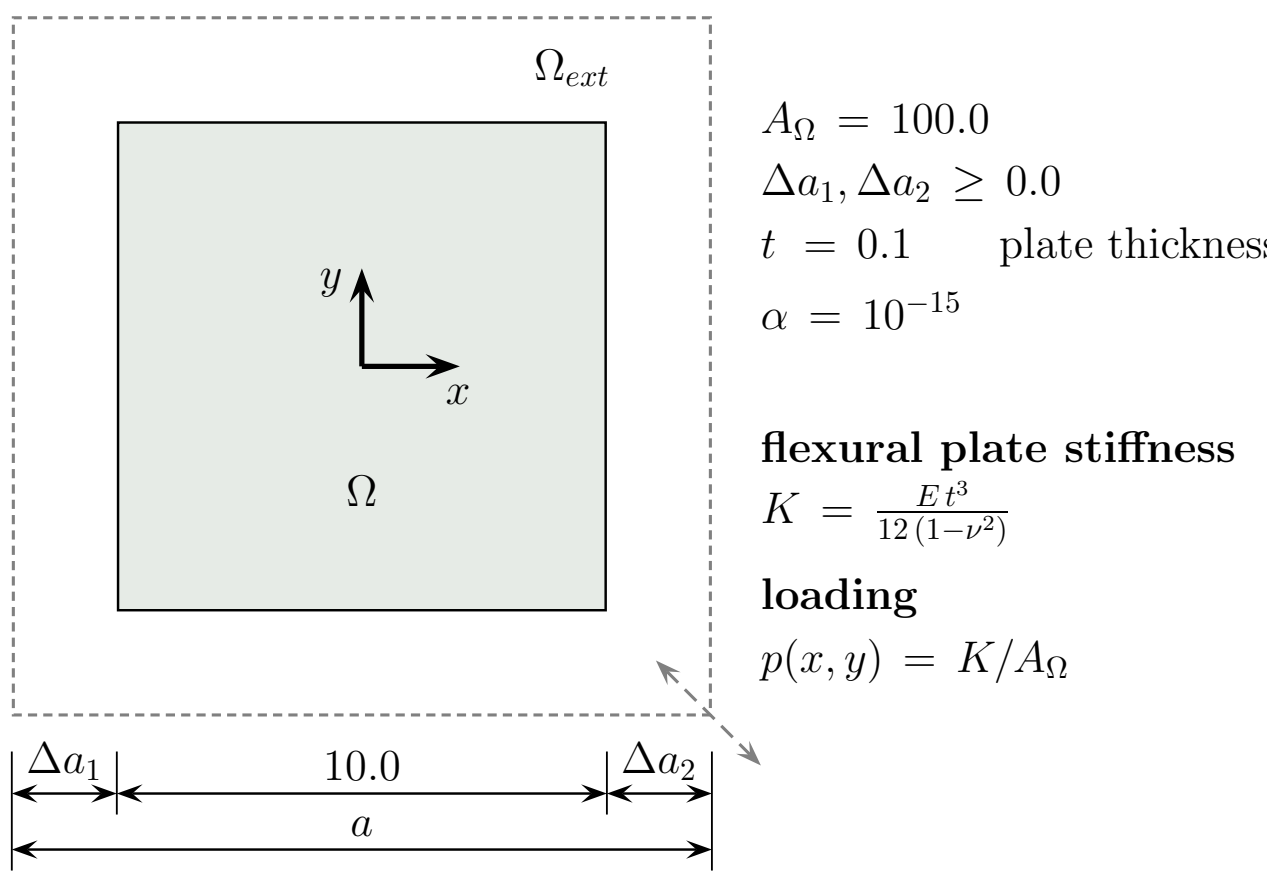

Figure 27: Square plate - model parameters.

(dashed curves) for a Poisson ratio $\nu=0$ (cf. eq. (22)). In this case, the optimal values for $C_{S}$ slightly decrease with an increase of the fictitious extension domain. This behavior could not be uniquely observed for $\nu \neq 0$ when, in addition, the influence of $C_{N}$ had to be taken into consideration. A reliably stable value for $C_{N}$ was derived from an eigenvalue analysis on cell level. Since the largest eigenvalue of this analysis was considered only as a reference value for a lower bound of $C_{N}$, optimality could not be assured. The steep gradient in the vicinity of the minimal energy norm error indicated a high sensitivity for an optimal choice of $C_{S}$ and $C_{N}$. Hence the peaks in the curves in Fig. 28 should be regarded as a principal region for optimal values rather than their exact location. Nevertheless good accuracy in displacements and stresses was also observed for a wide range of values beyond the optimality peak making the right choice less critical than indicated by the curves shown in Fig. 28. It is worth mentioning that the error level $\left(e_{E_{\Omega}} \approx 10 \%\right)$ for the embedded square plate, discretized with $(8 \times 8)$ knot-span elements at $p=3$ corresponds very well to the boundary fitted finite element solution according to the Kirchhoff-Love theory, discretized with a regular triangle mesh on a $(8 \times 8)$ grid and cubic Hermite polynomials. A qualitative comparison of the moment stress resultants $\mathbf{m}_{11}$ and $\mathbf{m}_{12}$ referring to the plate's mid-plane showed virtually no difference in the stress distribution (Figs. 29,30). A quantitative comparison revealed a relative difference in the extreme values of $0.36 \%-0.48 \%$ for $\mathbf{m}_{11}$ and a relative difference of $(-2.93)-(-3.66) \%$ for $\mathbf{m}_{12}$.

The convergence behavior of a uniform h-refinement was analyzed for a circular plate domain which is subjected to a uniformly distributed load, considering clamped boundary conditions in analogy to the problem depicted in Fig. 27 with a radius of the circular domain of $r=10$. A

Preprint, submitted to Int. J. Numer. Meth. Engng. in 2012 
M. Ruess et al. - Weak Boundary Conditions for the FCM

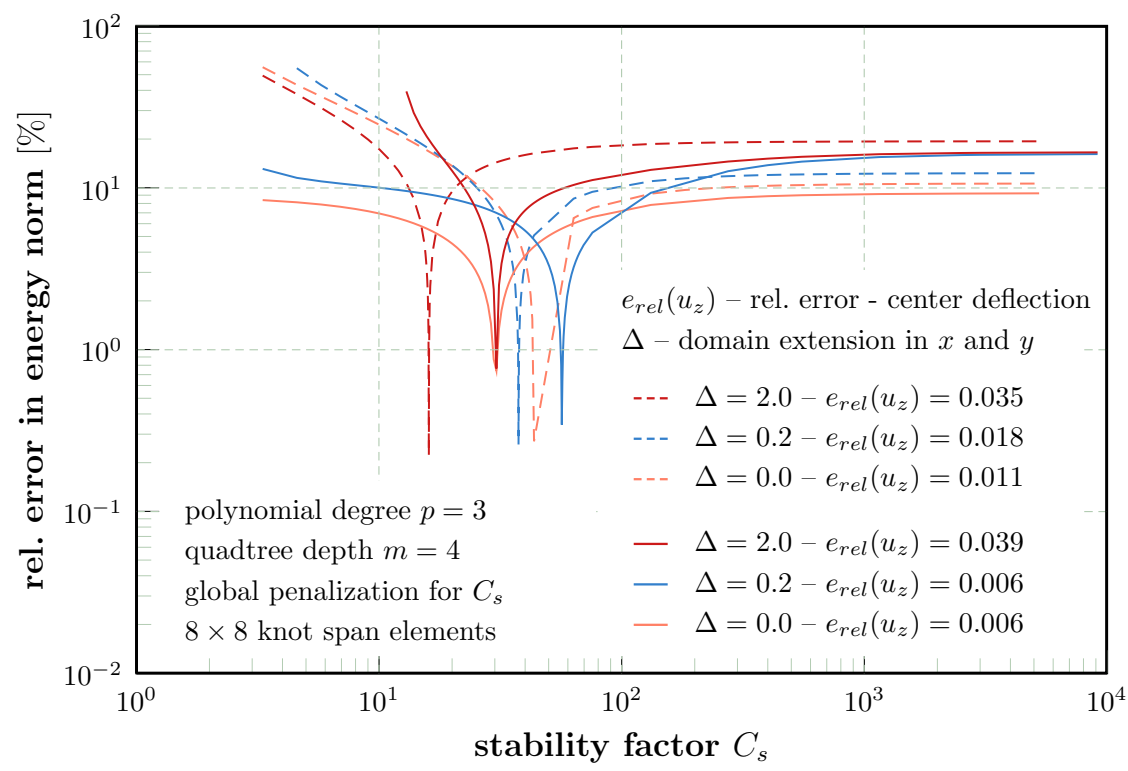

Figure 28: Stability parameter study for $C_{S}$. For $\nu=0.0$, parameter $C_{N}$ cancels out (dashed curves) whereas for $\nu=0.3$ (solid curves) the parameter $C_{N}$ was derived from an eigenvalue computation on cell level according to [20].

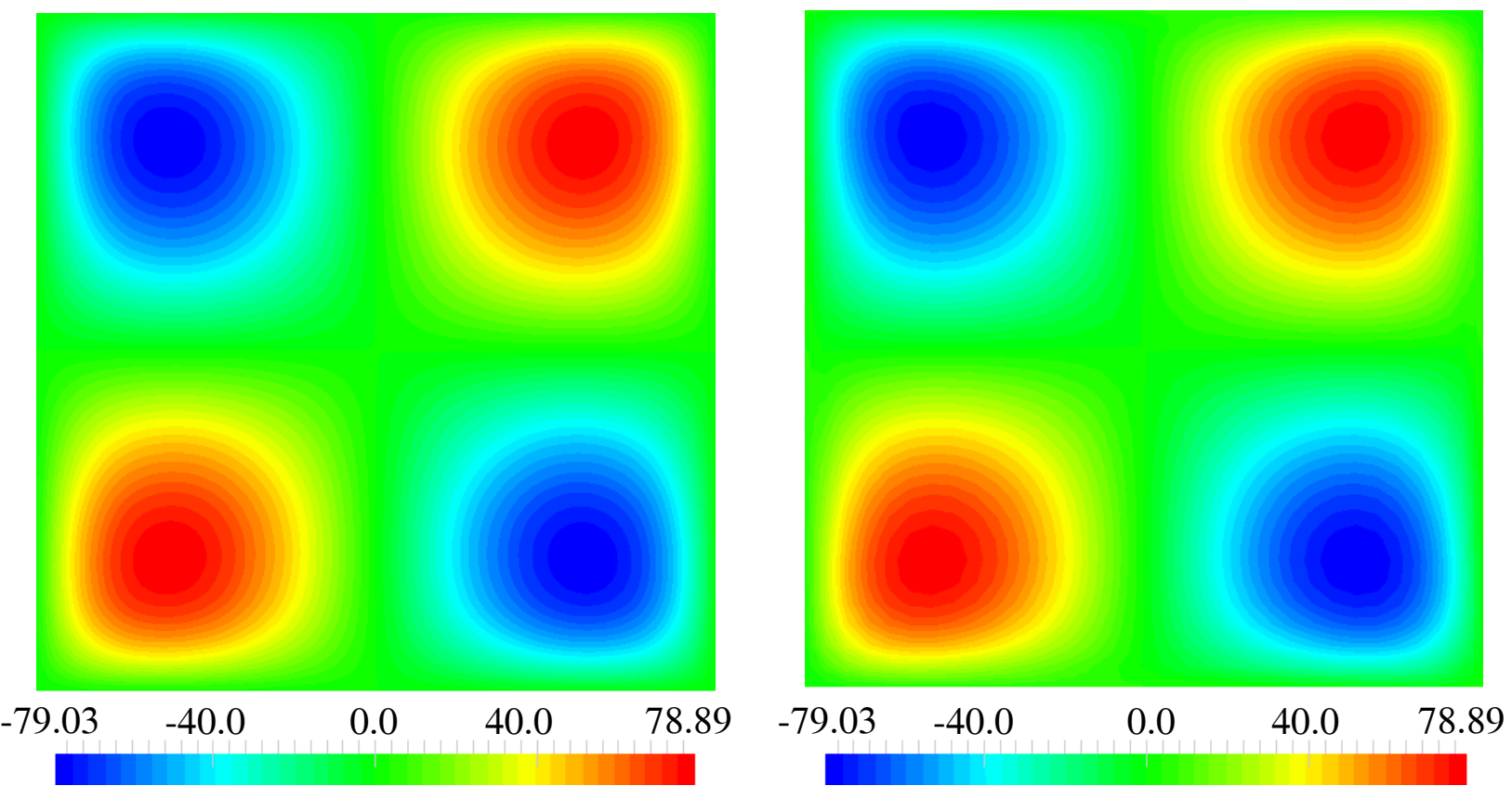

Figure 29: Qualitative comparison of the moment stress resultants referred to the mid-plane of a clamped thin plate: moment stress resultants $m_{11}-$ FCM (left), Kirchhoff-Love (right). 

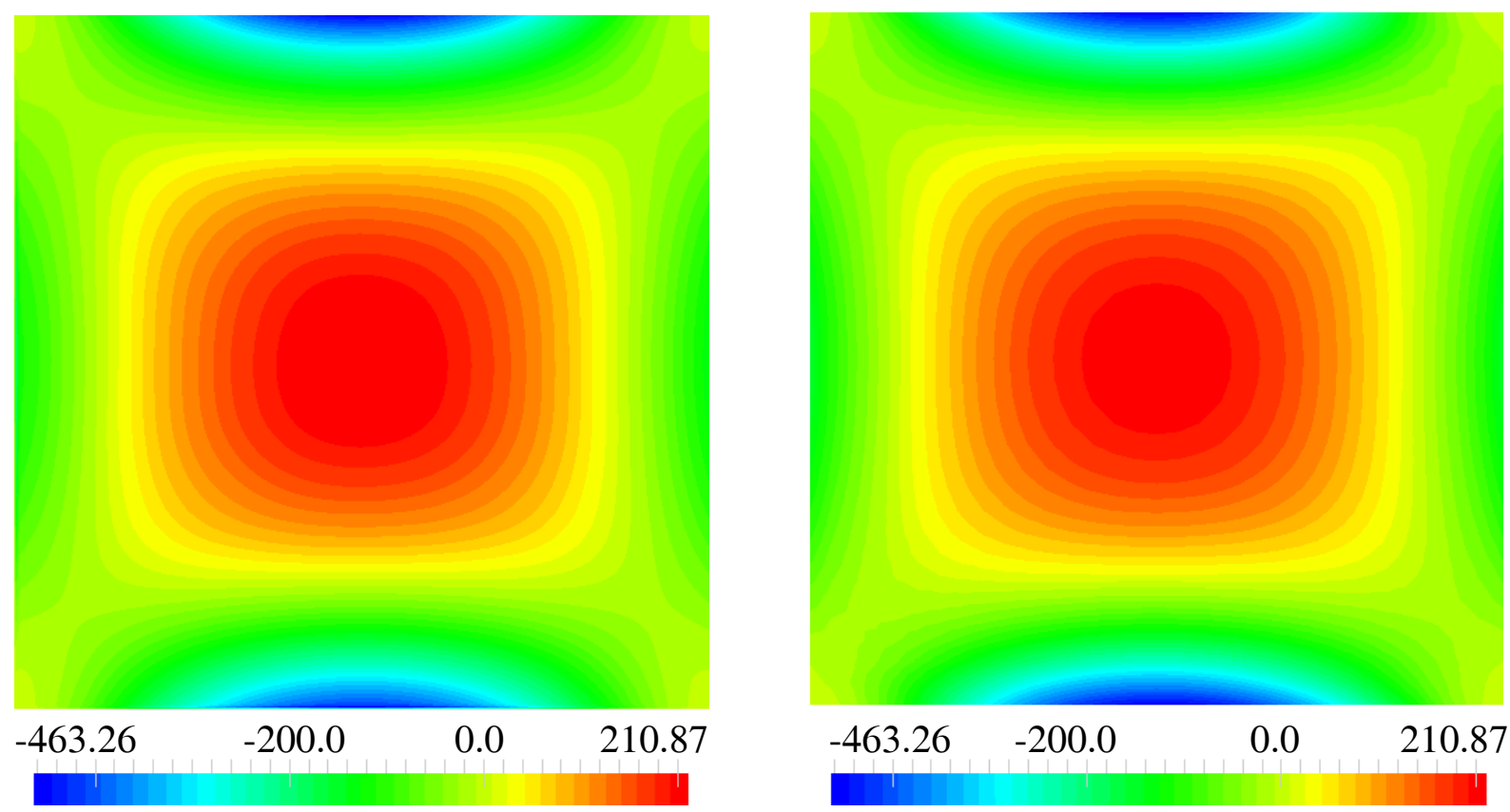

Figure 30: Qualitative comparison of the moment stress resultants referred to the mid-plane of a clamped thin plate: moment stress resultants $m_{12}-$ FCM (left), Kirchhoff-Love (right).

sufficient octree depth was chosen to ensure convergence and an overall accuracy of comparable quality. The relative error in energy norm and the relative error of the center deflection of the circular plate, both showing optimal rates, are depicted in Fig. 31.

\subsection{Crankshaft stress analysis}

We used this example of a 4-cylinder engine crankshaft (Fig. 32) to demonstrate the applicability of the proposed method for the elasticity analysis of complex structures from engineering practice. The geometric model was provided as a coarse triangle surface tessellation model. Various strategies concerning the representation of solid structures within the embedding domain $\Omega_{\alpha}$ were followed, e.g. in [42] as a BREP-model applying a tree-based radiosity algorithm to decide if domain points were inside or outside $\Omega$ or a voxel-approach $[44,49]$ that implicitly represents the model by the value of each voxel within $\Omega_{\alpha}$. The latter was applied in the following example to take advantage of a pre-computation scheme for system stiffness and load as introduced in $[55,45]$, thus significantly reducing the numerical effort required for integration and assembly of the cell properties. The crankshaft's dimensions were approximately $26 \mathrm{~cm}$ in length and $6 \mathrm{~cm}$ in diameter. The model was voxelized on the basis of recursive bisection that resulted in an octree identifying the triangle surface model. The complement of the surface model was regarded as the model's interior which was voxelized by a filling algorithm. The octants of the domain decomposing octree acted independently which allows for an efficient and synchronization-free shared memory parallelization [53].

Preprint, submitted to Int. J. Numer. Meth. Engng. in 2012 

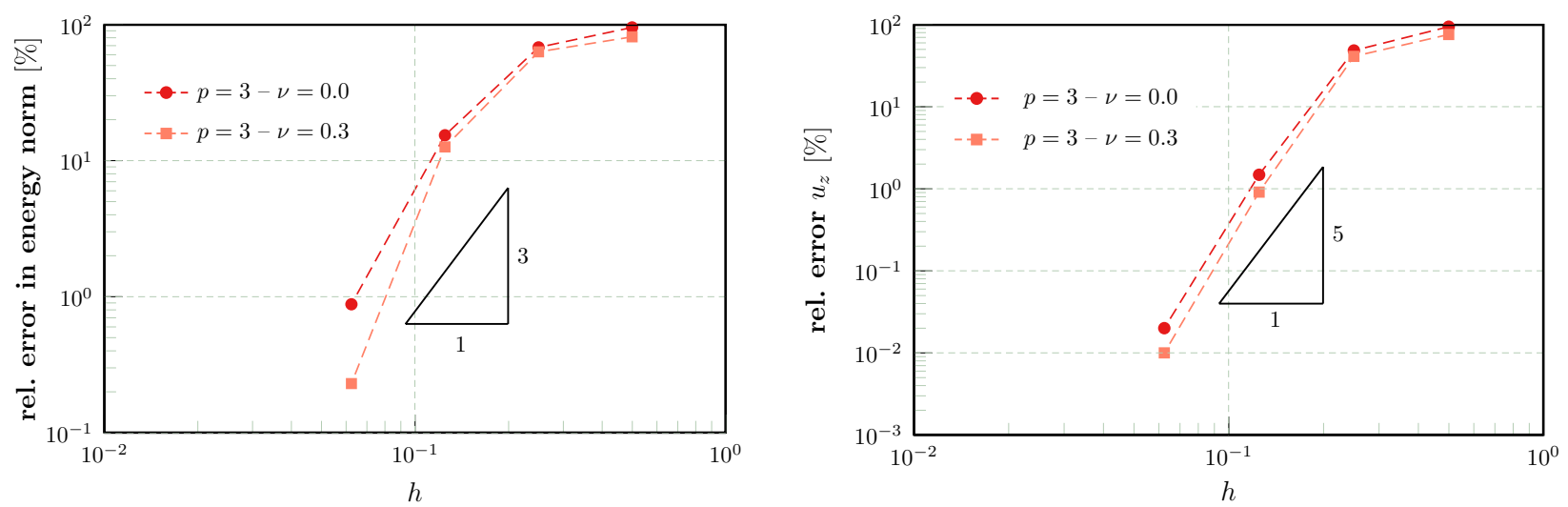

Figure 31: Relative error in energy norm (left) and relative error of the FCM center deflection (right) for uniform h-refinement.

The considered model consisted of 62.5 million voxel resulting from a $250 \times 1000 \times 250$ grid resolution which provided a sufficiently fine granularity level. Using the dimensions $0.24 \mathrm{~mm}^{3} /$ voxel the model fully resolved the crankshaft geometry including details, as shown in (Fig. 32). The time effort for the voxelization process was in the range of a few seconds on an eight core Intel(R) Xeon(R) CPU W5590 @ 3.33GHz processor.

Inside $\Omega$ a Young's modulus of $E_{\Omega}=210 G P a$ and a Poisson ratio of $\nu_{\Omega}=0.3$ were chosen. The fictitious extension domain was penalized with an $\alpha=1 . e-12$.

The considered load case included pressure loads on all four crank pins in different directions resulting from a cylinder burst and provoking a torsional load case. The crankshaft was fixed along the crank journal at the two outer journal positions (cf. displacement field Fig. 33). The weak boundary conditions were applied to the STL-surface mesh of the corresponding crank journal locations.
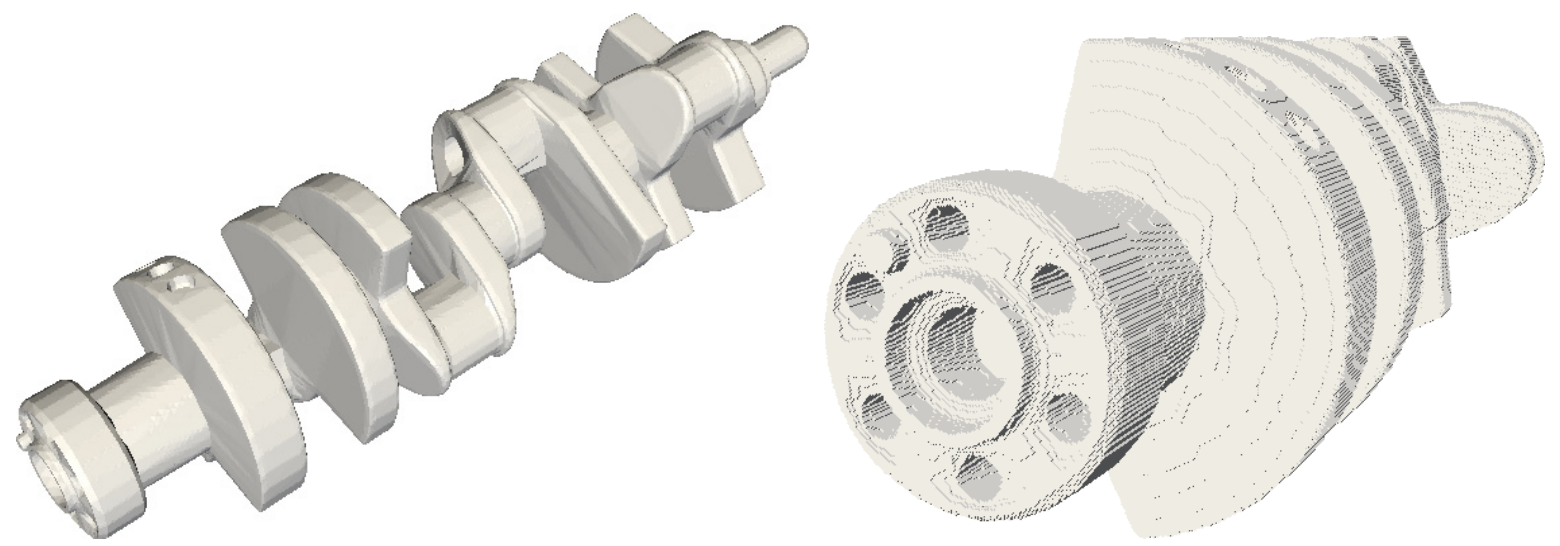

Figure 32: Crankshaft - surface model and voxel model (62.5 million voxel).

Preprint, submitted to Int. J. Numer. Meth. Engng. in 2012 


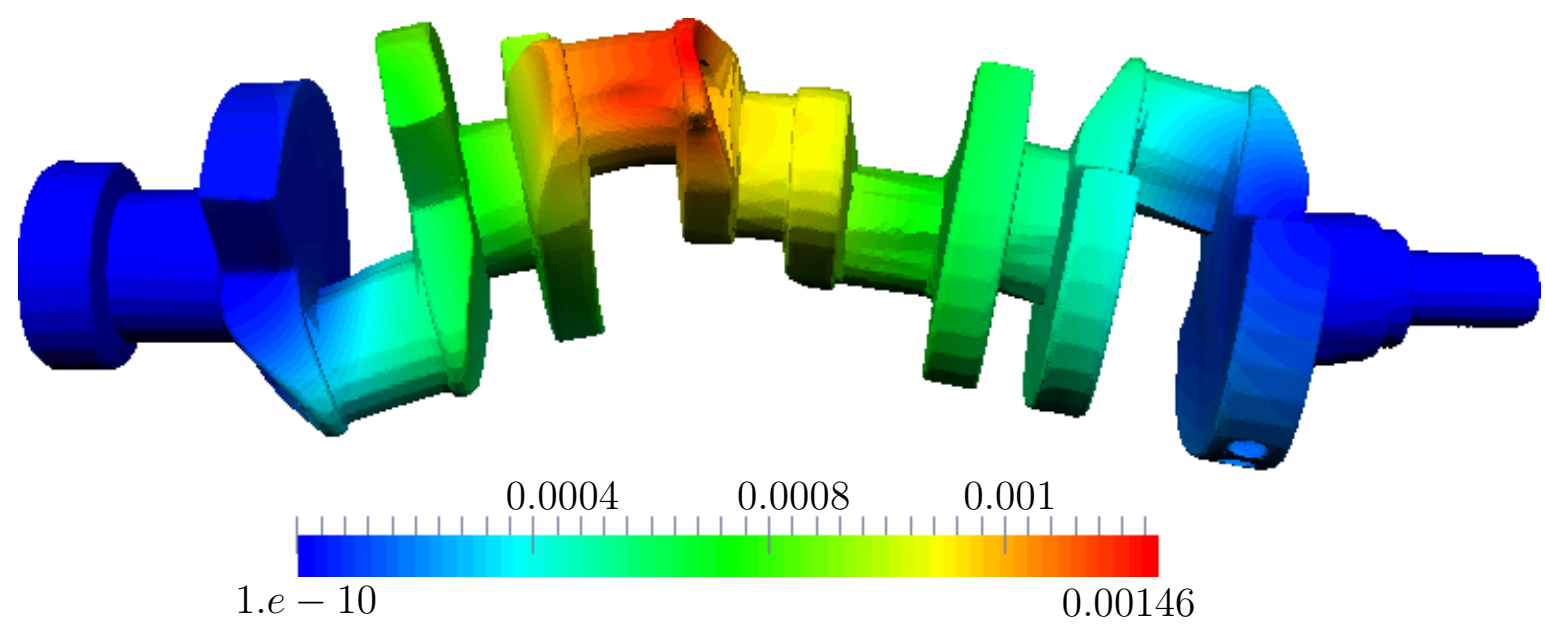

Figure 33: Crankshaft: displacement field $\left(u_{x}^{2}+u_{y}^{2}+u_{z}^{2}\right)$.

The displacement field on the deformed structure is shown in Figure 33. The weakly constraint support positions leave the flywheel and the shaft end completely undistorted and stress free. The deformation along the crank shaft corresponds the unsymmetric loading at the crankpins. Exemplarily, the shear stresses $\sigma_{12}$ are shown in Figure 34. A polynomial degree of $p=3$ was chosen for the analysis resulting in 22000 degrees of freedom.

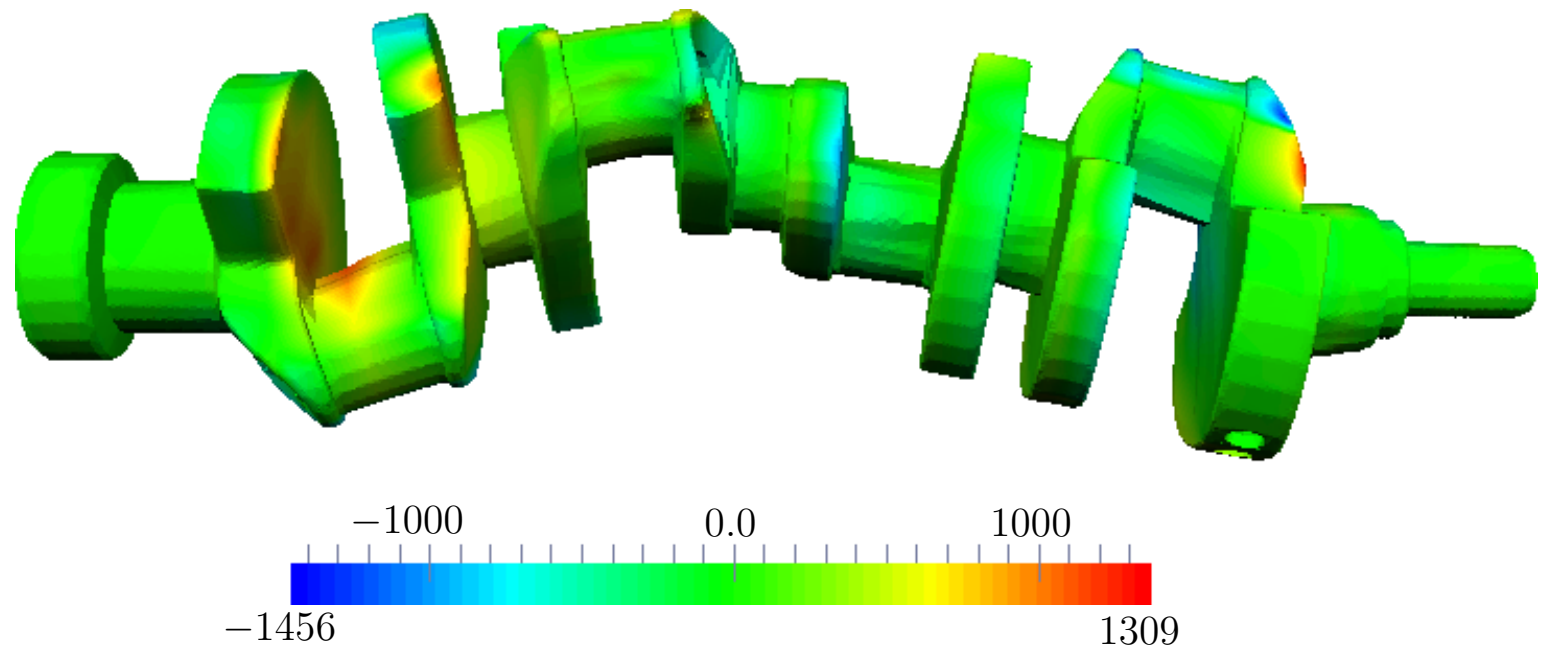

Figure 34: Crankshaft: shear stress distribution $\sigma_{12}$.

\subsection{Human femur analysis on the basis of voxel data}

The weak boundary concept was modified for heterogeneous voxel models of biomechanical simulations derived from qualitative computer tomography scans (QCT-scan data). The fol-

Preprint, submitted to Int. J. Numer. Meth. Engng. in 2012 
lowing analysis illustrates the numerical prediction of the elasticity behavior of a human femur subjected to a compression load on the femur's head.

A male femur donor was tested in-vitro with a compression test and in-silico by a high-order finite element analysis of very high quality for verification and validation purposes. The segmented femur was CT-scanned in a clinical computer tomograph in 186 slices at a resolution of $(1024 \times 1024)$ pixel per slice. We refer you to [44] for a detailed description of the testing procedure and a comparison of the test results with a p-version finite cell analysis and finite element analysis.

The QCT-resolution results in a voxel spacing of $(0.195 \mathrm{~mm} \times 0.195 \mathrm{~mm} \times 1.25 \mathrm{~mm})$. The measured radiodensity for each voxel is provided as a CT-value referred to a Hounsfield scale [14]. An additionally scanned calibration phantom allowed a linear conversion between Hounsfield Units (HU) and an equivalent bone mineral density value that served as the basis for a pointwise derivation of the bone's heterogeneous material properties, according to the model of KEYAK AND FALKINSTEIN [33] and assuming isotropic properties that are well accepted for this type of test [5], at a constant Poisson ratio of 0.3 .

The femur was fully embedded in a cell grid of 652 knot span elements (Fig. 36(a)), each covering $40 \times 40 \times 10$ voxel. In accordance with the cell-wise voxel configuration the number of sub-cells was chosen for the composed integration of the governing integrals (26) and (27), respectively, to capture fully the heterogeneity of the cortical and trabecular bone structure. The cell stiffness matrix was pre-computed for various polynomial degrees as proposed in [55] thus significantly reducing the numerical effort of the analysis. Each stiffness contribution of the $40 \times 40 \times 10$ sub-cell domains was pre-computed independently from the material's Laméparameter. A consecutive assembly loop over all finite cells scaled each pre-computed subcell contribution with the corresponding material properties and summed up the contributions to the cell stiffness matrix that incorporated point-wise all the provided heterogeneous material properties of the bone.

A compression load of $1000 N$ on top was applied at an inclination angle of $5.52^{\circ}$ corresponding to the in-vitro test (Fig. 36(c)). The load was distributed over a small circular area to mimic the pressure zone of the compression cylinder. Homogeneous boundary conditions were weakly enforced at the bone's distal face. The distribution of the Hounsfield Units and corresponding moduli of elasticity for the clamped bottom QCT-slice are shown in Figure 35. Due to the material's heterogeneity the stresses were a function of the material properties and the location factor $\alpha$ inside $\Omega_{\alpha}$ at each point.

$$
\hat{\sigma}_{\alpha}=\sigma(\alpha(\mathbf{x}), H U(\mathbf{x}))
$$

The additional HU-dependency of the stresses was considered in the extended formulation (26) and (27), respectively, and had a direct effect on the stability parameters $\tau_{S}$ and $\tau_{N}$ which varied from point to point according to (28) and (29). It is worth mentioning that the complete integration process for both the domain and the additional boundary integrals was dramatically economized by the pre-computation scheme for voxel-data which could easily be extended to the weak boundary formulation demonstrated here.

Convergence was observed with a relative error in energy norm of below $10 \%$ for a polynomial degree $p=4$ and referred to a reference value extrapolated from solutions for $p=3,4,5$. At

Preprint, submitted to Int. J. Numer. Meth. Engng. in 2012 


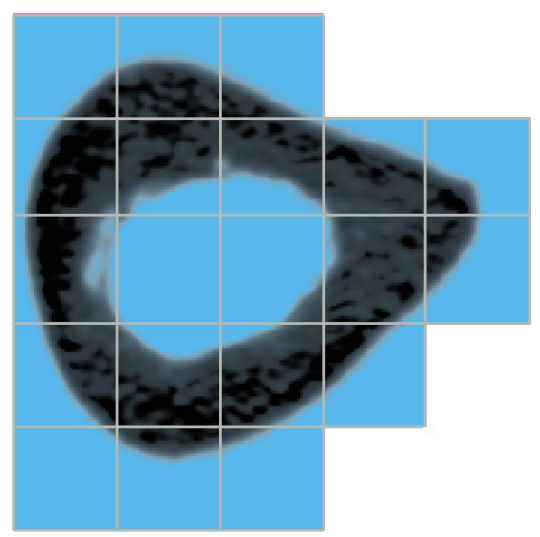

$$
\begin{array}{ll}
H U & E(H U) \\
1476- & 22438 \\
1200- & 15043 . \\
800- & 6950 . \\
400- & 576 \\
0 & -[\alpha:=] 10^{-10}
\end{array}
$$

Figure 35: Cell domain $\Omega_{\alpha}$ of the clamped bottom qCT-slice of a human femur: Hounsfield units and derived corresponding moduli of elasticity.

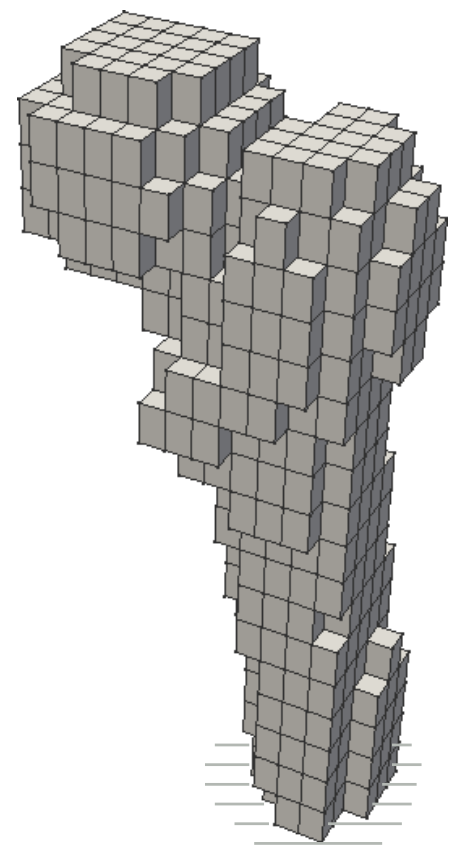

(a) knot-span cells

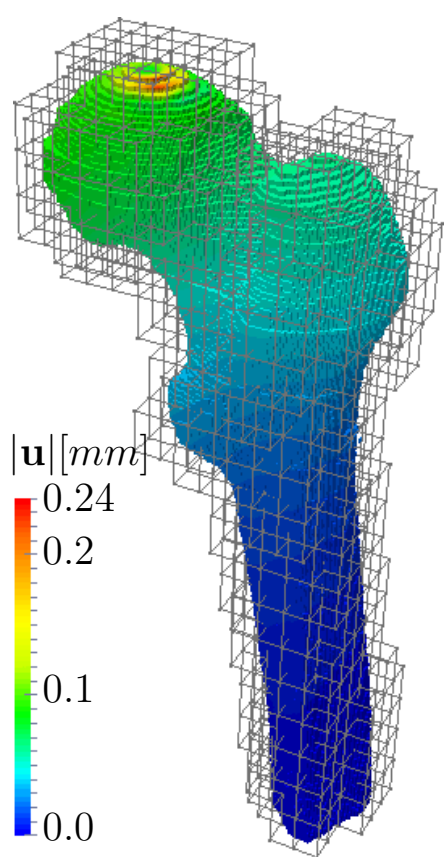

(b) displacements

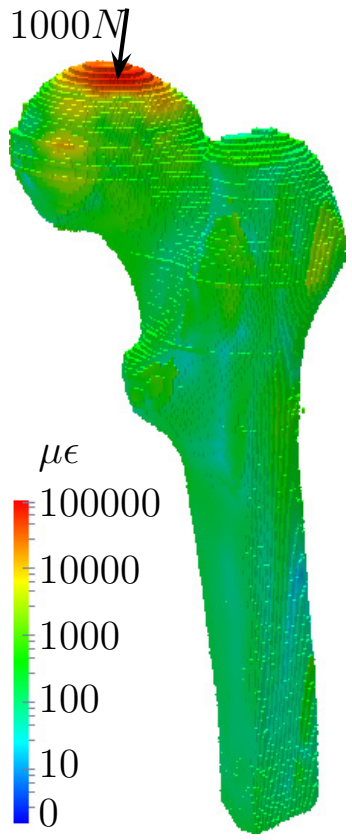

(c) von Mises equiv. strain

Figure 36: Cell model, displacement field and von Mises equivalent strain distribution of the on top loaded femur.

13 locations on the femur surface the principal strains were evaluated and compared to measurements from surface-glued strain gauges. Three readings were completely off due to an insufficient strain gauge bond and were thus discarded from the analysis results. The remaining results showed a correlation of above $98 \%$ between test results and numerical prediction. A similar result was obtained for a p-version FCM analysis and a p-FEM analysis [52, 56] on a tetrahedral 
mesh with B-spline smoothed surface (cf [44]). Both reference models confirmed the numerical prediction of the NURBS-FCM including the values for the three discarded outliers. The magnitude of the displacements and the von Mises equivalent strains are depicted in Fig. 36(b) and 36(c), respectively. It is worth mentioning that the number of degrees of freedom for the NURBS-based analysis model $(N=12,900)$ is roughly a third of the p-version FCM model $(N=35,300)$. Both FCM versions, the p-version and the NURBS-version, required one order of magnitude less degrees of freedom than the p-version FEM reference solution that has been proven with many validated results to be a very reliable, robust and accurate simulation approach $[44,52,56]$

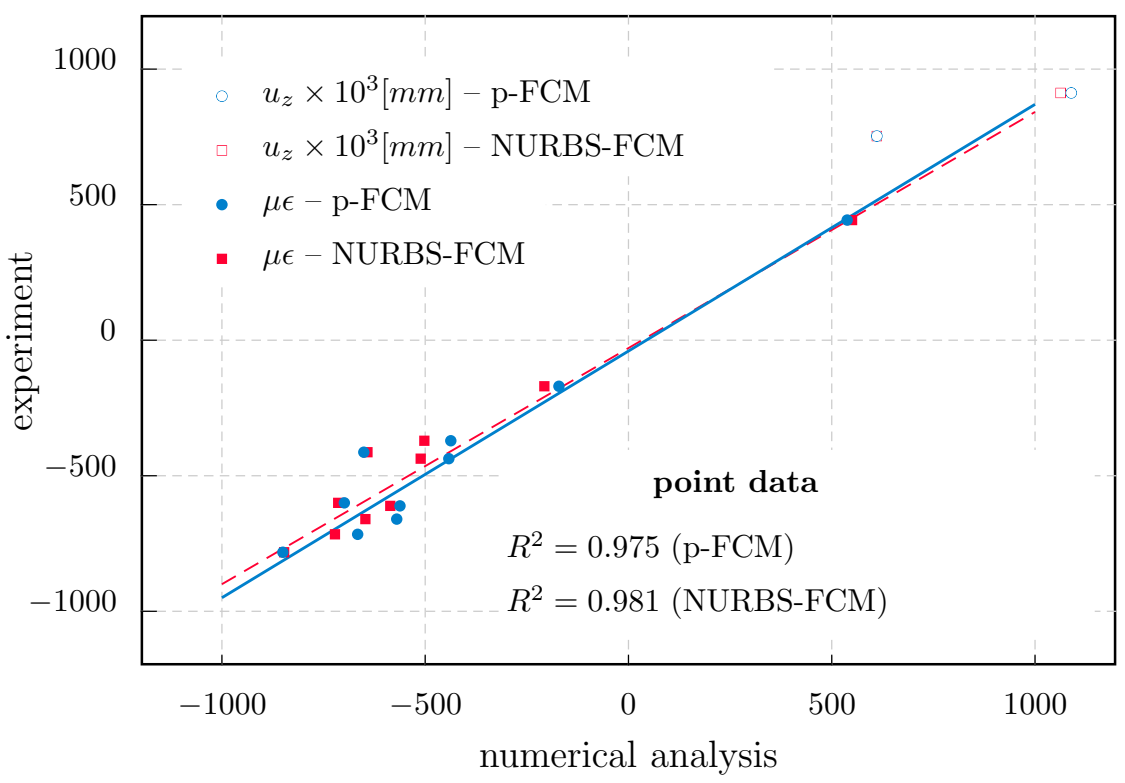

Figure 37: Validation results of a pointwise comparison.

A pointwise comparison between the measured values of the experiment and the numerical prediction is depicted in Fig. 37. The results in red describe the correlation of the strains (filled dots) and vertical displacements (outline dots) of the NURBS-based finite cell analysis versus the experimental data. A reference solution that was obtained from a finite cell analysis based on a high-order Legendre Ansatz space is shown in blue. Despite a very high correlation between the two analysis results some points deviated marginally which can be attributed to slightly different analysis models due to differences in the implementations of the two FCM-variants.

\subsection{Isogeometric analysis of a trimmed NURBS shell structure}

Finally, with this example we demonstrate the value of our developments in the context of isogeometric analysis of trimmed geometries. The combination of the fictitious domain idea and the weak enforcement of boundary conditions applied to NURBS structures as shown in the 
following has proved to be well qualified to overcome the problem of trimmed geometries in isogeometric analysis. A NURBS shell structure and corresponding material properties modeled as a volumetric analysis model is shown in Figure 38. Dirichlet boundary conditions were applied to the outer rim of the shell. The inner rim was assumed traction free. The model was subjected to a self-weight loading.
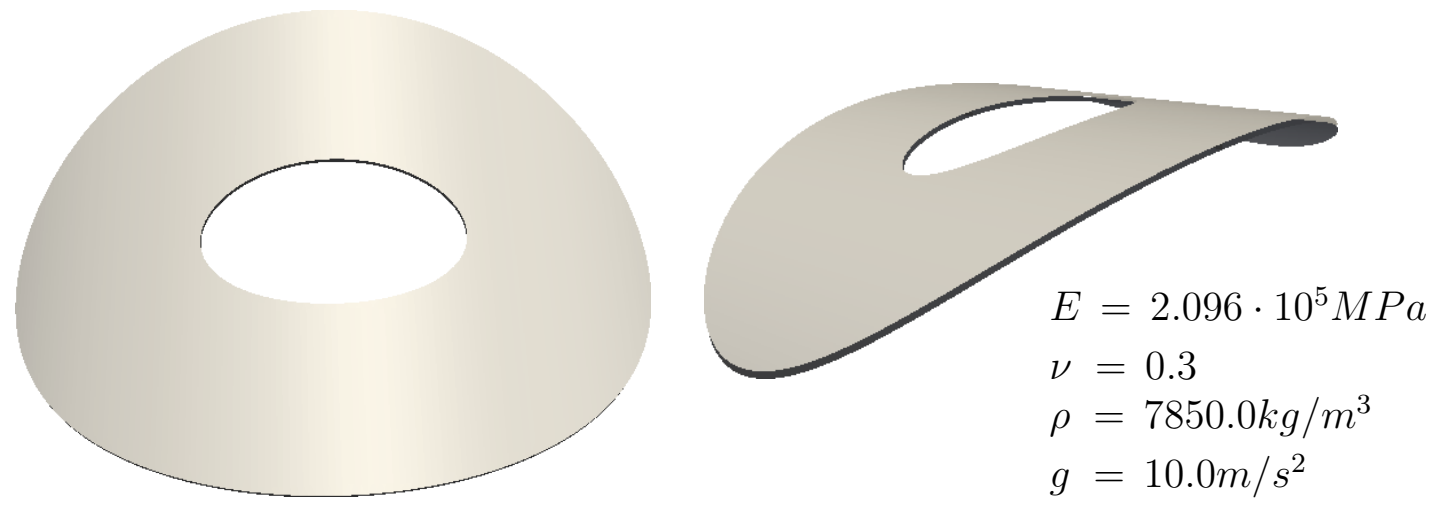

Figure 38: Trimmed NURBS geometry of a volumetric shell model.

The geometry of the shell structure was based on a NURBS patch trimmed by two NURBStrimming curves. Due to the symmetry of the structure, only one half was considered in the modelling and analysis process. The complete modelling chain is presented in Fig. 39. Both,

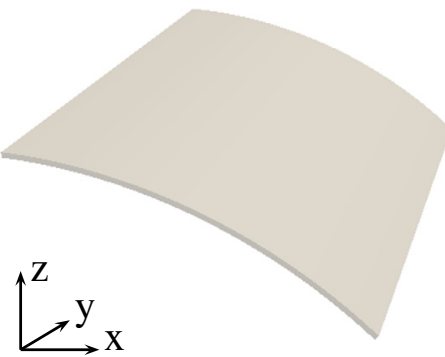

(a) initial patch

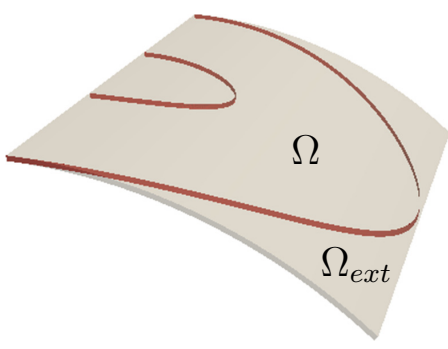

(b) trimming curves

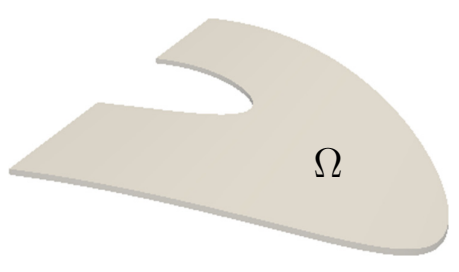

(c) final trimmed geometry

Figure 39: NURBS modelling chain.

the $y$-extension and the curvature radius of the shell structure (Fig. 39(a)) were $200 \mathrm{~mm}$. The apex angle of the curved model was $40^{\circ}$, the shell thickness was $2 \mathrm{~mm}$. Both of the trimming surfaces resulted from an intersection of elliptic cylinders with the NURBS patch. A detailed description of a geometric modelling process based on boolean CSG (constructive solid geometry) operations can be found in [42]. The outer rim surface was meshed with plane triangles for the enforcement of weak boundary conditions, exploiting the composed integration scheme of section 3.3. Symmetric boundary conditions $u_{x}=0$ were applied along the symmetry line $y$ at $x=0$. 
symmetry bc: $u_{x}=0$

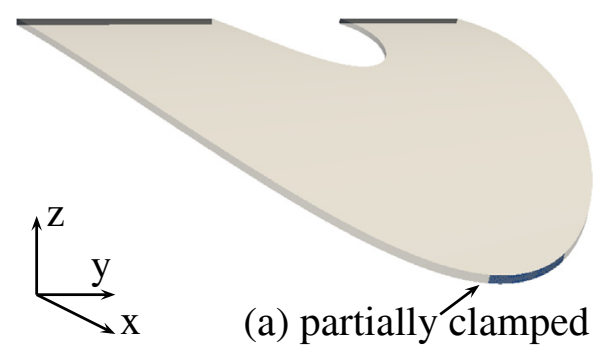

symmetry bc: $u_{x}=0$

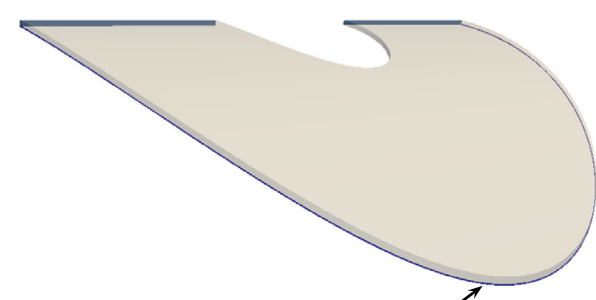

(b) simple support

Figure 40: Two variants of essential boundary conditions.

In the isogeometric analysis of the structure the trimmed domains were treated as an extension domain within a finite cell context and penalized in the stresses and forces with a value $\alpha=$ 1.e -12 . Instead of an embedding simulation domain on a Cartesian grid, this model exploited the in-plane smoothness and exactness of a NURBS geometry as provided by a NURBS modeler [23]. Two models with different types of boundary conditions along the outer rim were analysed. Homogeneous boundary conditions over a part of the rim surface simulated a clamped structure for the first model while for the second model a line boundary along the complete outer rim was chosen to represent a simple support (Fig. 40).

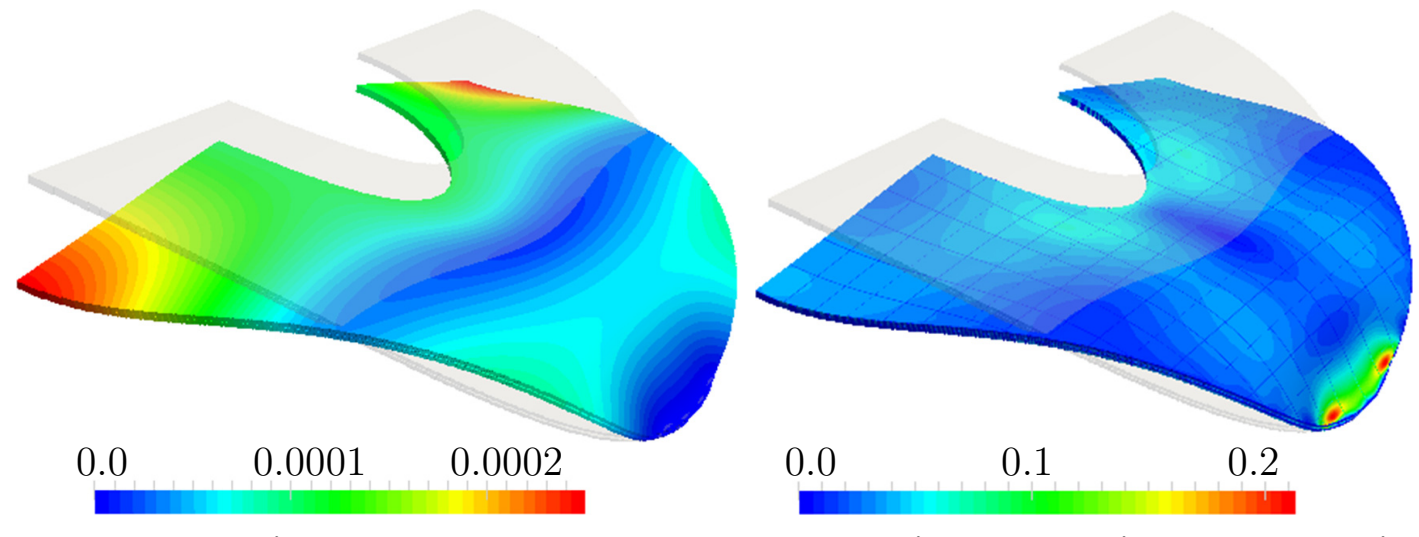

(a) displacements

(b) von Mises stress with knot span grid

Figure 41: Analysis results of the partially clamped shell on the deformed model.

The simulation model consisted of $16 \times 16 \times 1$ knot-span cells with a polynomial degree of $p=5$ in the shell plane and a cubic degree over the shell thickness. A reference solution was found for both models from a p-version FEM analysis with 840 hexahedral elements and strongly enforced essential boundary conditions. Both models, the simply supported shell and the clamped shell, converged in the energy norm for uniform p-refinement with both analysis methods and showed an equivalent displacement and stress behavior. A relative error in energy norm of $\approx 7 \%$ was found for the simply supported shell with respect to the p-FEM reference 
value that was extrapolated from successive refinement steps. The same model with partially clamped boundary conditions resulted in a relative error in the energy norm of $\approx 12 \%$. The higher error level was due to the given constraint boundary surface domain of the isogeometric model that did not exactly coincide with the constraint area of the p-FEM model depending on the nodes, edges and faces of the boundary fitted elements. However, a relative error of the maximum deflection of this model was in an acceptable range of $\approx 4 \%$.

The displacement field on the deformed structure and the von Mises stress distribution with the knot-span cell grid are depicted in Fig. 41. A membrane effect can be observed in the bulb of the lower part of the shell structure which results from the clamped surface boundary, whereas the upper part is more bending dominated as a consequence of the unconstraint boundaries. The high symmetry in the results is an indicator of the accuracy level of the analysis. The symmetric stress concentrations at the clamped rim underline this observation.

The analysis results of the simply supported system are depicted in Fig. 42. Again the principal deformation behavior is shown on the deformed structure. The steep gradient in parts of both the displacements and the von Mises stresses along the curved boundary reveal a correct deformation according to the line constraints. The membrane effects dominate the overall elasticity behavior since free deformation of the structure is fully suppressed. Typical local stress concentrations indicate bending around the elliptical opening as expected.

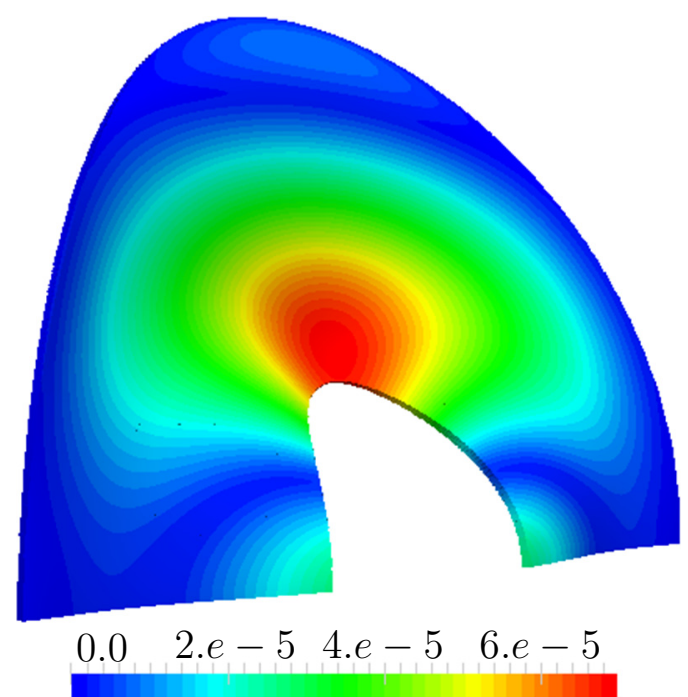

(a) displacements

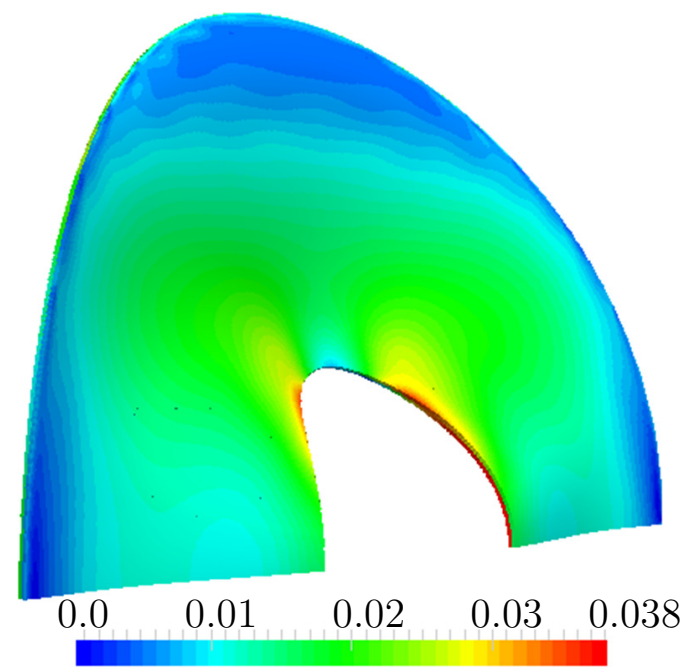

(b) von Mises stress

Figure 42: Analysis results of the simply supported shell on the deformed model.

\section{Summary and conclusions}

In this article we addressed one of the central issues of fictitious and immersed boundary methods: the enforcement of essential boundary conditions. Following the idea of Nitsche's paper 
on a weak enforcement of boundary conditions for the Poisson problem [38], we consistently extended the formulation of the principle of virtual work for the analysis of elasticity problems on the basis of the finite cell method, which is a fictitious domain approach of higher approximation order. Using the weak boundary condition formulation we essentially increased the range of applicability of the finite cell method by enhancing the method's flexibility with regard to an arbitrary embedding of the analysis structure in a fictitious simulation domain. In a principle study on the influence of the fictitious extension domain of cut cells and the weak boundary condition formulation on the conditioning of the problem we revealed some basic characteristics of the numerical simulation model, which essentially influenced the process of stabilization of the proposed formulation. We further presented a substantial decrease in the numerical integration effort required on the cell level by a rearranging the integration sequence of the composed integration concept. This simple concept is also applicable to future developments of adaptive integration within the framework of the finite cell method. Furthermore, we proposed a composed integration scheme for triangulated surface boundaries to further support a modelling process that is independent of the finite cell discretization.

The performance of the proposed method was demonstrated using several benchmark tests to illustrate its stability and accuracy, and to isolate the parameters that had the most influence on the weak enforcement of the essential boundary conditions. It was found that the overall performance of the method was primarily dominated by the integration error in the embedded solution domain. Provided there is a sufficiently small integration error in the governing domain integrals the extended formulation easily provides optimal convergence rates in h- and p-refinement. Various studies on the choice of the required stabilization values of the weak boundary condition formulation show relatively benevolent behavior with regard to the stability of the computation and the error introduced to the solution domain. The number of cut boundary cells and their share in the physical and fictitious extension domain control the need for stabilization and often justify the local treatment of stabilization that considers the specific domain properties rather than a global approach that covers each cut boundary cell identically.

A comparison of the method with a standard finite element formulation for thin plates proved the method's competitiveness in terms of solution quality already at moderate polynomial degrees and reduced modelling effort. Using a 3D model of a crankshaft we demonstrated the method's applicability to problems from engineering practice. In combination with a voxelization process on the basis of recursive bisection the complexity of the numerical modelling process was dramatically reduced to a fully automated simulation pipeline providing reliable results at various levels of accuracy, controlled by the voxel model, the cell configuration and the applied approximation order with essential boundary conditions treated independently of the embedding cell grid. Extending the method to heterogeneous voxel models derived from CT-data we illustrated a simple strategy to enforce the constraints on voxel level weakly. Verification and validation of the simulation results of an in-vitro tested human femur demonstrated an accuracy in the top level range of the available analysis software for biomechanics. Finally, the problem of trimmed geometries in isogeometric analysis was addressed using a shell-like structure. We showed that the NURBS-version of the finite cell method in combination with the proposed extension for the weak enforcement of essential boundary conditions is a practicable strategy to overcome this limitation. 
M. Ruess et al. - Weak Boundary Conditions for the FCM

Acknowledgments The authors would like to thank Prof. Z. Yosibash and his group from the Ben-Gurion University of the Negev, Israel, for providing the femur model data and the corresponding experimental results. The last author would also like to thank the German Research Foundation, which has partially supported this research under Grant No Ra624/15-2.

\section{References}

[1] I. Akkerman, Y. Bazilevs, V. M. Calo, T. J. R. Hughes, and S. Hulshoff. The role of continuity in residual-based variational multiscale modeling of turbulence. Computational Mechanics, 41:371-378, 2008.

[2] I. Babuška. The Finite Element Method with Lagrangian Multipliers. Numerische Mathematik, 20:179-192, 1973.

[3] I. Babuška. The Finite Element Method with penalty. Mathematics of Computation, 27(122):221-228, 1973.

[4] I. Babuška, B. A. Szabo, and I. N. Katz. The $p$-Version of the Finite Element Method. SIAM Journal on Numerical Analysis, 18:515-545, 1981.

[5] V. Baca, Z. Horak, P. Mikulenka, and V. Dzupa. Comparison of an inhomogeneous orthotropic and isotropic material models used for fe analyses. Medical Engineering \& Physics, 30:924-930, 2008.

[6] K.J. Bathe. Finite element procedures. Prentice Hall, 1996.

[7] Y. Bazilevs, L. Beirão da Veiga, J. A. Cottrell, T. J. R. Hughes, and G. Sangalli. Isogeometric analysis: approximation, stability and error estimates for h-refined meshes. Mathematical Models and Methods in Applied Sciences, 16(7):1031-1090, 2006.

[8] Y. Bazilevs, V. M. Calo, J. A. Cottrell, J.A. Evans, T. J. R. Hughes, S. Lipton, M.A. Scott, and T.W. Sederbergc. Isogeometric analysis using t-splines. Computer Methods in Applied Mechanics and Engineering, 199:229-263, 2010.

[9] Y. Bazilevs, V. M. Calo, Y. Zhang, and T. J. R. Hughes. Isogeometric fluid-structure interaction analysis with applications to arterial blood flow. Computational Mechanics, 38:310 $322,2006$.

[10] Y. Bazilevs and T. J. R. Hughes. Weak imposition of Dirichlet boundary conditions in fluid mechanics. Computers \& Fluids, 36:12-26, 2007.

[11] Y. Bazilevs, C. Michler, V.M. Calo, and T. J. R. Hughes. Weak dirichlet boundary conditions for wall-bounded turbulent flows. Computer Methods in Applied Mechanics and Engineering, 196(49-52):4853-4862, 2007.

Preprint, submitted to Int. J. Numer. Meth. Engng. in 2012 
M. Ruess et al. - Weak Boundary Conditions for the FCM

[12] Y. Bazilevs, C. Michler, V.M. Calo, and T. J. R. Hughes. Isogeometric variational multiscale modeling of wall-bounded turbulent flows with weakly-enforced boundary conditions on un-stretched meshes. Computer Methods in Applied Mechanics and Engineering, 199 (1316):780-790, 2010.

[13] S. Bindick, M. Stiebler, and M. Krafczyk. Fast kd-tree based hierarchical radiosity for radiative heat transport problems. International Journal for Numerical Methods in Engineering, accepted for publication, 2011.

[14] R.A. Brooks. A quantitative theory of the hounsfield unit and its application to dual energy scanning. Journal of Computer Assisted Tomography, 1(4):487-493, 1977.

[15] E. Burman and P. Hansbo. Fictitious domain finite element methods using cut elements: Ii. a stabilized Nitsche method. Applied Numerical Mathematics, In Press, Corrected Proof, 2011.

[16] J. A. Cottrell, T. J. R. Hughes, and Y. Bazilevs. Isogeometric analysis: Towards Integration of CAD and FEM. John Wiley \& Sons, 2009.

[17] J. A. Cottrell, T. J. R. Hughes, and A. Reali. Studies of refinement and continuity in isogeometric structural analysis. Computer Methods in Applied Mechanics and Engineering, 196:4160-4183, 2007.

[18] J. A. Cottrell, A. Reali, Y. Bazilevs, and T. J. R. Hughes. Isogeometric analysis of structural vibrations. Computer Methods in Applied Mechanics and Engineering, 195:5257-5296, 2006.

[19] A. Düster, J. Parvizian, Z. Yang, and E. Rank. The finite cell method for three-dimensional problems of solid mechanics. Computer Methods in Applied Mechanics and Engineering, 197:3768-3782, 2008.

[20] A. Embar, J. Dolbow, and Isaac Harari. Imposing dirichlet boundary conditions with nitsche's method and spline-based finite elements. Int. J. Numer. Meth. Engng, 83(7):877$898,2010$.

[21] C.A. Felippa. Introduction to finite element methods. University of Colorado at Boulder. http://www.colorado.edu/engineering/cas/courses.d/IFEM.d/, 2011.

[22] S. Fernández-Méndez and A. Huerta. Imposing essential boundary conditions in mesh-free methods. Comput. Methods Appl. Mech. Engrg, 193:1257-1275, 2004.

[23] flexiCAD. Rhinoceros - NURBS modeling for Windows. http://www.de.rhino3d.com/, 2011.

[24] A. Gerstenberger and W. Wall. An eXtended Finite Element Method / Lagrange Multiplier based approach for fluid-structure interaction. Computer Methods in Applied Mechanics and Engineering, 197:1699-1714, 2008.

Preprint, submitted to Int. J. Numer. Meth. Engng. in 2012 
M. Ruess et al. - Weak Boundary Conditions for the FCM

[25] M. Griebel and M.A. Schweitzer. A particle-partition of unity method. Part V: Boundary conditions. Springer-Verlag, Berlin, 2002.

[26] A. Hansbo and P. Hansbo. An unfitted finite element method, based on Nitsche's method, for elliptic interface problems. Comput. Methods Appl. Mech. Engrg, 191:537-552, 2002.

[27] P. Hansbo. Nitsche's method for interface problems in computational mechanics. GAMM Mitteilungen, 28/2:183-206, 2005.

[28] P. Hansbo and M.G. Larson. Discontinuous Galerkin Methods for Incompressible and Nearly Incompressible Elasticity by Nitsche's Method. Comput. Methods Appl. Mech. Engrg, 191(17-18):1895-1908, 2000.

[29] Peter Hansbo, Carlo Lovadina, Ilaria Perugia, and Giancarlo Sangalli. A lagrange multiplier method for the finite element solution of elliptic interface problems using non-matching meshes. Numer. Math., 100(1):91-115, 2005.

[30] K. Höllig. Finite Element Methods with B-Splines. Society for Industrial and Applied Mathematics, Philadelphia, 2003.

[31] T. J. R. Hughes, J. A. Cottrell, and Y. Bazilevs. Isogeometric analysis: CAD, finite elements, NURBS, exact geometry and mesh refinement. Computer Methods in Applied Mechanics and Engineering, 194:4135-4195, 2005.

[32] M. Juntunen and R. Stenberg. Nitsche's method for general boundary conditions. Mathematics of Computation, 78(267):1353-1374, 2009.

[33] J.H. Keyak and Y. Falkinstein. Comparison of in situ and in vitro CT-scan-based finite element model predictions of proximal femoral fracture load. J. Med. Eng. Phys., 25:781$787,2003$.

[34] R. Löhner, J.R. Cebral, F.F. Camelli, J.D. Baum, E.L. Mestreau, and O.A. Soto. Adaptive embedded/immersed unstructured grid techniques. Archives Of Computational Methods In Engineering, 14:279-301, 2007.

[35] R. Mittal and G. Iaccarino. Immersed Boundary Method. Annual Review Fluid Mechanics, 37:239-260, 2005.

[36] P. Neittaanmäki and D. Tiba. An embedding of domains approach in free boundary problems and optimal design. SIAM Journal on Control and Optimization, 33(5):1587-1602, 1995.

[37] A. Niggl, E. Rank, R.-P. Mundani, and H.-J. Bungartz. Organizing a p-version finite element computation by an octree-based hierarchy. In P. Díez and N.-E. Wiberg, editors, Proceedings of the Second International Conference on Adaptive Modeling and Simulation, pages 26-35, Barcelona, Spain, 2005.

Preprint, submitted to Int. J. Numer. Meth. Engng. in 2012 
M. Ruess et al. - Weak Boundary Conditions for the FCM

[38] J. Nitsche. Über ein Variationsprinzip zur Lösung von Dirichlet-Problemen bei Verwendung von Teilräumen, die keinen Randbedingungen unterworfen sind. Abhandlung aus dem Mathematischen Seminar der Universität Hamburg, 36:9-15, 1970.

[39] J. Parvizian, A. Düster, and E. Rank. Finite cell method - h- and p-extension for embedded domain problems in solid mechanics. Computational Mechanics, 41:121-133, 2007.

[40] C. Peskin. The Immersed Boundary Method. Acta Numerica, 11:1-39, 2002.

[41] L. Piegl and W. Tiller. The Nurbs Book. Springer-Verlag, 2. edition, 1997.

[42] E. Rank, M. Ruess, S. Kollmannsberger, D. Schillinger, and A. Düster. Geometric modeling, Isogeometric Analysis and the Finite Cell Method. Computer Methods in Applied Mechanics and Engineering, DOI: 10.1016/j.cma.2012.05.022, 2012.

[43] M. Ruess and JP. Pahl. An extended qr-solver for large profiled matrices. International Journal for Numerical Methods in Engineering, 79:1419-1442, 2009.

[44] M. Ruess, D. Tal, N. Trabelsi, Z. Yosibash, and E. Rank. The Finite Cell Method for bone simulations: Verifcation and validation. Biomechanics and Modeling in Mechanobiology, 11(3):425-437, 2012.

[45] M. Ruess, V. Varduhn, Z. Yosibash, and E. Rank. A parallel high-order fictitious domain approach for biomechanical applications. In ISPDC Conference, Munich, Germany, IEEESeries, 2012.

[46] D. Schillinger, L. Dede, M.A. Scott, J.A. Evans, M.J. borden, E. Rank, and T.J.R. Hughes. An Isogeometric design-through-analysis methodology based on adaptive hierarchical refinement of NURBS, immersed boundary methods, and T-spline CAD surfaces. Computer Methods in Applied Mechanics and Engineering, 2012.

[47] D. Schillinger, A. Düster, and E. Rank. The $h p$ - $d$ adaptive Finite Cell Method for geometrically nonlinear problems of solid mechanics. International Journal for Numerical Methods in Engineering, 89(9):1171-1202, 2012.

[48] D. Schillinger and E. Rank. An unfitted $h p$ adaptive finite element method based on hierarchical B-splines for interface problems of complex geometry. Computer Methods in Applied Mechanics and Engineering, 200(47-48):3358-3380, 2011.

[49] D. Schillinger, M. Ruess, N. Zander, Y. Bazilevs, A. Düster, and E. Rank. Small and large deformation analysis with the p- and B-spline versions of the Finite Cell Method. Computational Mechanics, 50(4):445-478, 2012.

[50] R. Schmidt, R. Wüchner, and K.U. Bletzinger. Isogeometric Analysis of trimmed NURBS geometries. Computer Methods in Applied Mechanics and Engineering, 241-244:93-111, 2012 . 
M. Ruess et al. - Weak Boundary Conditions for the FCM

[51] B.A. Szabó, A. Düster, and E. Rank. The p-version of the Finite Element Method. In E. Stein, R. de Borst, and T. J. R. Hughes, editors, Encyclopedia of Computational Mechanics, volume 1, chapter 5, pages 119-139. John Wiley \& Sons, 2004.

[52] N. Trabelsi, Z. Yosibash, and C. Milgrom. Validation of subjectspecific automated p-fe analysis of the proximal femur. $j b, 42: 234-241,2009$.

[53] V. Varduhn, R.-P. Mundani, E. Rank. A framework for parallel numerical simulations on multi-scale geometries. Proc. of the The 11th International Symposium on Parallel and Distributed Computing - ISPDC 2012, 2012.

[54] Z. Yang, S. Kollmannsberger, A. Düster, M. Ruess, R. Burgkart, E. Garcia, and E. Rank. Non-standard bone simulation: Interactive numerical analysis by computational steering. Computing and Visualization in Science, 14(5):207-216, 2012.

[55] Z. Yang, M. Ruess, S. Kollmannsberger, A. Düster, and E. Rank. An efficient integration technique for the voxel-based Finite Cell Method. International Journal for Numerical Methods in Engineering, 91(5):457-471, 2012.

[56] Z. Yosibash, R. Padan, L. Joskowicz, and C. Milgrom. A CT-based high-order finite element analysis of the human proximal femur compared to in-vitro experiments. Journal of Biomechanical Engineering, 129:297-309, 2007.

[57] N. Zander, S. Kollmannsberger, M. Ruess, Z. Yosibash, and E. Rank. The Finite Cell Method for Linear Thermoelasticity. Computers and Mathematics with Applications, accepted, 2012.

[58] T. Zhu and S.N. Atluri. A modified collocation method and a penalty formulation for enforcing the essential boundary conditions in the element free galerkin method. Computational Mechanics, 21(3):211-222, 1998.

[59] O.C. Zienkiewicz and R.L. Taylor. The Finite Element Method - Its Basis and Fundamentals, volume 1. Butterworth-Heinemann, 6th edition, 2005.

Preprint, submitted to Int. J. Numer. Meth. Engng. in 2012 